\title{
WestVirginiaUniversity
}

THE RESEARCH REPOSITORY @ WVU

Graduate Theses, Dissertations, and Problem Reports

2012

\section{Spins in Thin Films and Nanodevices}

\author{
Phillip Tabor \\ West Virginia University
}

Follow this and additional works at: https://researchrepository.wvu.edu/etd

\section{Recommended Citation}

Tabor, Phillip, "Spins in Thin Films and Nanodevices" (2012). Graduate Theses, Dissertations, and Problem Reports. 3562.

https://researchrepository.wvu.edu/etd/3562

This Dissertation is protected by copyright and/or related rights. It has been brought to you by the The Research Repository @ WVU with permission from the rights-holder(s). You are free to use this Dissertation in any way that is permitted by the copyright and related rights legislation that applies to your use. For other uses you must obtain permission from the rights-holder(s) directly, unless additional rights are indicated by a Creative Commons license in the record and/ or on the work itself. This Dissertation has been accepted for inclusion in WVU Graduate Theses, Dissertations, and Problem Reports collection by an authorized administrator of The Research Repository @ WVU.

For more information, please contact researchrepository@mail.wvu.edu. 


\title{
Spins in Thin Films and Nanodevices
}

\author{
Phillip Tabor
}

Dissertation submitted to the Eberly College of Arts and Sciences at West Virginia University in partial fulfillment of the requirements for the degree of Doctor of Philosophy in Physics

David Lederman, Ph.D., Chair

Sergei Urazhdin, Ph.D.

Arthur Weldon, Ph.D.

Alan Bristow, Ph.D.

Peter Gannett, Ph.D.

Department of Physics

Morgantown, West Virginia

2012

Keywords:

Nanomagnetism, Topological Insulators, Exchange Bias

Copyright 2012 Phillip Tabor 


\begin{abstract}
The central theme of this work is the engineering of devices and materials that exhibit spin dependent phenomena. In particular, the spin orientation of charge carriers can play a central role in transport, especially in magnetic or other spin correlated media. Propagation of charge carriers with net spin results in a transfer of angular momentum that can excite static and dynamical states in active device elements. To utilize such phenomena in practical devices, new mew means of device characterization and optimization must be developed. To that end, we have performed experiments which elucidate some of the mechanisms underlying spin dependent transport phenomena.

We report the observation of hysteretic synchronization of point contact spin torque nanooscillators (STNOs) by a microwave magnetic field. The hysteresis was asymmetric with respect to the frequency detuning of the driving signal, and appeared in the region of a strong dependence of the oscillation frequency on the bias current. Theoretical analysis showed that hysteretic synchronization occurred when the width of the synchronization range, enhanced by the oscillator's nonlinearity, became comparable to the dissipation rate, while the observed asymmetry was a consequence of the nonlinear dependence of frequency on the bias current.

Another emergent phenomenon was a series of fractional synchronization regimes in a STNO driven by a microwave field. These regimes are characterized by rational relations between the driving frequency and the frequency of the oscillation. Analysis based on the phase model of autooscillator indicates that fractional synchronization becomes possible when the driving signal breaks the symmetry of the oscillation, while the synchronization ranges are determined by the geometry of the oscillation orbit. Measurements of fractional synchronization were utilized to obtain information about the oscillation characteristics in nanoscale systems not accessible to direct imaging techniques.

Oxidation in magnetic nanosystems can result in changes of the magnetic ordering of active layers in devices, resulting in degraded device performance. We demonstrate that magnetic multilayer nanopillars can be efficiently protected from oxidation by coating with silicon. Both the protected and the oxidized nanopillars exhibited an increase of reversal current at cryogenic temperatures. However the magnetic excitation onset current increased only in the oxidized samples. We show that oxidized nanopillars exhibit anomalous switching statistics at low temperature, providing a simple test for the quality of magnetic nanodevices.

We studied exchange bias in magnetic multilayers incorporating antiferromagnet $\mathrm{CoO}$ doped with up to 35 atomic percent of Pt. The exchange bias increased with doping in epitaxial films, but did not significantly change in polycrystalline films at the lowest measured temperature of $5 \mathrm{~K}$, and decreased at higher temperatures. We explain our results by the increased granularity of the doped antiferromagnetic films, resulting in simultaneous enhancement of the uncompensated spin density and reduction of the magnetic stability of antiferromagnetic grains.

Finally, we demonstrate the growth of $\mathrm{Bi}_{2} \mathrm{Se}_{3}$, a material known as a topological insulator (TI). The structural and electronic properties of $\mathrm{Bi}_{2} \mathrm{Se}_{3}$ films grown on $\mathrm{Al}_{2} \mathrm{O}_{3}$ (110) by molecular beam epitaxy were investigated. The epitaxial films grew in the Frank-van der Merwe mode and were c-axis oriented. They exhibited the highest crystallinity, the lowest carrier concentration, and optimal stoichiometry at a substrate temperature of $200{ }^{\circ} \mathrm{C}$ determined by the balance between surface kinetics and desorption of selenium. The crystallinity of the films improved with increasing selenium/bismuth flux ratio.
\end{abstract}




\section{Acknowledgements}

I would first like to thank Sergei Urazhdin for his guidance in my Ph.D program. Without his input and support, the work on the spin torque oscillators and exchange biased systems would not have been possible. I would also like to thank him for the instruction in the fundamental physics of semiconductors, nanoscale systems, and magnetic phenomena in general.

I thank David Lederman for his support in the growth of the $\mathrm{Bi}_{2} \mathrm{Se}_{3}$ thin films by molecular beam epitaxy. His expertise in material science and the scientific method were central to the success of that work.

Arthur Weldon also played a rather significant role in my education. Through his instruction I learned the fundamentals of the core subjects in physics. Moreover, his teaching has given me the confidence to be able to discuss science without fear of facing humiliation for having forgotten some basic facts.

I must also acknowledge significant support from the physics department. Special thanks to Sherry Puskar, Devon Cleland and Siobahn Byrne for their invaluable assistance and administrative guidance over the years. Without their support, none of the department's activities would be possible.

In addition, I thank Kineshma Munbodh, Weng Lee Lim, Joseph Rowley, Trent Johnson, Cameron Keenan, Felio Perez, and Harley Hart for their invaluable assistance in learning, trouble shooting, and using the myriad of scientific instruments required for this work. 


\section{Contents}

Acknowledgements ............................. iii

List of Figures . . . . . . . . . . . . . . . . . . . . . . . . vii

1 Introduction $\quad 1$

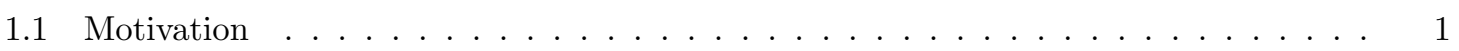

1.2 Magnetic Ordering in Materials . . . . . . . . . . . . . . . . . . . . 3

1.2.1 Exchange Bias ....................... 4

1.3 Spin Dynamics and Transport in Magnetic Systems . . . . . . . . . . . . . . . . 5

1.3.1 Magnetization Dynamics and Ferromagnetic Resonance . . . . . . . . . 5

1.3.2 Giant Magnetoresistance . . . . . . . . . . . . . . . . 10

1.3.3 Spin Transfer Torque . . . . . . . . . . . . . . . . . . . 15

1.4 Synchronization . . . . . . . . . . . . . . . . . . . . . . . . 19

1.5 Non-trivial Spin Textures: Topological Insulators . . . . . . . . . . . . . . . . . . . . 20

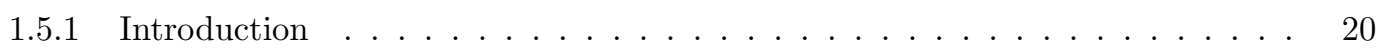

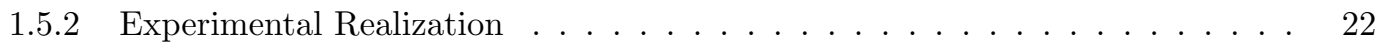

2 Experimental Techniques $r 25$

2.1 Thin Film Growth and Nanofabrication . . . . . . . . . . . . . . . . . 25

2.1.1 Molecular Beam Epitaxy _.................... 25

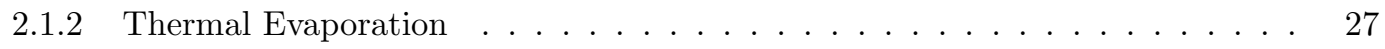

2.1.3 Magnetron Sputtering ...................... 28

2.1.4 Electron-beam Lithography . . . . . . . . . . . . . . . . . . . . . 29

2.1.5 Argon Ion Milling . . . . . . . . . . . . . . . . . . . 30 
2.2 Sample Characterization and Measurements . . . . . . . . . . . . . . . 31

2.2 .1 Atomic Force Microscopy . . . . . . . . . . . . . . . . . . . . . 31

$2.2 .2 \quad$ X-ray Diffraction . . . . . . . . . . . . . . . . . . . . . 32

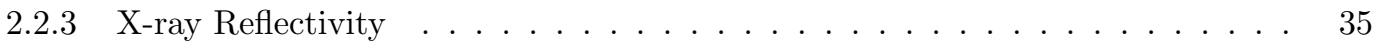

2.2.4 Reflectivity High Energy Electron Diffraction . . . . . . . . . . . . . . . 35

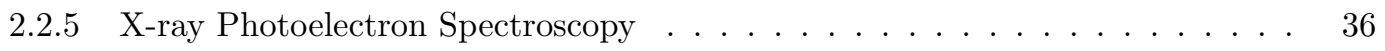

2.2 .6 The Hall Effect . . . . . . . . . . . . . . . . . . . . . . 37

2.2.7 Electronic Measurements . . . . . . . . . . . . . . . . . . . . 39

3 Synchronization of Spin Torque Nano-Oscillators 42

3.1 Device Fabrication . . . . . . . . . . . . . . . . . . . . . . . . 42

3.1 .1 Nanopillars for Current Induced Switching . . . . . . . . . . . . . . . . . 42

3.1.2 Nanopillars for Fractional Synchronization . . . . . . . . . . . . . . . . . . 43

3.1 .3 Point Contacts for Hysteresis . . . . . . . . . . . . . . . . . . 43

3.2 Microwave Stripline Calibration . . . . . . . . . . . . . . . . . . . . . . 44

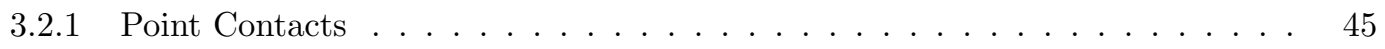

$3.2 .2 \quad$ Nanopillars . . . . . . . . . . . . . . . . . . . . . . . . . . . . . 46

3.3 Observation of Fractional Synchronization . . . . . . . . . . . . . . . . . 46

3.3.1 Introduction to Fractional Synchronization . . . . . . . . . . . . . . 46

3.3.2 Device Characterization . . . . . . . . . . . . . . . . . . . 47

3.3.3 Experimental Results and Discussion . . . . . . . . . . . . . . . . . 48

3.3.4 Dependence of Spectra on Current and Magnetic Field . . . . . . . . . . . . 50

3.3.5 Theoretical Model of Fractional Synchronization . . . . . . . . . . . . . 52

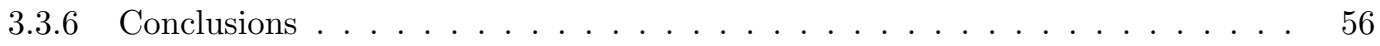

3.4 Observation of Synchronization Hysteresis . . . . . . . . . . . . . . . . . . . 56

3.4.1 Introduction to Hysteretic Synchronization $\ldots \ldots \ldots \ldots$

3.4 .2 Device Characterization . . . . . . . . . . . . . . . . . 57

3.4 .3 Experimental Results and Discussion . . . . . . . . . . . . . . . . 61

3.4 .4 Parametric Hysteresis $\ldots \ldots \ldots \ldots$. . . . . . . . . . . . . 62

3.4.5 Theoretical Model of Hysteretic Synchronization . . . . . . . . . . . . . . . 64 
3.4 .6 Conclusions . . . . . . . . . . . . . . . . . . . . . . 67

4 Magnetic Ordering in Nanoscale Systems $\quad 68$

4.1 Effect of Antiferromagnetic Ordering on Current-Induced Switching . . . . . . . 68

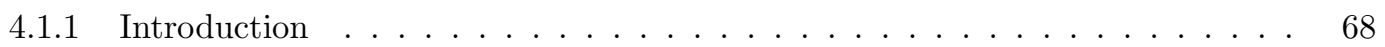

4.1.2 Experimental Results and Discussion . . . . . . . . . . . . . . . . . 69

4.1 .3 Conclusions . . . . . . . . . . . . . . . . . . . . . 73

4.2 Modification of Exchange Bias in $\mathrm{CoO}$ by Pt Doping . . . . . . . . . . . . . . . . 74

4.2 .1 Introduction $\ldots \ldots \ldots \ldots \ldots \ldots \ldots \ldots \ldots \ldots$

4.2 .2 Sample Fabrication . . . . . . . . . . . . . . . . . . . 75

4.2 .3 Experimental Results and Discussion . . . . . . . . . . . . . . . . . . . 77

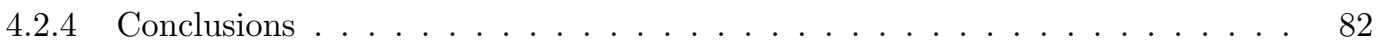

5 Engineering of Topologically Nontrivial Materials $\quad 83$

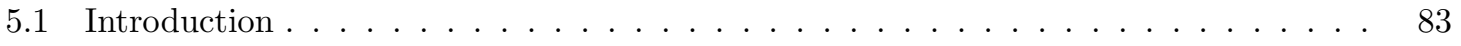

5.2 Growth of Ultra-thin $\mathrm{Bi}_{2} \mathrm{Se}_{3}$ by Molecular Beam Epitaxy . . . . . . . . . . . . . 84

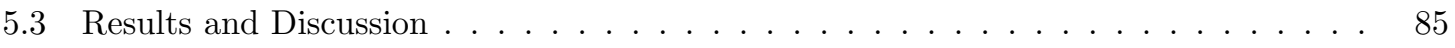

$5.4 \mathrm{Bi}_{2} \mathrm{Se}_{3}$ Flake Oxidation $\ldots \ldots \ldots \ldots \ldots \ldots \ldots \ldots \ldots$

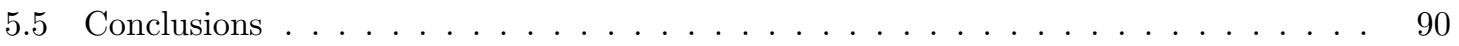




\section{List of Figures}

1 Depiction of magnetic ordering in materials. . . . . . . . . . . . . 3

2 Magnetization curves for materials with different magnetic ordering. . . . . . . . . 5

3 Schematic representation of electron transport in a multilayer structure. . . . . . . . 11

4 Equivalent circuit for the multilayers in Fig. $4 . \ldots \ldots \ldots \ldots \ldots$

$5 \quad$ Illustration of current measurement geometries. . . . . . . . . . . . . . . . 14

6 Cartoon depiction of an electron incident on a ferromagnet. . . . . . . . . . . . 15

7 Cartoon depiction of the action of the various torques on the ferromagnet. . . . . 18

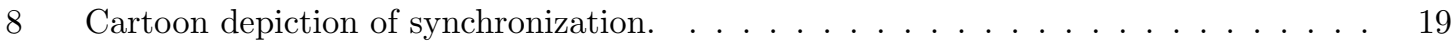

9 Representations of the quantum Hall, quantum spin Hall and topological insulator

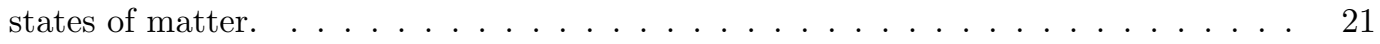

10 ARPES spectrum of a cleaved bulk crystal of $\mathrm{Bi}_{2} \mathrm{Se}_{3} \ldots \ldots \ldots \ldots \ldots \ldots$

11 Representation of a typical molecular beam epitaxy system. . . . . . . . . . . . 26

12 Cartoon depicting the DC magnetron sputtering process. . . . . . . . . . . . . 28

13 Illustration of a typical electron-beam lithography system. . . . . . . . . . . . . . 30

14 Schematics for contact and tapping modes AFM. . . . . . . . . . . . . . . 33

15 Representation of Bragg's law. . . . . . . . . . . . . . . . . . 34

16 Illustration of a typical four circle diffractometer. From reference [37]. . . . . . . . . 34

17 Schematic of a typical RHEED setup. From reference $[29] . \quad \ldots \ldots \ldots$. . . . . . . 36

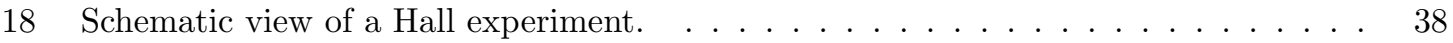

19 Schematic view of the microwave spectroscopy measurement. . . . . . . . . . . . 39

20 Depiction of the orientation of applied microwave and static fields. . . . . . . . . . 40 
21 Depiction of the van der Pauw technique. . . . . . . . . . . . . . .

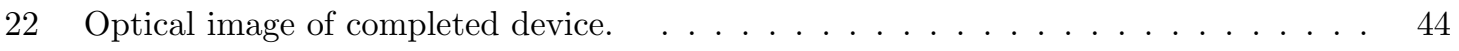

23 Scanning electron micrograph image of a $100 \times 50 \mathrm{~nm}$ pillar. . . . . . . . . . . . 44

24 Calibration of microwave field produced by microwave stripline. . . . . . . . . . . . 45

25 Device characterization for nanopillar device in the fractional synchronization exper-

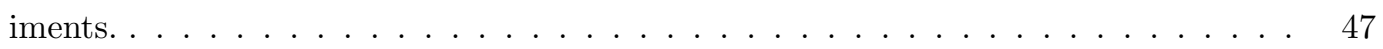

26 Microwave spectra demonstrating fractional synchronization. . . . . . . . . . . . 48

27 Oscillation properties for STNO driven in the parametric regime. . . . . . . . . . 49

28 Dependence of $\Delta f_{2}$ and $\Delta f_{5 / 2}$ synchronization regimes on microwave field. $\ldots \ldots$

29 Microwave spectra of fractional synchronization at $h_{e}=13$ Oe and $I_{0}=1.3 \mathrm{~mA}$ with

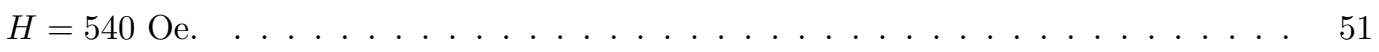

30 Dependence of fractional synchronization on direction of applied DC magnetic field. 52

31 Simulated dependence of $f_{0}^{\prime}$ on $f_{e}$, for different orientations of $\mathbf{h}_{e}$ with respect to $\mathbf{s} . \quad 55$

32 Differential resistance curve for the point contact device. . . . . . . . . . . 58

33 Characterization of point contact devices used in hysteretic synchronization experi-

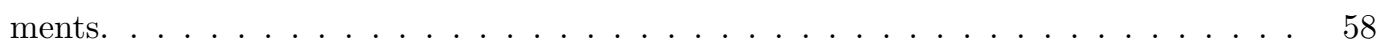

34 Microwave spectra demonstrating hysteretic synchronization. . . . . . . . . . . . 59

35 Dependence of synchronization hysteresis on microwave field and DC current. . . . . 60

36 Dependence of parametric synchronization boundaries on the applied microwave field. 62

37 Oscillation spectra for STNO driven above and below the parametric regime. . . . . 63

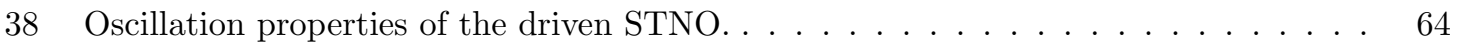

39 Numerical simulations of STNO using the model in the text. . . . . . . . . . . . 66

$40 \quad d V / d I$ for samples $A, B$ under different magnetic fields. . . . . . . . . . . 69

$41 d V / d I$ for samples $A, B$ at the labeled values of $H$, and dependence of dwell times on $I .70$

$42 d V / d I$ for samples $A, B$ at the labeled values of $H$, and dependence of dwell times on $I .70$

43 Distribution of dwell times. . . . . . . . . . . . . . . . . . . . . . . 72

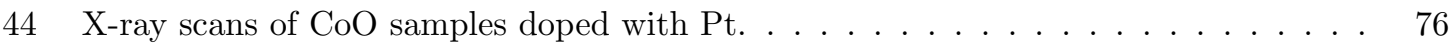

45 In-plane scan for $\mathrm{CoO}$ on $\mathrm{MgO}(100)$ substrate. . . . . . . . . . . . . . . . . . 77 
46 Hysteresis loops for exchange biased samples, after field cooling. . . . . . . . . . 78

47 Temperature dependence of exchange bias and coercivity for polycrystalline and epitaxial samples. . . . . . . . . . . . . . . . . . 80

48 Structural characterization of $\mathrm{Bi}_{2} \mathrm{Se}_{3}$ thin films. . . . . . . . . . . . . . . 84

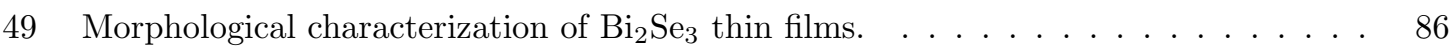

50 XPS spectra of bismuth $4 \mathrm{f}$ doublet peaks and selenium 3d peaks, for a film grown at

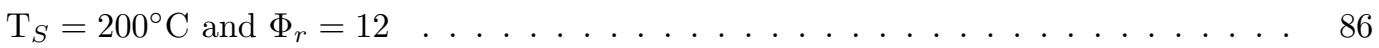

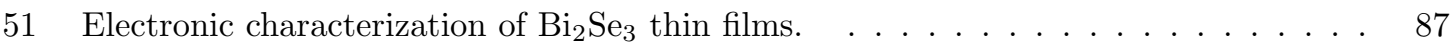

52 XPS spectra of bismuth $4 \mathrm{f}$ doublet peaks for the exfoliated flake. . . . . . . . . . 89

53 XPS spectra of selenium 3d doublet peaks, for the exfoliated flake. . . . . . . . . . 90 


\section{Chapter 1}

\section{Introduction}

\subsection{Motivation}

The invention of silicon electronics has transformed our society, culture, and daily life. For decades transistor density and computing power has doubled every eighteen months. The laws of nature dictate that this pace cannot continue indefinitely, and we are quickly approaching the point of diminishing returns. Therefore, it is imperative to investigate new systems with additional degrees of freedom to continue the cadence of technological growth. In this dissertation, we explore a number of possible avenues to novel information processing and storage technologies.

We begin by detailing our work in the spin torque nano oscillators (STNO). These are some of the smallest known oscillators, and share a number of properties with other oscillating systems. The frequency of oscillation for an STNO is generally $2-10 \mathrm{GHz}$, making them attractive candidates for applications in microwave sensing and emission [1]. Widespread commercial adoption will require optimization of their oscillation characteristics, such as linewidth and power [2]. One avenue of experimental investigation is to mutually synchronize large arrays of oscillators [3]. The first step in this process is the study of synchronization phenomena in a single STNO. We have studied the synchronization characteristics of the STNO to external microwave perturbations, supplied by a microwave antenna. We have performed experiments that demonstrate the oscillation characteristics can be improved by synchronization of an STNO to an external source. 
In addition to nanoscale oscillators, we have also explored the consequences of competing magnetic orders in nanodevices. In particular, interfaces between ferromagnetic and antiferromagnetic materials can result in a phenomenon known as exchange bias [4]. Exchange bias manifests itself as a change in the coercive field of the biased layer, a fact which contributes to its technological utility [5]. While exchange bias may be important in a number of systems, it is not always deisrable. One common problem with nanomagnetic systems is the effect of oxidation of their active layers [6]. Many magnetic materials will exhibit antiferromagnetic ordering following oxidation, possibly resulting in unwanted exchange biasing of the device. We have addressed this issue by the development of a passivation procedure that also serves to prevent shunting of active elements to the device leads. In addition, we have gone beyond merely ameliorating the effects of exchange bias to performing experiments to understand its underlying physical mechanism. By elucidating the underlying physics, it should be possible to optimize device characteristics.

We go beyond the realm of magnetic systems to the growth of thin films of a novel class of materials known as the topological insulators (TI). Recently, TI have emerged as one of the most intensely studied topics in condensed matter physics. These materials possess spin polarized surface states that are robust with respect to environmental and nonmagnetic disorder $[7,8,9]$. Consequently, they are extremely attractive to the spintronics community for possible device applications. The discovery of the topological insulators has raised the possibility of achieving room temperature processing of information by spin alone, in a class of materials that can possibly be incorporated into the current silicon based paradigm [10]. In addition to potential applications, the topological insulators are expected to be a playground for fundamental physics. A number of theoretical works have predicted the existence of previously unobserved quasi-particles. In particular, interfaces of topological insulators with s-wave superconducting materials are expected to host Majorana fermions [11], particles which are their own anti-particle.

We begin our discussion with a treatment of how spin propagates in magnetic systems, followed by a brief introduction to the physics of magnetic ordering in materials. The introduction will conclude with a brief synopsis of the physics of the topological insulators. We will then give a description of the experimental apparatus and techniques used for this work, followed by the original research portion of the text. 

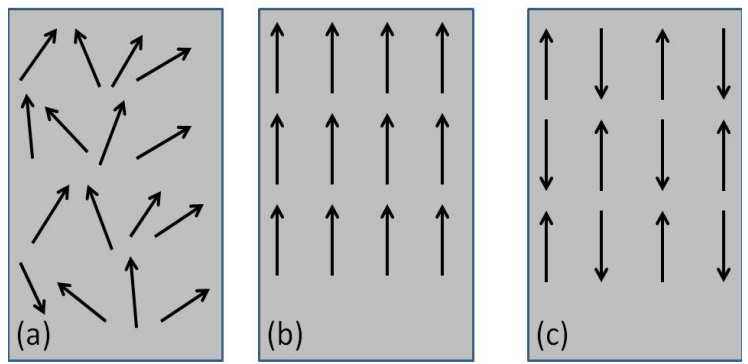

Figure 1: Depiction of magnetic ordering in materials. (a) Highly disordered material (b) Ferromagnetically ordered material, below the transition temperature. (c) Antiferromagnetically ordered material, below the transition temperature.

\subsection{Magnetic Ordering in Materials}

All materials will exhibit a response to a magnetic field, however not all will respond in the same way. Faraday's law implies that as a magnetic field is brought close to a material, there will be an induced current to oppose the change in flux. This response is known as diamagnetism, and is generally a weak response, i.e. it is not the dominant contribution in most systems.

Other materials, palladium for example, will have a large number of itinerant electrons that can align their spins along an externally applied magnetic field. When the field is removed, thermal interactions will randomly orient the spins of the electrons, effectively suppressing the net magnetic moment of the material (Fig. 1 (a)). This phenomenon is known as paramagnetism, and is displayed by a relatively large variety of materials. For other materials, electron-electron Coulomb like interactions and the Pauli exclusion principle will give rise to a spontaneous magnetization in the material that persists even in the absence of an applied field. This phenomenon is known as ferromagnetism, and is shown in Fig. 1 (b).

For other materials, interactions among magnetic moments can favor anti-parallel alignment of adjacent magnetic moments (Fig. 1 (c)). These materials will generally not have a net magnetic moment, but their magnetic structure is far from disordered. This phenomenon is known as antifer- 
romagnetism, and it plays an important role in technological applications.

A portion of our work will be focused on the consequences of interfaces between materials with incompatible magnetic orders. In particular, at the interface between a ferromagnet and an antiferromagnet, new physics can emerge due to the competition between magnetic interactions. In this work, we will be interested in the phenomenon known as exchange bias, as it will serve as a tool to elucidate some of the physics at the interface between incompatible materials.

\subsubsection{Exchange Bias}

Ferromagnetic materials can be characterized by the memory of their magnetic history, a phenomenon known as hysteresis. The magnetization of the sample will be a function not only of the applied field, but the whole history of applied fields. In general, measurements of the magnetization of a ferromagnetic material as a function of applied magnetic field will yield square loops (Fig. 2 (a)). However, in systems that are composed of ferromagnetic and antiferromagnetic layers, this loop can shift along the horizontal axis (Fig. 2 (b)). This effect is a direct consequence of the competing magnetic orders at the interface [4].

The ferromagnetic film will prefer parallel alignment of adjacent magnetic moments, while the antiferromagnetic film will prefer anti-parallel alignments. This results in an interface where the magnetic moments in the ferromagnet are paired against an opposing moment in the antiferromagnetic layer (Fig. $2(\mathrm{c}, \mathrm{d}))$. This coupling can be ferromagnetic or antiferromagnetic in nature; exchange bias results in either case. This coupling creates a local field in the sample that will add or subtract with the applied field to rotate the magnetization of the ferromagnetic layer. This phenomenon can be exploited to pin the magnetic moment of a ferromagnetic layer.

In our work, we explored ways to modify the exchange bias of a well studied system, CoO. In addition, we utilized the exchange bias phenomenon to validate the appropriateness of passivating nanoscale devices with silicon. 

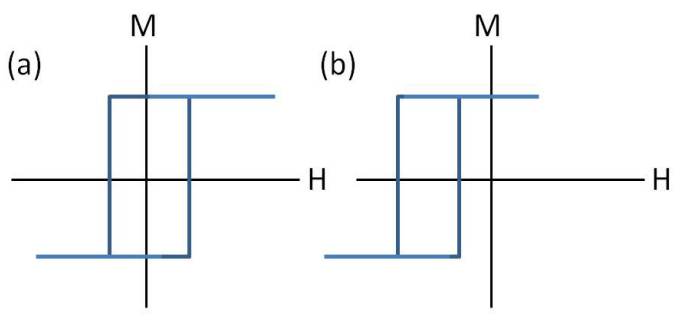

(c)

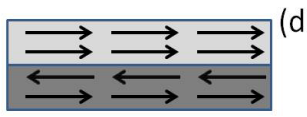

(d)

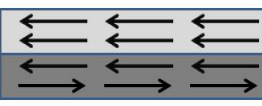

Figure 2: (a) Hysteresis loop for a ferromagnetic material. (b) Hysteresis loop for an exchange biased structure. (c) Idealized depiction of the ferromagnet-antiferromagnet interface, with antiferromagnetic coupling across the interface (d) same as (c) with ferromagnetic coupling across the interface.

\subsection{Spin Dynamics and Transport in Magnetic Systems}

\subsubsection{Magnetization Dynamics and Ferromagnetic Resonance}

Nanoscale magnetic system can exhibit a variety of dynamical behaviors, such as slow / fast reversal and precession of the magnetic moment. Of particular interest for our work here is the phenomenon of precession. To understand this phenomenon, we will begin by considering the case of an electron in a uniform magnetic field executing Larmor precession.

\section{Larmor Precession}

An electron, as a consequence of its intrinsic angular momentum (spin), constitutes a magnetic dipole. The magnetic dipole moment $\boldsymbol{\mu}$, is proportional to its spin angular momentum $\mathbf{S}: \boldsymbol{\mu}=\gamma \mathbf{S}$ where $\gamma$ is the gyromagnetic ratio. When a magnetic dipole is placed in a magnetic field $\mathbf{B}$ it will experience a torque, $-\boldsymbol{\mu} \times \mathbf{B}$ that will attempt to align the magnetic moment parallel to the applied field, similar to the case of a compass needle. The energy of this torque is given by $H=-\boldsymbol{\mu} \cdot \mathbf{B}=-\gamma \mathbf{B} \cdot \mathbf{S}$, which is a minimum when $\mu$ and $\mathbf{B}$ are parallel.

For an electron at rest in a magnetic field oriented along the $z$ direction $\mathbf{B}=B_{0} \hat{z}$. The matrix representation of the Hamiltonian will then be 


$$
\mathbf{H}=-\gamma B_{0} S_{z}=-\frac{\gamma B_{0} \hbar}{2}\left(\begin{array}{cc}
1 & 0 \\
0 & -1
\end{array}\right)
$$

The eigenstates of $\mathbf{H}$ are then:

$$
\chi_{+}=\left(\begin{array}{l}
1 \\
0
\end{array}\right) \quad \text { and } \quad \chi_{-}=\left(\begin{array}{l}
0 \\
1
\end{array}\right)
$$

with eigenvalues $\pm\left(\gamma B_{0} \hbar\right) / 2$, respectively.

There is no time dependence in this problem; Schrödinger's equation reads:

$$
\imath \hbar \frac{\partial \chi}{\partial t}=\mathbf{H} \chi
$$

and the solution can be expressed in terms of the stationary states:

$$
\chi(t)=a \chi_{+} e^{-\imath E_{+} t / \hbar}+b \chi_{-} e^{-\imath E_{-} t / \hbar}=\left(\begin{array}{c}
a e^{\imath \gamma B_{0} t / 2} \\
b e^{-\imath \gamma B_{0} t / 2}
\end{array}\right)
$$

With the constraint that $|a|^{2}+|b|^{2}=1$ the constants a and $\mathrm{b}$ are set through the initial conditions:

$$
\chi(0)=\left(\begin{array}{l}
a \\
b
\end{array}\right)
$$

Without a loss of generality, we write $\mathrm{a}=\cos (\theta / 2)$ and $\mathrm{b}=\sin (\theta / 2)$, yielding:

$$
\chi(t)=\left(\begin{array}{c}
\cos (\theta / 2) e^{\imath \gamma B_{0} t / 2} \\
\sin (\theta / 2) e^{-\imath \gamma B_{0} t / 2}
\end{array}\right)
$$

To understand the evolution of this system, we will calculate the expectation values of the three components of $\mathbf{S}$ : 


$$
\begin{aligned}
\left\langle S_{x}\right\rangle & =\chi(t)^{\dagger} \mathbf{S}_{y} \chi(t)=\left(\begin{array}{ll}
\cos (\theta / 2) e^{\imath \gamma B_{0} t / 2} & \sin (\theta / 2) e^{-\imath \gamma B_{0} t / 2}
\end{array}\right) \times \frac{\hbar}{2}\left(\begin{array}{ll}
0 & 1 \\
1 & 0
\end{array}\right)\left(\begin{array}{c}
\cos (\theta / 2) e^{\imath \gamma B_{0} t / 2} \\
\sin (\theta / 2) e^{-\imath \gamma B_{0} t / 2}
\end{array}\right) \\
& =\frac{\hbar}{2} \sin (\theta) \cos \left(\gamma B_{0} t\right) \\
\left\langle S_{y}\right\rangle & =\chi(t)^{\dagger} \mathbf{S}_{y} \chi(t)=\left(\begin{array}{ll}
\cos (\theta / 2) e^{\imath \gamma B_{0} t / 2} & \left.\sin (\theta / 2) e^{-\imath \gamma B_{0} t / 2}\right) \times \frac{\hbar}{2}\left(\begin{array}{cc}
0 & -\imath \\
\imath & 0
\end{array}\right)\left(\begin{array}{c}
\cos (\theta / 2) e^{\imath \gamma B_{0} t / 2} \\
\sin (\theta / 2) e^{-\imath \gamma B_{0} t / 2}
\end{array}\right) \\
& =-\frac{\hbar}{2} \sin (\theta) \sin \left(\gamma B_{0} t\right)
\end{array}\right. \\
\left\langle S_{z}\right\rangle= & \chi(t)^{\dagger} \mathbf{S}_{y} \chi(t)=\left(\begin{array}{ll}
\cos (\theta / 2) e^{\imath \gamma B_{0} t / 2} & \sin (\theta / 2) e^{-\imath \gamma B_{0} t / 2}
\end{array}\right) \times \frac{\hbar}{2}\left(\begin{array}{cc}
1 & 0 \\
0 & -1
\end{array}\right)\left(\begin{array}{c}
\cos (\theta / 2) e^{\imath \gamma B_{0} t / 2} \\
\sin (\theta / 2) e^{-\imath \gamma B_{0} t / 2}
\end{array}\right) \\
& =\frac{\hbar}{2} \cos (\theta)
\end{aligned}
$$

The physical interpretation of these expectation values is that the electron spin uniformly precesses around the magnetic field, with a frequency $\omega=\gamma B_{0}$. For a magnetic field of about $1 T$, this frequency is of the order 10s of GHz. This phenomenon has broad experimental and technological implications. It serves as the basis for nuclear magnetic resonance as well as electron paramagnetic resonance, both widely used techniques in medicine and fundamental research. The primary difference among these techniques is simply the source of the magnetic moment. For our purposes, we are interested in the behavior of a magnetic film in the presence of an applied field.

\section{Ferromagnetic Resonance}

One special case of Larmor precession is the phenomenon of ferromagnetic resonance (FMR). This technique enables the characterization of relaxation mechanisms in magnetic systems. In a typical FMR experiment, a magnetic film will be subjected to an external microwave field, in addition to a static DC magnetic field. The DC field is swept through a range of values while the microwave 
absorption is measured. Measured spectra will show a peak when the Larmor frequency of the applied field is in resonance with the applied microwaves [12]. These peaks are characterized by their intensity and linewidth, which are indicative of the relaxation mechanisms in the film.

The torque on the magnetic moment is given by: $-\boldsymbol{\mu} \times \mathbf{B}$, which is just the change in the total angular momentum $\mathbf{L}$, thus:

$$
\frac{d \mathbf{L}}{d t}=-\boldsymbol{\mu} \times \mathbf{B}_{i}
$$

where $\mathrm{H}_{i}$ is the internal field. For a ferromagnet with $\mathbf{M}=(N / V)<\mu_{m}>$, we obtain:

$$
\frac{d \mathbf{M}}{d t}=-\gamma \mathbf{M} \times \mathbf{B}_{i}
$$

This expression implies that the magnetization precesses around the field, much like a single electron, and its potential energy with respect to the field does not change. However, what we are lacking is a representation of a relaxation mechanism that will eventually cause the magnetic moment to align along the external DC field; there is no dissipation in the preceeding equation. To account for this, we make the assumption that the rate of relaxation will be proportional to how far out of equilibrium the system is. This will be represented by the difference $M_{S}-M_{z}$, where $M_{S}$ is the saturation magnetization and $M_{z}$ is the z projection of the magnetic moment. We then obtain:

$$
\frac{\partial M_{z}}{\partial t}=-\gamma(\mathbf{M} \times \mathbf{B})_{z}-\frac{M_{S}-M_{z}}{\tau_{1}}
$$

The relaxation time $\tau_{1}$ is the time required for the magnetization to align with the external field. The transverse components of the magnetization will also relax, but will do so at a different rate:

$$
\frac{\partial M_{x, y}}{\partial t}=-\gamma(\mathbf{M} \times \mathbf{B})_{x, y}-\frac{M_{S}-M_{z}}{\tau_{2}}
$$

The uniform precession state can be described as a $k=0$ spin wave superimposed on the $\mathrm{z}$ component of $\mathbf{M}$, due to the fact that the longitudinal components of the magnetization are in phase throughout the precession cycle. A spin wave is a quantized excitation of the spin system with a well defined momentum $|k|=2 \pi / \lambda$ and energy $h \omega=4 \mathrm{JS}(1-\cos \mathrm{ka})$, where $\mathrm{J}$ is the exchange integral (a way 
of accounting for electron-electron interactions), $\mathrm{S}$ is the magnitude of the local spin and a is the lattice constant [14].

The uniform precession state has two mechanisms for relaxation. The first is the decay of the $k=0$ spin wave into a $k \neq 0$ spin wave. This effectively increases the entropy of the system, without decreasing the magnetic potential energy. This process is described by the transverse relaxation time $\tau_{2}$ [14]. The implication for the magnetic moment of the system is that it decays toward the axis; the transverse components vanish.

Another process destroys two $k \neq 0$ spin waves, which are created in the transverse relaxation process, while creating only one new spin wave and a phonon. This process is described by the longitudinal relaxation time $\tau_{1}$, and is a transfer of energy from the spin system to the crystal lattice. This results in a net decrease in magnetic potential energy, due to the alignment of the magnetic moment with the applied field.

If $\tau_{2} \ll \tau_{1}$ then the two processes occur in sequence. If $\tau_{1} \leq \tau_{2}$, the system will relax with constant $|M|$. The time evolution of $|M|$ is described by what is known as the Landau-Liftshitz equation:

$$
\frac{\partial \mathbf{M}}{\partial t}=-\gamma(\mathbf{M} \times \mathbf{B})-\frac{\mathbf{M} \times(\mathbf{M} \times \mathbf{B}}{M^{2} \tau}
$$

However, the preceeding analysis has neglected one quantity that is important in experiments: the sample shape. Including this into the analysis modifies the expression for the magnetic induction field inside the sample: $B_{j}^{i n}=B_{j}^{\text {out }}-N_{j} M_{j}, j=x, y, z$. In the case of a weak microwave field, $M_{z} \approx M_{S}$ and we obtain:

$$
\begin{gathered}
\frac{\partial M_{x}}{\partial t}=-\gamma\left[M_{y}\left(B_{z}-N_{z} M_{z}\right)+M_{z} N_{y} M_{y}\right]=-\gamma M_{y}\left[B+\left(N_{y}-N_{z}\right) M_{S}\right] \\
\frac{\partial M_{y}}{\partial t}=-\gamma M_{x}\left[B+\left(N_{x}-N_{z}\right) M_{S}\right]
\end{gathered}
$$

Please note that the assumption that $M_{z} \approx M_{S}$ precludes the emergence of any nonlinear phenomema. A more detailed treatment would drop this assumption. If we assume a time dependence $e^{i \omega t}$ for the transverse magnetization components, we can solve for the frequency: 


$$
\omega^{2}=\gamma^{2}\left[B+\left(N_{x}-N_{z}\right) M_{S}\right]\left[B+\left(N_{y}-N_{z}\right) M_{S}\right]
$$

Therefore, by knowing the sample shape it is possible to determine a relaxation time from the linewidth $\left((\Delta \omega)_{1 / 2}=1 / \tau_{2}\right)$.

Our experiments with STNO are a variation on this technique. In our experiments, we applied a known static field and then swept the applied microwave frequency, while measuring the microwave emission by the nanoscale magnetic system. The above analysis is simplified and does not account for some of the more subtle properties of these systems. In particular, nonlinear terms which would couple the frequency of oscillation to its amplitude have been neglected. Our spectroscopic measurements enabled us to investigate some of the nonlinear properties of nanoscale magnetic systems, and ascertain how they affect the oscillation properties.

Two additional phenomena will be important for our work in spin torque oscillators. Nanomagnetic devices are comprised of layers of magnetic materials, separated by nonmagnetic conductors. The magnetic orientation of the device layers can be controlled and probed by spin transfer torque, and giant magnetoresistance respectively.

\subsubsection{Giant Magnetoresistance}

\section{Introduction}

Spin dependent transport of electrons in magnetic multilayers will play a central role in our work on microwave dynamics in nanodevices. Perhaps the most widely known consequence of this phenomenon is the giant magnetoresistance effect [13]. The effect manifests itself when passing current through multilayers of magnetic and nonmagnetic materials. It was found that the resistance of the device depended on the relative magnetic orientation of the layers in the sample. In particular, the resistance is found to be maximum when adjacent layers have anti-parallel (antiferromagnetic coupling) orientations and minimum when they are parallel (ferromagnetic coupling).

\section{Toy Model}

To understand the effect we construct a toy model. 

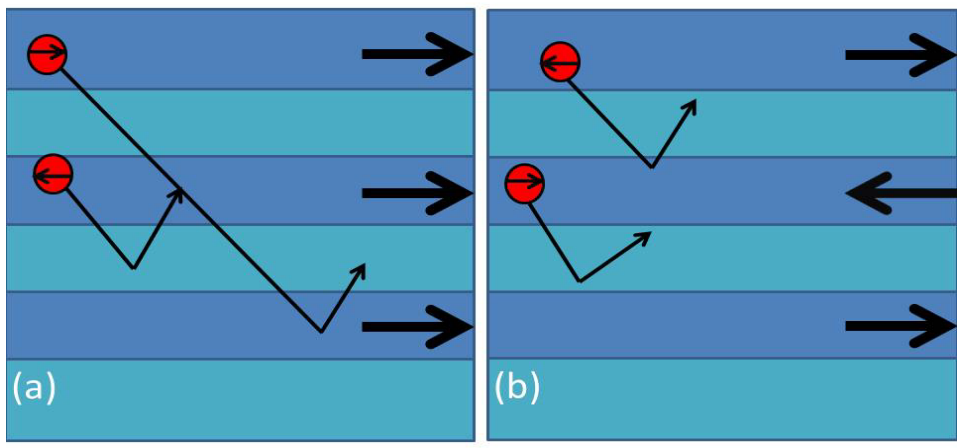

Figure 3: Schematic representation of electron transport in a multilayer structure. Red circle is the electron, the interior arrow indicates its spin orientation. Large arrows show the magnetic moment of the layer. (a) Ferromagnetic coupling (b) Antiferromagnetic coupling.

We start with the assertion that the scattering of an electron incident on a magnetic moment is more likely when its spin is opposite to that of the scattering site. This is a direct consequence of the spin dependent band structure of the ferromagnet. The ferromagnet is described by two different bands, for spin up and spin down electrons. For a ferromagnet with a net magnetic moment, one of these bands will have a larger occupancy (majority band) than the other (minority band). The density of states at the Fermi level is such that the majority band is nearly full, while the minority band has many available states. An incident electron whose spin is aligned anti-parallel to the majority band of the ferromagnet will scatter into the minority band, as it has a large number of available states. For this to happen, the electron must reverse its wave vector; it is effectively reflected at the interface of the ferromagnet.

In general, the scattering site can be magnetic molecules, domain walls or magnetic films. For our purposes, we will consider the case of multilayers of ultra thin magnetic films. For antiferromagnetically coupled multilayers, conduction electrons of both spin orientations with sufficiently long mean free paths will thermally sample a series of weak and strong scattering layers. Spin carriers of each species will thus have comparable mean free paths and resistivities (Fig. 3(b)). For ferromag- 


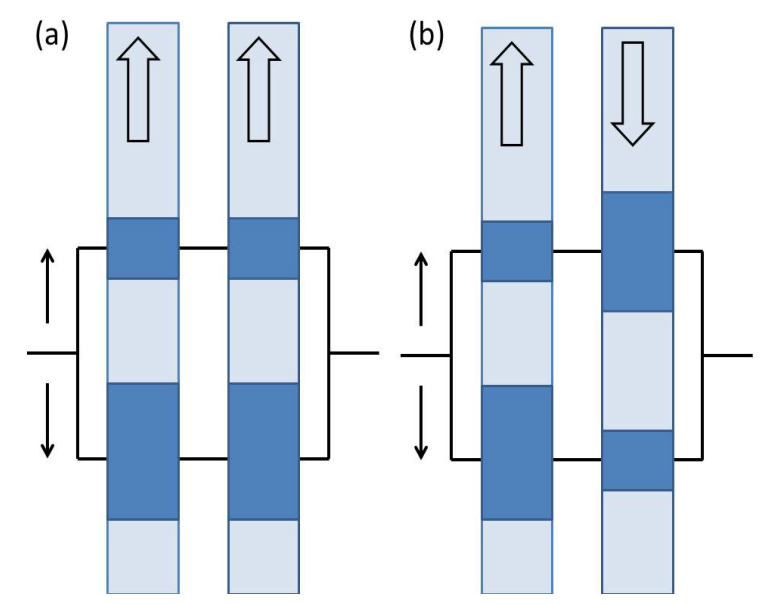

Figure 4: Equivalent circuit for the multilayers in Fig. 3. The upper path is the resistance due to spindependent scattering of spin up electrons, the lower path is for spin down electrons. Shaded regions are the magnetic layers, with the clear arrow indicating the orientation of the magnetic moment. The size of the boxes represent the resistance: the large box mean larger resistance. Spacer layers are omitted for simplicity. (a) Ferromagnetically ordered layers and (b) Anti-ferromagnetically ordered layers.

netically coupled multilayers, conduction electrons with spin orientation parallel to the magnetic moments will experience only weak scattering events. Thus, their mean free path and resistivity is smaller than for those carriers with opposite spin orientation (Fig. 3(a)).

This can also be illustrated by representing the problem as a parallel resistance circuit (Figs. 4(a) and(b)). The blue small (large) shaded boxes represent scattering of carriers of a particular spin in layers with parallel (anti-parallel) magnetic orientation. Here the size of the box represents the magnitude of the resistance:a larger box implies a larger resistance $\mathrm{R}_{L}$ and the smaller boxes have small resistance $\mathrm{R}_{S}$. If we assume that the mean free path of the conduction electrons is longer than the thickness of the spacer layers, then the spin carriers will experience a series of resistivities due to the nonmagnetic $R_{n}=\rho_{n} l_{n} / A_{n}$ and magnetic $R_{m}^{i}=\rho_{m}^{\sigma} l_{m} / A_{m}$ layers as they pass through the multilayer. The index $i=\uparrow$ or $\downarrow$ denotes the spin up or down channel, and $\sigma$ (the open arrows in Figs. 4(b) and 4(a)) denotes the spin in the magnetic layers relative to that of the carriers in the channel [14].

If we compute the total resistance of the equivalent circuits, momentarily neglecting the normal resistivity, we obtain the following equations: 


$$
\begin{gathered}
\frac{1}{R_{A F}}=\frac{1}{R_{L}+R_{S}}+\frac{1}{R_{L}+R_{S}}=\frac{2}{R_{S}+R_{L}} \\
R_{A F}=\frac{R_{S}+R_{L}}{2}
\end{gathered}
$$

Likewise for $\mathrm{R}_{F}$ :

$$
\begin{gathered}
\frac{1}{R_{F}}=\frac{1}{2 R_{S}}+\frac{1}{2 R_{L}} \\
R_{F}=\frac{2 R_{S} R_{L}}{R_{S}+R_{L}}
\end{gathered}
$$

The magnetic contributions are not the whole story, of course. The metallic resistivity can be incorporated in a straight forward way. In the case of ferromagnetic coupling, the resistance in one channel is $R_{i}=2 \rho_{n} l_{n} / A_{n}+2 \rho_{m}^{\sigma} l_{m} / A_{m}$, where $l_{i}$ is the path length in the $n$ or $m$ layer, and $\sigma$ is up for one value of $\mathrm{i}$ and down for the other. Under these conditions, the two parallel spin channels add to give:

$$
R_{F}=\frac{(\alpha+1)(\beta+1)}{\alpha+\beta+2} \frac{\rho_{n} l_{n}}{A_{m}}
$$

where $\alpha=\rho_{m}^{\downarrow} / \rho_{n}$ and $\beta=\rho_{m}^{\uparrow} / \rho_{n}$. In the case of antiferromagnetic coupling, the resistance in each channel is the same, $R_{M}^{\sigma}=2 \rho_{n} l_{n} / A_{n}+\left(\rho_{m}^{\uparrow}+\rho_{m}^{\downarrow}\right) l_{m} / A_{m}$ :

$$
R_{A F}=(\alpha+\beta+2) \frac{\rho_{n} l_{n}}{2} A_{m}
$$

We note that as long as $\rho_{m}^{\uparrow} \neq \rho_{m}^{\downarrow}$ the resistance in the ferromagnetic state will be lower than the antiferromagnetic state, due to shunting of current through the low resistance channel. The GMR ratio is given by:

$$
\frac{\Delta R}{R}=\frac{R_{F}-R_{A F}}{R_{A F}}=-\frac{(\alpha-\beta)^{2}}{\left(\alpha+\beta+2 l_{n} / l_{m}\right)^{2}}
$$

The GMR ratio indicates a decrease in resistance in the ferromagnetic state, limited in magnitude 

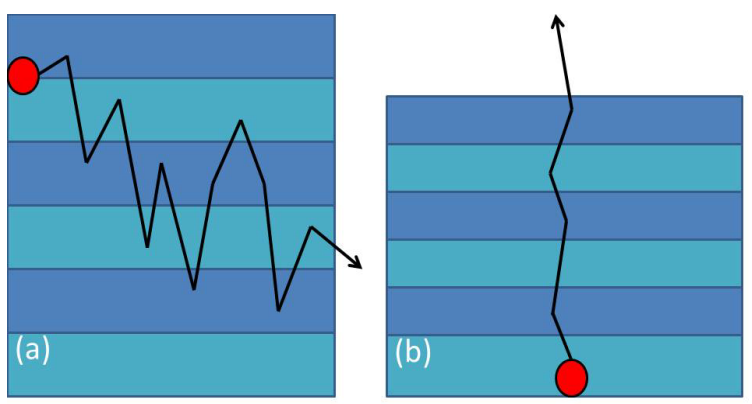

Figure 5: Schematic illustration of current measurement geometries (a) Current in plane and (b) Current perpendicular to plane.

to $100 \%$. The ratio has a quadratic dependence on the difference in scattering from sites of like and unlike spin. Additionally, the GMR effect vanishes in the limit that the nonmagnetic layers are much thicker than the magnetic layers. This is a consequence of collision events in the spacer layer tend to randomize the spin orientation of the current. Once the layer is beyond a certain critical thickness, called the spin diffusion length, the incident spin current will lose its polarization before exiting the conducting layer. All the devices in this work were designed such that the spacer layers are smaller than the spin diffusion length.

\section{Measurement Geometry}

There are two possible ways to pass a current through a multilayer structure. The current can be passed into the plane of the device or film, or it can be injected normal to the surface. These geometries are known as current-in-plane (CIP) and current perpendicular to plane (CPP) respectively. These geometries are shown schematically in Figs. 5(a), and 5(b).

In the CIP geometry, the electron drift direction will be dictated by the in-plane applied electric field. Thermal motion causes the electrons to move transverse to the drift velocity, allowing them to sample the magnetic moment of adjacent layers. The CPP geometry has the advantage that electrons are essentially forced to traverse many interfaces, by the applied electric field. In both geometries the magnetic field is applied in the plane of the sample. 


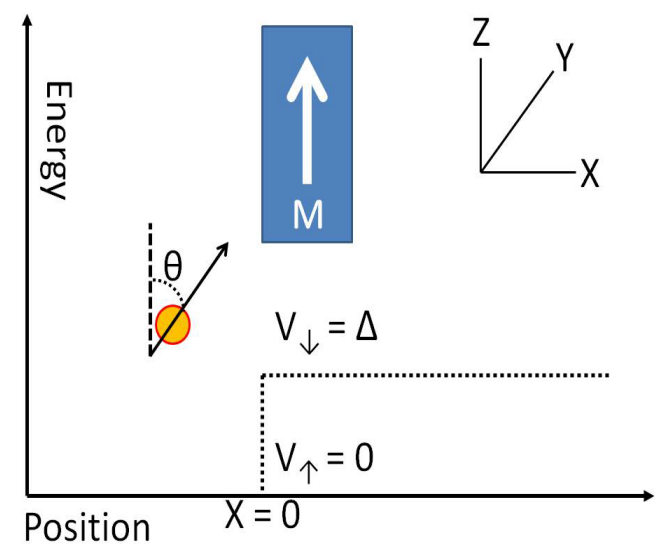

Figure 6: Cartoon depiction of an electron incident on a ferromagnet with moment M. The electron's spin is oriented at angle $\theta$ with respect to the moment of the ferromagnet.

\subsubsection{Spin Transfer Torque}

Thus far we have considered the effect of the magnetic layer on the electronic transport. However, this is only part of the picture. We must develop a description of the consequences of the electron spin on the magnetic layers themselves. As electrons pass through a ferromagnetic material, their spins will tend to align along the local direction of the magnetization. The rotation of the electron spin necessarily means it is losing angular momentum. Since it is a fundamental principle of nature that total angular momentum is conserved, the angular momentum lost by the electron must be transferred elsewhere. As Slonczewski demonstrated [15], this angular momentum is transferred to the magnetic layers in the device. The consequence of this is that the magnetic moment of a ferromagnet can be flipped, or even excited to some dynamical state, by the application of a spin polarized current [1].

We will begin by considering a single electron, moving along the $\hat{x}$ direction, incident on a ferromagnet, with magnetic moment $\mathbf{M}$ aligned along the $\hat{z}$ direction (Fig. 6). We will assume that an electron whose spin is parallel to $\mathbf{M}$ will pass through unaffected, while an electron with antiparallel spin will experience a potential barrier of height $\Delta$. The electron's spin makes an angle $\theta$ with respect to $\mathbf{M}$ and will therefore scatter or transmit with some probability. If the electron is transmitted, it will rotate its spin to be collinear with $\mathbf{M}$. As discussed above, this implies that a 
torque is exerted on the electron and also on the magnetic layer.

Analysis of this problem will require that we define the spin current density, a tensor quantity that defines the flow of the probability density and of the spin angular momentum in real and spin space, respectively.

$$
\mathbf{Q}=\frac{\hbar^{2}}{2 m} \operatorname{Im}\left(\psi^{*} \sigma \times \nabla \psi\right)
$$

where $m$ is the electron mass, $\psi$ is the electron wavefunction and $\sigma$ are the Pauli spin matrices. The electron wavefunction will have the general form:

$$
\psi(x)=\frac{e^{i k x}}{\sqrt{\Omega}}(a|\uparrow>+b| \downarrow>)
$$

where $\Omega$ is the normalization volume. In this problem, there will be two different wave vectors for the different spin orientations. $k_{\uparrow}=\sqrt{2 m E} / \hbar$ and $k_{\downarrow}=\sqrt{2 m(E-\Delta)}$. Since there will be a change in angular momentum, the other quantity of interest is the torque. We know that total angular momentum is conserved, and so any nonequilibrium flux passing through some region of space will necessarily transmit angular momentum. This can be handled in a similar way to Guass' law, by drawing a pillbox around the region of space and calculate the net flux through that region:

$$
\begin{aligned}
N_{S T} & =-\int_{\text {pillbox surface }} d^{2} \mathbf{R} \hat{n} \cdot \mathbf{Q} \\
& =-\int_{\text {pillbox volume }} d^{3} \mathbf{R} \nabla \cdot \mathbf{Q}
\end{aligned}
$$

where $\mathbf{R}$ is the in-plane position, $\hat{n}$ is the interface normal to the pillbox surface. To compute the spin current density, we match the wave functions and derivatives at the interface $(x=0)$. This process will give us the transmitted and reflected portions of the wave function:

$$
\psi_{\text {transmitted }}=\frac{e^{i k_{\uparrow} x}}{\sqrt{\Omega}} \cos (\theta / 2)\left|\uparrow>+\frac{e^{i k_{\downarrow} x}}{\sqrt{\Omega}} \frac{2 k_{\uparrow}}{k_{\uparrow}+k_{\downarrow}} \sin (\theta / 2)\right| \downarrow>
$$




$$
\psi_{\text {reflected }}=\frac{e^{-i k_{\uparrow} x}}{\sqrt{\Omega}} \frac{k_{\uparrow}-k_{\downarrow}}{k_{\uparrow}+k_{\downarrow}} \sin (\theta / 2) \mid \downarrow>
$$

Inserting these definitions into the spin current density yields the following expressions:

$$
\begin{gathered}
\mathbf{Q}_{\text {in }}=\frac{\hbar^{2} k_{\uparrow}}{2 m \Omega}(\sin (\theta) \hat{x}+\cos (\theta) \hat{z}) \\
\mathbf{Q}_{\text {trans }}=\frac{\hbar^{2}}{2 m \Omega} \sin (\theta) \frac{2 k_{\uparrow} k_{\downarrow}}{k_{\uparrow}+k_{\downarrow}}\left\{\cos \left[\left(k_{\uparrow}-k_{\downarrow}\right) x\right] \hat{x}+\cos \left[\left(k_{\uparrow}-k_{\downarrow}\right) x\right] \hat{y}\right\} \\
+\frac{\hbar^{2} k_{\downarrow}}{2 m \Omega}\left\{\cos (\theta / 2)^{2}-\left(\frac{2 k_{\uparrow}}{k_{\uparrow}+k_{\downarrow}}\right)^{2} \sin (\theta / 2)^{2}\right\} \hat{z}
\end{gathered}
$$

These equations imply a number of important facts. First, the transverse (perpendicular to $z$ ) spin component of the reflected spin current density is zero. The total spin current density is continuous at the interface, thus all of the of the transverse component of the incident spin current density is transmitted through the interface. In our analysis, this result follows from our assumption that for spin up electrons do not experience a potential barrier, so their reflection amplitude is zero, and the reflected part of the wavefunction is purely spin down. For models in which both components of spin experience a non-zero potential-energy step, some of the incident $x$ component of the spin current density will be reflected. However, for many of the materials combinations used commonly in metallic GMR devices, such as $\mathrm{Cu} / \mathrm{Co}, \mathrm{Cu} / \mathrm{Ni}$, or $\mathrm{Cr} / \mathrm{Fe}$, one of the spin components actually does have a reflection amplitude close to zero over a large part of the Fermi surface, so it is a reasonable approximation that almost all of the transverse $x$ component of the spin current density will be transmitted into the ferromagnet [1].

Another important consequence of this analysis is the presence of oscillatory terms in the $x$ and $y$ components of the transmitted spin current density. This can be viewed as the precession of the electron spin in the exchange field of the magnet. This feature will be present in any model in which the energy barriers are different for spin up and spin down electrons. In real three dimensional samples this fact is especially important. Electrons will be incident from all parts of the Fermi surface, and will therefore have many different wave vectors. At a given point inside the ferromagnet, 


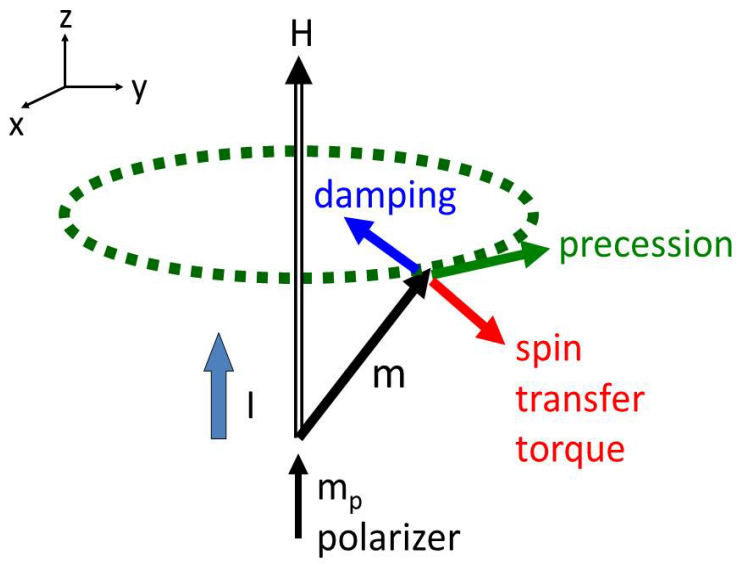

Figure 7: Cartoon depiction of the action of the various torques on the ferromagnet.

the electrons will have traveled a different path length and will accordingly have different phases. The spin part of their wave functions, in general, will add destructively. The net result is that all of the transverse angular momentum is absorbed by the ferromagnet [1].

The consequence of this dephasing is that the total reflected and transmitted spin current densities are approximately collinear with the magnetization of the ferromagnet (along the $z$ direction). No transverse angular momentum flows away from the magnet, so the entire incident transverse angular momentum is absorbed by the magnet, and the spin transfer torque becomes:

$$
N_{S T}=A \hat{x} \cdot\left(Q_{i n}+Q_{\text {refl }}-Q_{\text {trans }}\right) \approx A \hat{x} \cdot Q_{i n}^{\perp}=\frac{A}{\Omega} \frac{\hbar^{2} k_{\uparrow}}{2 m} \sin (\theta) \hat{x}
$$

To incorporate this torque into the equation for the magnetization dynamics, it is typical to add a term $\dot{M} \propto N_{S T}$ to the differential equation for the magnetization dynamics (Eqn. 1.1). In real devices, there will be a polarizing layer that serves to orient the spin direction of the incident electrons, followed by a thin metallic spacer and another ferromagnetic layer whose dynamics we wish to excite.

The differential equation that describes the evolution of the magnetic moment, including the spin torque term, is as follows:

$$
\frac{d \mathbf{M}}{d t}=-\gamma_{0} \mathbf{M} \times \mathbf{H}_{\mathrm{eff}}+\frac{\alpha}{M_{S}} \mathbf{M} \times \dot{\mathbf{M}}-\Gamma I \hat{\mathbf{M}} \times\left(\hat{\mathbf{M}} \times \mathbf{M}_{\text {fixed }}\right)
$$




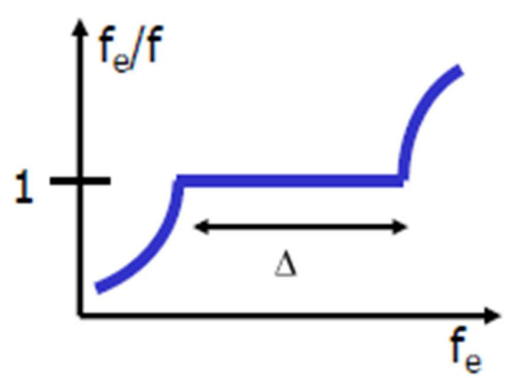

Figure 8: Depiction of synchronization. Horizontal axis is the perturbing frequency. Vertical axis is the ratio of the perturbing to the oscillator frequency.

The first term represents precession about the effective field, which includes the applied field as well as crystalline and shape anisotropy fields. The second term is the damping term, similar to what was discussed in the section on magnetization dynamics. The final term is the action of the spin transfer torque. The action of all these forces on the moment of the magnet is represented schematically in Fig. 7. In principle, the above equation can be solved for the state of the ferromagnet at any time. This equation serves as the basis for all theoretical investigations into spin transfer driven phenomenon.

\subsection{Synchronization}

As we have seen, nanoscale magnetic systems can execute uniform precesion, a type of oscillatory motion. Like many oscillating systems, they can be driven by periodic external perturbations. Under certain conditions, these perturbations can result in the synchronization (or entrainment) of the spin torque oscillator to the external driving force. Synchronization is easiest to observe when plotting the ratio of the driving frequency to the oscillation frequency of the entrained system (Fig. 8). When the driving frequency $\omega_{e}$ is close to the instantaneous oscillator frequency $\omega$, the two will become exactly equal over some range of perturbing frequencies. The coupling strength between the two systems must be relatively weak, otherwise they could be considered as a single system.

Additionally, they must both be autonomous systems that can oscillate independently. It is this qualification that distinguishes synchronization from resonance. The more familiar phenomenon of 
resonance also occurs when a periodic system is driven by an oscillating perturbation. For example, consider the case of a pendulum with a magnet at its end. If we push the pendulum, it will begin to oscillate. This system, due to frictive forces, will eventually come to rest, i.e. it is not an autonomous system. If we operate an $\mathrm{AC}$ electromagnet near the pendulum, and time it so that it repels the pendulum at the halfpoint in its cycle, we will be able to maintain the oscillation of the pendulum. By varying the frequency of the current through the magnet, we can also change the frequency of the oscillating pendulum. This would appear to be synchronization, however it is not. If we decouple the systems, for instance by placing mu metal between them, the pendulum will come to a rest. It is therefore not an autonomous system and cannot be entrained [56].

\subsection{Non-trivial Spin Textures: Topological Insulators}

\subsubsection{Introduction}

Topological insulators are a new quantum state of matter that is characterized by a bulk bandgap that is traversed by spin polarized surface states. The band structure of these materials is similar to graphene, posessing an odd number of Dirac cones in the Brillouin zone [7]. This new state is realized in certain materials due to the presence of large spin orbit coupling and time reversal symmetry. To date, combined theoretical and experimental efforts have uncovered the existence of two classes of TI: the 2D and 3D topological insulators. The 2D topological insulator is synonymous with the quantum spin Hall insulator, which is related to the quantum Hall state of matter. The hallmark of this phase is spin polarized currents along the sample edges. In contrast, the $3 \mathrm{D}$ topological insulator supports 2D spin polarized Dirac fermions on its surface, and is an entirely new state of matter. In this work, we will focus on the 3D topological insulators.

We begin our discussion with a fundamentally related phenomenon, the quantum Hall effect. In the ordinary Hall effect, a magnetic field is applied perpendicular to a film while passing a current through the material. Measurements of the resistivity and transverse voltage will yield information about the carrier types and their concentration. In particularly pure semiconductors, at large magnetic fields and low temperatures, quantum mechanical effects can become important [16]. The quantum Hall effect manifests itself as a quantization of the Hall resistivity and concurrent 

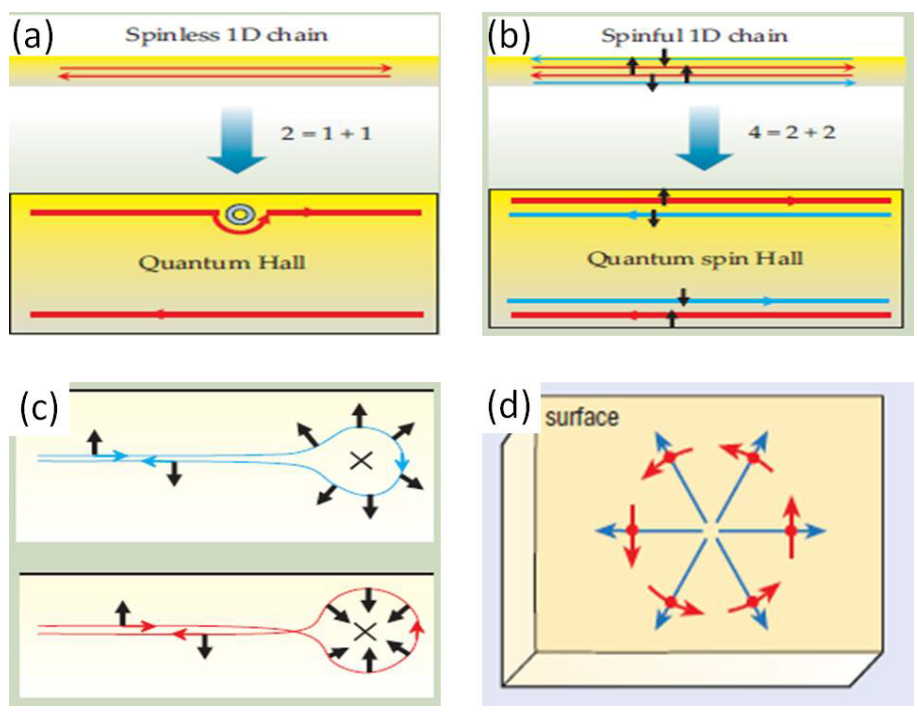

Figure 9: (a) Cartoon representation of the quantum Hall effect. The equation $2=1+1$ symbolizes the two channels, one forward and one backward moving. (b) The quantum spin Hall effect. The equation $4=2+2$ symbolizes the four channels, one forward spin up, one forward spin down and likewise for the backward channels. (c) Cartoon representation of scattering in the quantum spin Hall phase. (d) Cartoon representation of the surface of a topological insulator. From reference [16].

spikes in the resistivity. This is a consequence of the fact that the density of states in the bulk of the material goes to zero, causing the charge carriers to conduct along the sample edges. These edge carriers have no available channels for scattering, and hence flow without dissipation. This yields the minima in the resistivity of the material. This situation is depicted in Fig. 9 (a). This would be useful for devices, were it not for the stringent requirements on sample temperature, purity, and magnetic field.

It is therefore reasonable to ask: Is it possible to relax these requirements and still obtain dissipationless transport? The answer to this question lays in what is known as the quantum spin Hall effect. In certain materials, large spin-orbit coupling can play the role of an applied magnetic field. In the quantum spin Hall effect, there are four channels for charge carriers. Two forward moving, one with spin up and one with spin down, and likewise for two backward moving channels (Fig. 9 (b)). This results in a net propagation of spin along each edge. In this system, transport is robust (dissipationless) as long as there are no magnetic impurities present.

When a charge carrier encounters a non-magnetic impurity, it will simultaneously traverse both 
possible paths around the impurity (both clockwise and anti-clockwise), and these paths will interfere. Going clockwise around the impurity, the spin rotates by $\pi$, going anti-clockwise results in a spin rotation of $-\pi$ (Fig. 9 (c)). The phase difference between these two paths is $2 \pi$. Recall that when the spin part of a fermion wave function is $\propto e^{i \hbar \psi / 2}$, therefore a rotation by $2 \pi$ acquires a phase of -1 . The two paths therefore interfere destructively and effectively suppress backward scattering. This argument will not hold in the presence of magnetic impurities, as they break the time reversal symmetry of the system.

While we have eased the restriction on the external magnetic field, the quantum spin Hall state still requires low temperatures that are accessible only by exotic cooling methods, such as dilution refrigeration. The realization of dissipationless spin polarized currents at room temperature had to wait until the discovery of the topological insulators.

In the topological insulators, the surface charge carriers are spin-momentum locked (Fig. 9 (d)), meaning that motion in real space determines the orientation in spin space. This unique property persists even at room temperature. This makes topological insulators compelling candidates for room temperature spintronic applications.

\subsubsection{Experimental Realization}

The above description may give the impression that transport measurements are a natural technique to verify the existence of the topologically protected surface states. However, it was soon realized that the bulk conductance of these materials effectively overwhelms any contribution from the surface states. It was therefore apparent a new technique would be required to demonstrate this new phase of matter.

Angular resolved photoemission spectroscopy (ARPES) measurements are particularly well suited for measurements of the dispersion of the surface states. This technique is similar to XPS, the primary difference being the ability to perform angular resolved measurements. A typical ARPES setup will have the ability to resolve the momentum vector of the ejected electrons, and will thus be able to distinguish surface state electrons from bulk electrons. This is a consequence of the fact that the surface electrons will not have a momentum component perpendicular to the surface, while bulk electrons will. By measuring the energies and momenta of the ejected electrons, it is possible 


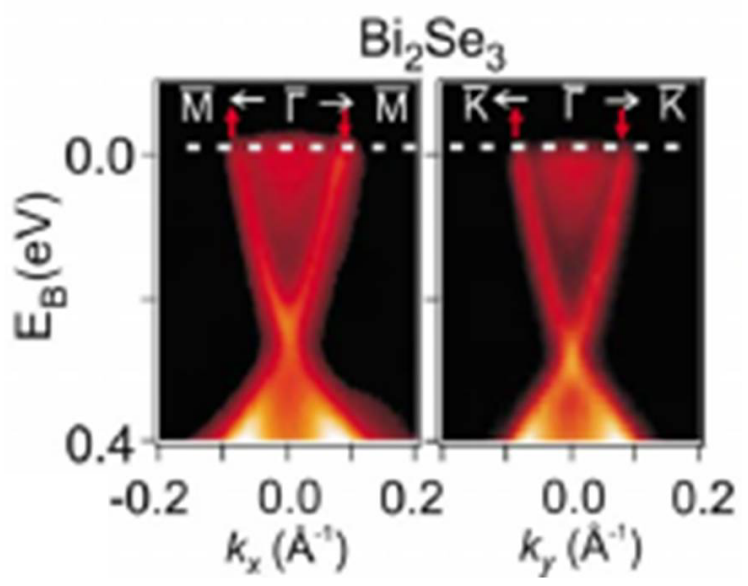

Figure 10: ARPES spectrum of a cleaved bulk crystal of $\mathrm{Bi}_{2} \mathrm{Se}_{3}$. The spectra reveals the existence of a single Dirac cone. From reference [7].

to reconstruct the band structure of the material.

Following the theoretical prediction of their existence [17], the 3D topological insulating phase was first discovered in bulk crystals of $\mathrm{Bi}_{1-x} \mathrm{Sb}_{x}$ [18]. ARPES measurements revealed the presence of a single Dirac cone in the first Brillouin zone, as well as demonstrating their highly spin polarized texture [19]. This effectively validated the theoretical predictions, and ignited a firestorm of inquiry in the condensed matter community.

\section{Second-Generation Materials}

$\mathrm{Bi}_{1-x} \mathrm{Sb}_{x}$ was the first discovered 3D topological insulator, however it is not the most ideal. The bulk bandgap is only of order $50 \mathrm{meV}$ [18], and hence bulk conduction will still suppress contributions from the surface states. The community sought new materials with the requisite band structure and larger bulk band gaps. Theoretical work suggested that other bismuth based materials, such as the thermoelectric $\mathrm{Bi}_{2} \mathrm{Se}_{3}$ should display topological insulating behavior and have a significantly larger band gap [8].

ARPES measurements on $\mathrm{Bi}_{2} \mathrm{Se}_{3}$ revealed the existence of a nearly idealized Dirac cone (Fig. 10), with a band gap of approximately $300 \mathrm{meV}$ [20]. Room temperature measurements also show a single Dirac cone, that is stable with respect to environmental doping with $N O$ [21]. These factors, combined with the fact that $\mathrm{Bi}_{2} \mathrm{Se}_{3}$ is a stoichiometric compound instead of an ordered allow, has 
propelled $\mathrm{Bi}_{2} \mathrm{Se}_{3}$ to the forefront of research in topological insulators.

One problem remains, however. $\mathrm{Bi}_{2} \mathrm{Se}_{3}$ is still a narrow band-gap semiconductor with a nearly metallic bulk. Selenium vacancies that cause deviations from nominal stoichiometry donate electrons, rendering the as-grown materials n-type [22]. Typical carrier concentrations range from $10^{17}$ $\mathrm{cm}^{-3}$ to $10^{20} \mathrm{~cm}^{-3}$, with the lowest concentrations achieved by doping with light elements, such as calcium [22]. However, this results in agglomerations of the dopant materials, making interpretation of the transport data ambiguous.

An additional route to observing unambiguous signals from surface transport involves growth of reduced dimensional structures and films. By physically reducing the bulk of the material, it should be possible to observe the surface states electronically. A number of groups have begun growing these materials by molecular beam epitaxy $[10,23,24,25,26]$, as it allows direct control over the growth parameters. It is therefore conceivable that growth by MBE could enable the reduction of defects and open up new classes of experiments that directly exploit the surface states.

This possibility served as the motivation of our work to grow $\mathrm{Bi}_{2} \mathrm{Se}_{3}$ by molecular beam epitaxy. Previous growth studies of $\mathrm{Bi}_{2} \mathrm{Se}_{3}$ had utilized silicon substrates. However, thermally activated transport of intrinsic carriers in silicon complicates the interpretation of transport measurements. Therefore, we elected to utilize a substrate that would not interfere with transport measurements. The growth of this material on insulating $\mathrm{Al}_{2} \mathrm{O}_{3}$ will be discussed in detail in chapter 5 . 


\section{Chapter 2}

\section{Experimental Techniques}

\subsection{Thin Film Growth and Nanofabrication}

\subsubsection{Molecular Beam Epitaxy}

Molecular beam epitaxy (MBE), shown schematically in Fig. 11, is a technique utilized to grow high quality thin films. This technique relies on the impingement of molecular beams of elemental or compound materials on the substrate surface, where the constituents can arrange themselves according to the underlying substrate. Direct control of the growth conditions, such as incident flux and substrate temperature, enable the growth of epitaxial crystals with atomically sharp interfaces. In addition, a typical MBE setup will incorporate the use of various in - situ characterization techniques, such as reflectivity high energy electron diffraction (RHEED) [27] and Auger electron spectroscopy (AES) [28]. These techniques enable characterization during and after deposition, to provide real time feedback on growth conditions.

Typical MBE vacuum systems will have background pressures in the $10^{-10}$ torr range, enabling the growth of high quality semiconductors and other materials in a contaminant free environment. This level of purity is generally not available with other growth methods, such as vapor phase epitaxy or sputtering, and this makes MBE a natural choice for research environments. The mean free path 


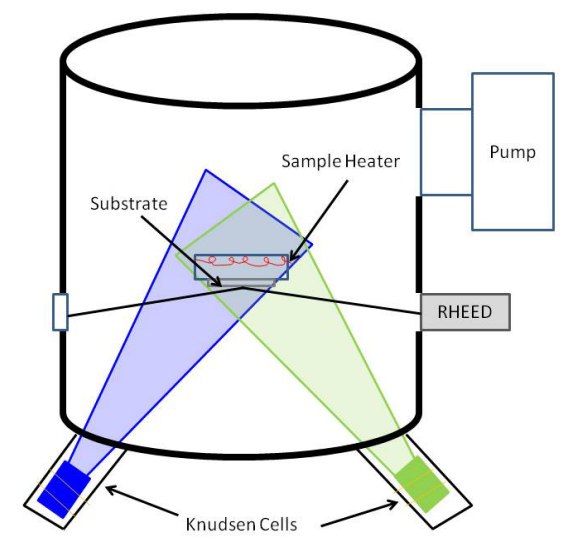

Figure 11: Representation of a typical molecular beam epitaxy system.

in a vacuum system is given by [29]:

$$
L_{B}^{-1}=\sqrt{2} \pi n_{b} d_{b}^{2}+\pi n_{g} d_{b g}^{2} \sqrt{1+\left(v_{g} / v_{b}\right)^{2}}
$$

where $n_{b}, d_{b}$, and $v_{b}$ are the average concentration, diameter and average velocity of the source atoms, respectively. The terms with the subscript $g$ refer to the respective terms for the residual gasses in the chamber (i.e. hydrogen, water, etc.), and $d_{b g}=\left(d_{b}+d_{g}\right) / 2$. For systems with pressure in the $10^{-10}$ range, the mean free path is of order $10^{3}$ meters, meaning that constituent atoms travel ballistically to the substrate without colliding with one another.

Constituent materials and compounds are typically effused from Knudsen cells (k-cells), which operate by purely thermal means. Source materials are placed in an non-reactive crucible, and radiatively heated by one or a series of resistive filaments. Thermocouples are utilized to provide temperature feedback, enabling the use of PID (proportional, integral, and derivative) control to achieve temperature stability of $\pm 0.1^{\circ} \mathrm{C}$. Deposition rates are typically one monolayer per second, which is slow enough to accomodate surface migration of the constituent atoms.

There are at least two possible growth modes for MBE. When growing $I I I-V$ semiconductor compounds, it is typical to deposit one material at a time, creating layers of materials by sequentially shuttering the sources for a period of time. The amount of time the sources are shuttered will depend 
on the growth rate of the materials. This technique has enabled the growth of high quality InGaN quantum well films [30]. Alternatively, both sources can be opened simultaneously. This variation relies on the surface kinetics of the source materials to form an ordered crystal. We employed the latter technique for the growth of high quality $\mathrm{Bi}_{2} \mathrm{Se}_{3}$ films [31].

\subsubsection{Thermal Evaporation}

When precise control over sample stoichiometry and a contaminant free environment are not required, a simpler technique can be employed. Thermal evaporation is another example of physical vapor deposition, similar to MBE. The primary differences are the background pressure during deposition and the ability to monitor the deposition rate during film growth. Typical thermal evaporator systems achieve background pressures in the range $10^{-6}$ to $10^{-7}$ torr, where the mean free path is of the order meters instead of kilometers. Moreover, evaporators do not come with separate load locks, hence the system must be opened for each use and is generally not baked out following opening. Without baking, the adsorbed layer of water will have to desorb from the system over time, resulting in higher background pressures than in an MBE system.

Another difference is the way in which the source material is evaporated. Unlike MBE, where k-cells are utilized, thermal evaporators generally use metallic boats to evaporate source materials. For the fabrication of nanodevices, we utilized aluminum boats to evaporate elemental aluminum, to act as a mask for the patterning of nanopillars. Boats are typically discarded after each use, while MBE crucibles are more robust and are only changed following a catastrophic failure or when changing source material.

Finally, the deposition rates for thermal evaporation are much higher. As surface roughness and epitaxy are not paramount concerns, high throughput is enabled by increasing the deposition rate of the source materials. Typically, several Angstroms per second are deposited with a thermal evaporator, as opposed to sub-Angstrom per second deposition rates with MBE. This makes thermal evaporation an ideal technique for mask patterning in nanoscale devices, when the rate of fabrication is important. 


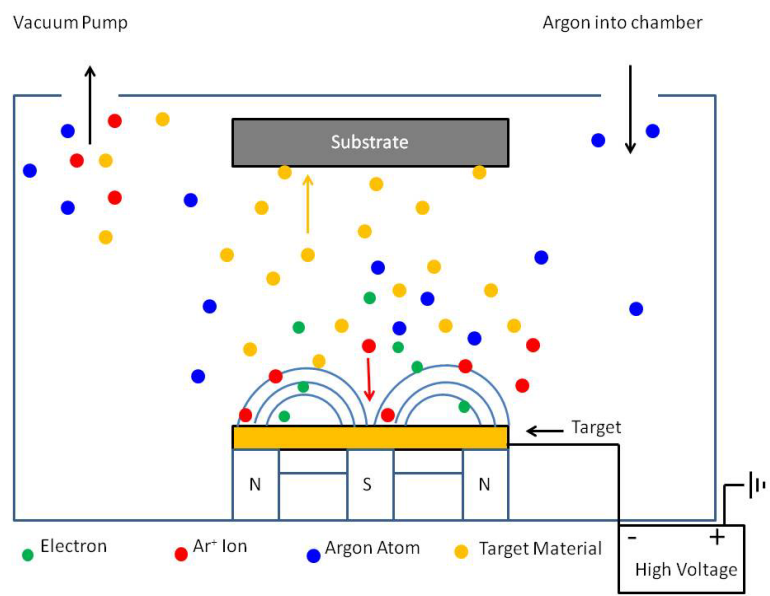

Figure 12: Cartoon depicting the DC magnetron sputtering process.

\subsubsection{Magnetron Sputtering}

Perhaps the most prevalent form of thin film deposition is sputtering(represented schematically in Fig. 12). In contrast to the previous techniques, where heat is utilized to eject source material, in sputtering a plasma is used to ablate source material from a target. The target is held at a negative voltage, typically hundreds of volts, while an inert gas is introduced into the vacuum system. Electrons are stripped from the mediating gas, producing a plasma of positive gas ions and negative electrons. The ions are then accelerated toward the surface of the target, and once they collide they eject the target material as well as causing the emission of secondary electrons. The secondary electrons are accelerated away from the cathode (target) and execute cylclotron orbits around the magnetic field, supplied by an array of permanent magnets. The free electrons strip bound electrons from the mediating gas, effectively feeding the reaction.

The ejected target atoms travel diffusively throughout the vacuum system, and a portion of them will collide with the substrate surface, depositing a film. In contrast to molecular beam epitaxy, the deposition is not directional, meaning that source atoms will be incident from all directions. This generally results in smoother surfaces, but makes controlling the surface kinetics more difficult. Consequently, sputtering is an effective method for quickly depositing smooth films, although they will generally lack registry with the substrate material. 
DC magnetron sputtering is a useful technique for deposition of metals, which would otherwise be difficult to evaporate. However, for insulating materials the technique encounters difficulties. Since an insulating target will effectively shield the applied voltage, enormous DC voltages would have to be supplied. To counter this, application of a radio frequency voltage will prevent charge buildup on the insulating substrate, facillitating plasma ignition.

For our nanodevices, DC sputtering was utilized to deposit all the conducting and magnetic elements. Silicon and silicon oxide were sputtered using an RF source at $50 \mathrm{~W}$ of incident power. These techniques were used in conjunction with thermal evaporation to fabricate nanoscale magnetic devices.

\subsubsection{Electron-beam Lithography}

Lithography refers to the general process of writing patterns in a polymer film. In electron-beam lithography, a collimated beam of electrons is used to perform the writing, as opposed to ultraviolet light. A typical e-beam writing system will be very similar to a scanning electron microscope, with the addition of a way to blank the beam to prevent unwanted exposure (Fig. 13).

One critical consideration in e-beam lithography is the choice of polymer. The first parameter to consider is the tone of the resist. Tone comes in two varieties: positive and negative. When positive resist is exposed to an electron beam, the polymer chains dissociate and can thus be easily removed by a developing solution. This gives a "hole" in the regions that are exposed to the beam. Exposure of negative resist to an electron beam causes the polymer chains to strengthen, making them insoluble in the developing solution. This results in a "bump" in the exposed areas. For all of our lithography, we used positive resist polymers.

In our nanofabrication procedure, we employed the use of a bilayer resist, with each polymer in the bilayer having a different sensitivy to the electron beam. The underlayer is comprised of methyl-methacrylate (MMA), which has a high sensitivity to the beam current (typical dose of $125 u \mathrm{C} \mathrm{cm}^{-2}$ at $30 \mathrm{kV}$ ). The overlayer is made of poly methyl-methacrylate (PMMA), with a typical dose of $200 u C \mathrm{~cm}^{-2}$ at $30 \mathrm{kV}$. The purpose of using a resist with a higher sensitivity for the underlayer is to exploit the proximity effect. Lateral broadening of the pattern will result in undercut, meaning that the pattern will be broader at the base than the top. This allows better 


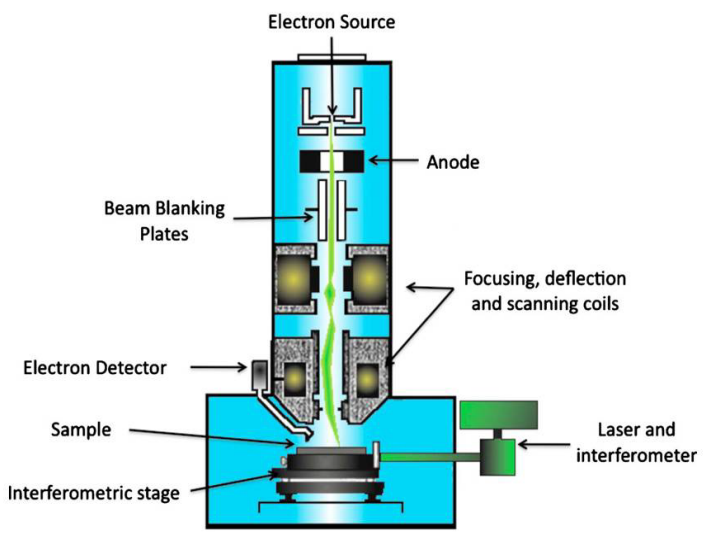

Figure 13: Schematic of a typical electron-beam lithography system. Shown are the electron gun, and all the optics for focusing, scanning, and blanking the beam. From reference [32].

adhesion of films to the surface, and prevents unwanted loss of deposited materials during liftoff. The polymers are applied with a dropper, spun at $\approx 5000 \mathrm{rpm}$ and then baked for several minutes at $170^{\circ} \mathrm{C}$ to outgas the solvent that keeps the polymers in liquid state.

Following exposure, the samples are removed from the SEM and placed in a solution of MIBK:IPA that etches the polymer in the exposed regions. The sample is then inspected under an optical microscope, to ensure that the pattern has been adequately developed. Once the samples have been further processed, either by thermal evaporation or magnetron sputtering, the excess materials and polymer are removed with acetone, leaving the deposited materials only in the areas exposed to the electron beam.

\subsubsection{Argon Ion Milling}

Following lithography and deposition of the requisite materials, at certain points it becomes necessary to etch away deposited materials to create a pattern. In the case of nanopillar fabrication, it is necessary to etch away the magnetic and conducting materials to reveal the nanopillar pattern. There are two primary methods for etching materials: chemical and dry etching. In chemical etching, acids or bases are used to chemically remove layers of materials. One disadvantage of this approach is that different materials will be etched at different rates, or not at all, with the same 
etchant. Moreover, it is unavoidable that the etchant fills the entire available space, and this can cause undesired etching of exposed areas. In general, this is difficult to control and can lead to a significant degree of variation between samples.

Alternatively, a beam of atoms can be used to ablate the magnetic and conducting layers. This is accomplished by ionizing a heavy inert gas, such as Argon, and then using one or a series of grids, held at a potential, to accelerate and collimate the beam of Argon ions. Once beyond the grid, a neutralizer filament can be used to neutralize the Argon beam, to reduce charging effects. The beam then impinges on the sample surface, removing atoms in the process. This effectively etches exposed areas, while the beam directionality insures that there is no unintended etching of unwanted areas [33]. This technique enables repeatable fabrication, and can be finely tuned by the use of a quartz crystal monitor to determine etching rates for individual materials in a multilayer stack.

In our fabrication procedure, Argon ion milling served a two fold purpose. First, it was utilized to mechanically clean the sample surface by removing unwanted adsorbates that may interfere with subsequent sputter deposition. Second, it was used to pattern the nanopillar shape, by simultaneously etching through the aluminum mask and underlying magnetic / conducting multilayer stack. We were able to determine precise milling rates by sputter coating the quartz crystal monitor with the same materials present in the multilayer, and then ion milling the crystal monitor to determine the precise etching rate. Using this method, we were able to successfully mill down to any desired interface, thus enabling the patterning of both nanopillars as well as point contact devices.

\subsection{Sample Characterization and Measurements}

\subsubsection{Atomic Force Microscopy}

Atomic force microscopy (AFM) is a powerful technique that enables characterization of surface topography. In a typical AFM setup, a cantilever, approximately $150 \mu m$ in length, holds a sharp nanoscale tip that is kept some distance above the surface of interest. Either the tip mounting or the sample mounting are attached to a piezoelectric material, for which an applied voltage yields a known vertical displacement. Additional piezos are used to raster the sample stage in the $\mathrm{x}-\mathrm{y}$

direction. An infrared laser is reflected off the back surface of the cantilever, and the reflection 
generates a voltage across a diode array. There are presently two mechanisms for AFM feedback, known as contact and tapping mode. Schematics of typical AFM setups are shown in Figs. 14 (a),14 (b)

In contact mode, the tip is held at a constant vertical displacement from the surface. Short range interactions between the tip and the sample surface will cause the tip to deflect, and the piezo is adjusted to bring the tip to the desired sample-tip distance. These adjustments can be used to create a topographic map of the sample surface. This was the original feedback mechanism for the AFM, however it has a number of drawbacks. For samples that are scanned in air, there will be a layer of adsorbed water on the surface; when the tip comes into contact with this layer, surface tension can cause the tip to be drawn to the surface, creating aberrant data. An additional complication arises when scanning soft samples: the tip can easily damage the surface of the sample.

In non-contact, or tapping mode, the cantilever is oscillated at its natural frequency, typically on the order of $50-100 \mathrm{kHz}$. As the tip oscillates, it will intermittently make contact with the sample surface. As it does, it will lose energy due to attractive/repulsive surface-tip interactions, and will thus change its oscillation amplitude. This change in amplitude is registered by the diode array, and can be utilized to create a topographic map of the surface of interest. Reduced interactions between the tip and surface result in longer tip lifetimes as well as reduced damage to the sample surface.

\subsubsection{X-ray Diffraction}

Among the most widely used techniques for materials characterization is x-ray diffraction. Discovered in the early $20^{t h}$ century by Bragg, this effect was quickly exploited and utilized in nearly every area of material science. The utility of the technique is a consequence of the wavelength of the light: $\mathrm{x}$-rays are in the angstrom regime, which is comparable to the lattice spacing of most materials. This makes x-ray diffraction ideally suited for crystallographic characterization of materials.

Crystalline materials are composed of stacked planes of regular arrays of atoms; this fact enables both reflection of the x-rays as well as interference from scattering off different atomic planes. These two conditions are essentially all that is required to observe x-ray diffraction [35]. Reflections of a monochromatic beam from adjacent planes will have a path difference of $2 d \sin (\theta)$, and in order for them to constructively interfere, this path difference must be an integral number of wavelengths of 

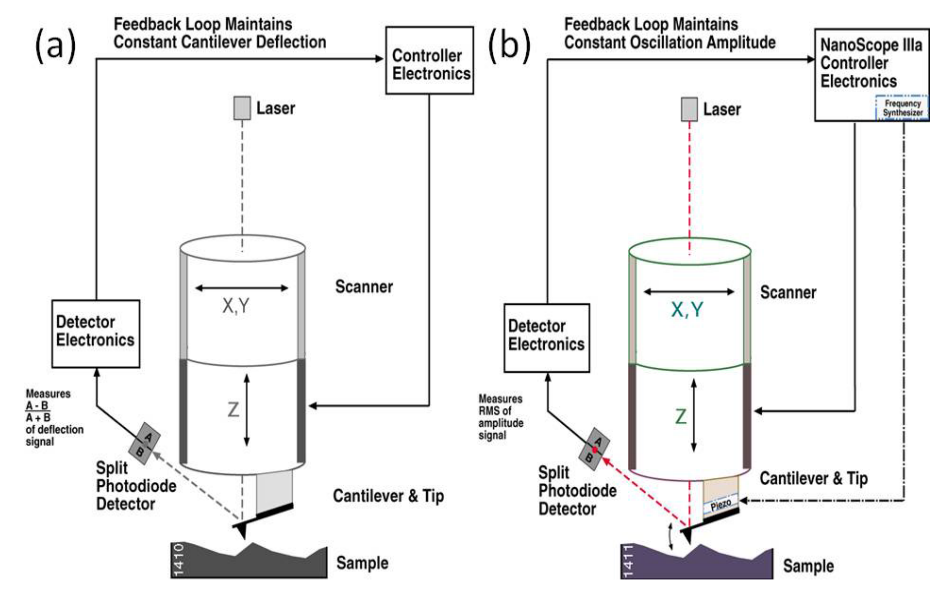

Figure 14: (a) Schematic representation of a typical contact mode atomic force microscope setup. (b) Schematic representation of a typical non-contact, or tapping mode, atomic force microscope setup. From reference [34].

the incident light. This is known as Bragg's law [36]:

$$
n \lambda=2 d_{h k l} \sin (\theta)
$$

Where the $d_{h k l}$ is the spacing between adjacent lattice planes $(h k l) . \lambda$ is the wavelength of the incident radiation, and $\theta$ is the angle of the reflection with respect to the surface normal and $\mathrm{n}$ is the order number. A schematic representation of this process is shown in Fig. 15.

There are a variety of scans that enable one to acquire information about the crystal quality. A representation of a typical four circle x-ray diffractometer, similar to the one used for all the diffraction scans in this work, is shown in Fig. 16. All scans require that the sample be located at the center of rotation of the diffractometer. For out-of-plane measurements, the center of rotation involves having the rotation angle $\chi=90^{\circ}$, where the zero of $\chi$ is defined as having the normal to the sample surface oriented vertically. In-plane measurements require adjustments away from $\chi=90^{\circ}$ or by adjusting $\omega$ and $2 \theta$ such that the desired reflection can be measured [38].

Determination of crystal phases and lattice spacings can be made by performing $\omega-2 \theta$ scans. This 


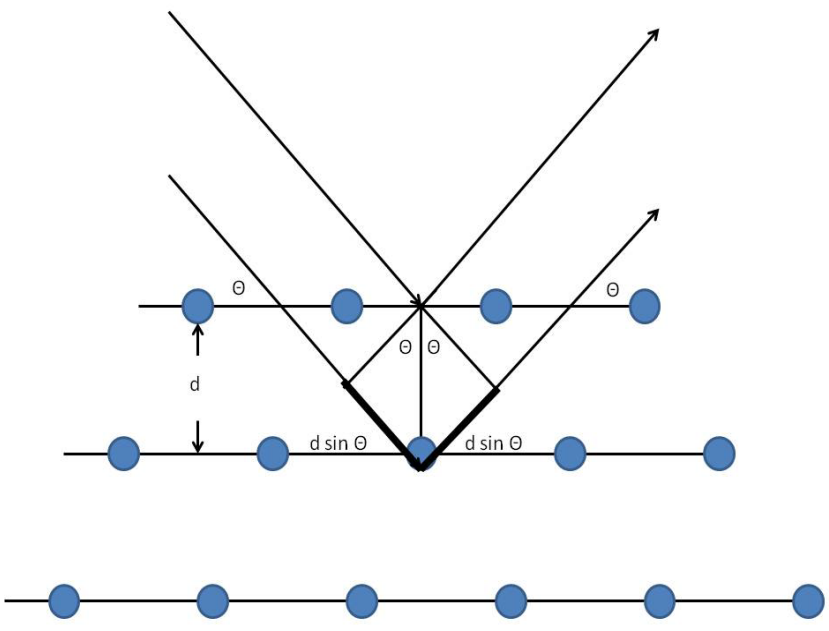

Figure 15: Representation of Bragg's law.

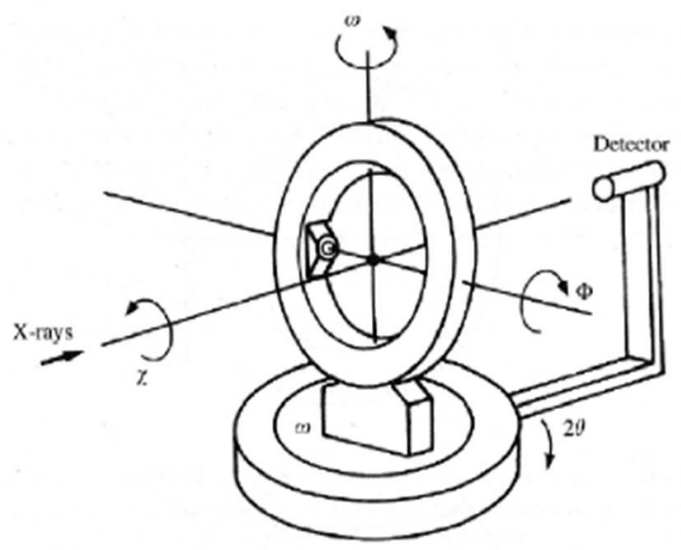

Figure 16: Illustration of a typical four circle diffractometer. From reference [37]. 
is done by recording the diffracted intensity as the incident beam is scanned through a wide range of angles, keeping $\omega$ fixed at one half the value of $2 \theta$. Estimation of crystal quality can be done by performing $\omega$ scans, also known as rocking curves. This involves fixing $2 \theta$ while $\omega$ is scanned about the ideal value of $\frac{1}{2} 2 \theta$. Keeping the intensity of the instrument constant enables meaningful comparison of the full width at half maximum of the scans, for different samples, to make qualitative statements of the impact of growth conditions on crystal quality [38].

\subsubsection{X-ray Reflectivity}

When the angle of incident $\mathrm{x}$-rays are small $\left(\lesssim 10^{\circ}\right)$ the $\mathrm{x}$-ray reflections become sensitive to a number of factors, such as the interfacial roughness and the film thickness [39].

Intensity oscillations in the $\omega-2 \theta$ are commonly referred to as Kiessig fringes, which is a consequence of interference between reflections from different interfaces [40]. Parratt developed a recursive algorithm in which the electric field in each layer of a sample is modeled [41]. To account for the interfacial roughness, Névot and Croce introduced an exponential decay factor, proportional to the square of the vertical roughness, at each interface [42]. Parrat's algorithm was used to determine the roughness and thickness for all the films in the $\mathrm{Bi}_{2} \mathrm{Se}_{3}$ portion of this work.

\subsubsection{Reflectivity High Energy Electron Diffraction}

Reflectivity high energy electron diffraction has emerged as a powerful technique for in-situ characterization of thin films, providing real time feedback on crystal growth. A typical RHEED system can be seen in Fig. 17. RHEED exploits the small wavelength of relativistic electrons to obtain diffraction patterns of films during deposition. Typical energies of incident electrons are 5 - $40 \mathrm{keV}$, and their wavelength is approximated by:

$$
\lambda \approx \frac{12.247}{\sqrt{V\left(1+10^{-6}\right)}}(\AA)
$$

Where $\mathrm{V}$ is the accelerating voltage in Volts. The accelerated electrons therefore have a wavelength of $0.2-0.06 \AA$. The small incident angle guarantees that there is a very small component of the wavevector perpendicular to the sample surface. This reduces the contribution from planes parallel to the sample surface. The penetration depth of the electrons, for these energies and angle of 


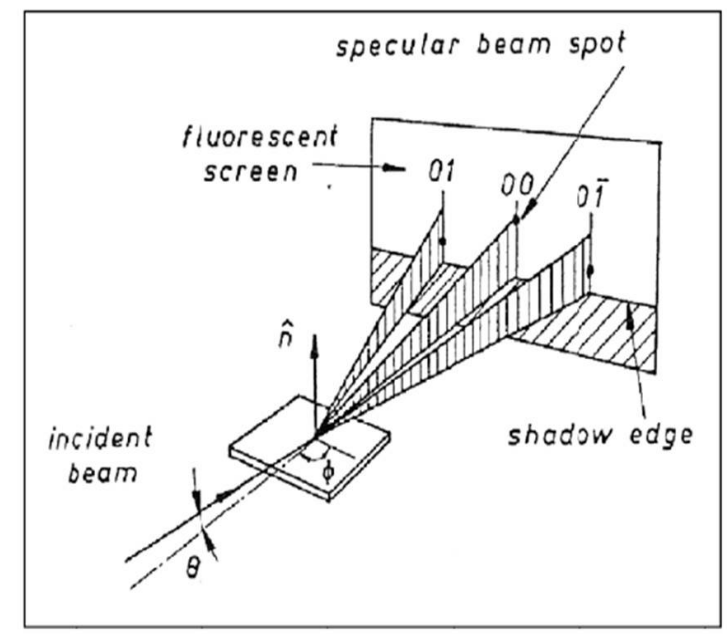

Figure 17: Schematic of a typical RHEED setup. From reference [29].

incidence, is only a few atomic layers [43]. This makes RHEED a highly surface sensitive technique. If the sample is crystalline, then diffraction patterns can be observed that obey Bragg's law (see the section on x-ray diffraction).

RHEED was used in our characterization of ultra thin $\mathrm{Bi}_{2} \mathrm{Se}_{3}$ films. Data was recorded during deposition, generating plots of intensity as a function of time. We observed a phenomenon known as RHEED oscillations, that explicitly demonstrates the layer by layer growth of the thin films. The period of the oscillations will correspond to the deposition time for one layer in the film.

\subsubsection{X-ray Photoelectron Spectroscopy}

X-ray photoelectron spectroscopy (XPS) is a basic materials characterization technique that enables the experimenter to determine the elemental composition of a material. Data is obtained by irradiating the sample with a beam of x-rays and measuring the energy and intensity of the emitted electrons. This technique is highly surface sensitive, with the penetration depth being of the order $10 \mathrm{~nm}$ at most. Measurements are typically performed in an ultra high vacuum environment.

The technique cleverly exploits the photoelectric effect. The energy of the incident x-rays is known, while the energy of the emitted electrons is measured. This enables the determination of the binding energy of the electrons through the following relation: 


$$
E_{\text {binding }}=E_{x-\text { ray }}-E_{\text {electron }}-\phi
$$

Where $\phi$ is the work function of the spectrometer (a known quantity). The acquired spectra consists of the intensity (or number) of electrons hitting the detector as a function of their measured energy. Since the energy of the atomic levels is known, this enables the determination of the electronic states of the emitted electrons, i.e. $1 \mathrm{~s}, 2 \mathrm{~s}, 2 \mathrm{p}$, etc. To determine relative atomic composition of a material, the intensity of the spectra is integrated and then multiplied by the ratio of the appropriate relative sensitivity factors [44].

All the films in the $\mathrm{Bi}_{2} \mathrm{Se}_{3}$ series were analyzed using XPS. We acquired spectra of the $\mathrm{Bi} 4 f_{7 / 2}$, $\operatorname{Bi} 4 f_{5 / 2}$, Se $3 d_{5 / 2}$, and Se $3 d_{3 / 2}$ states. The relative atomic concentration was calculated using the following equation:

$$
\rho=\frac{I_{S e}}{I_{B i}} \frac{S_{B i}}{S_{S e}}
$$

Where $\mathrm{I}_{x}$ is the integrated intensity of both doublet peaks, for element $\mathrm{x}$, and $\mathrm{S}_{S e}=0.44$ and $\mathrm{S}_{B i}=9.848$. These surface sensitivity factors were acquired from the commercially available XPS software analysis package.

\subsubsection{The Hall Effect}

A common technique for determining the carrier densities in metals and semiconductors exploits what is called the Hall effect. Named for its discoverer, E.H. Hall observed that when a current is passed through a metal in a uniform magnetic field, charge accumulates on the sides of the sample, generating a voltage. Measurements of this voltage enable characterization of the electronic properties of the material under study.

The experiment is depicted in Fig. 18. An electric field $\mathrm{E}_{y}$ is applied along the sample, causing a current density $\mathrm{j}_{y}$. In addition, a magnetic field $\mathbf{H}$ points in the positive $z$ direction. Consequently, the Lorentz force [36]

$$
-\frac{e}{c} \mathbf{v} \times \mathbf{H}
$$




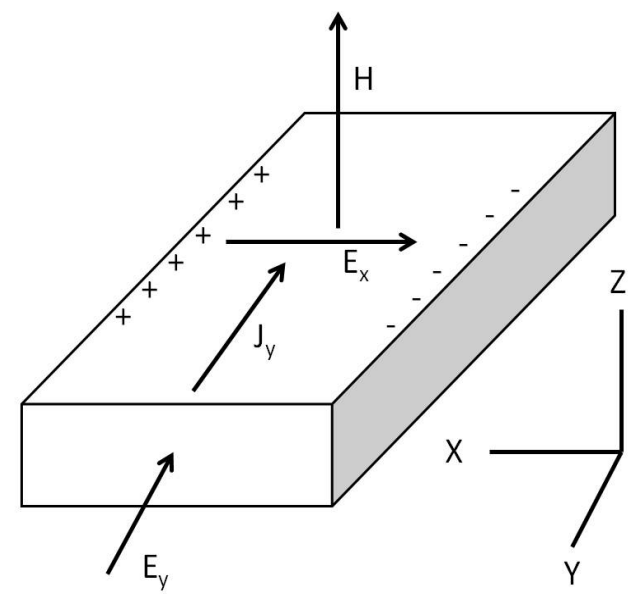

Figure 18: Schematic view of a Hall experiment.

will deflect electrons in the negative $x$ direction. The electrons are constrained by the sides of the sample, where they father. As they accumulate, an electric field is generated that opposes their further movement. Therefore, in equilibrium, the only net current is in the $y$ direction. There are two quantities of interest in this problem, the magnetoresistance and the Hall coefficient.

The magnetoresistance is simply the ratio of the field along the wire to the current density:

$$
\rho=\frac{E_{y}}{j_{y}}
$$

And the Hall coefficient relates the transverse field to the current and magnetic field:

$$
R_{H}=\frac{E_{x}}{j_{y} H}
$$

The sign of the Hall coefficient thus gives the sign of the carriers. This simple analysis, however, is not sufficient to determine the magnitude of the charge density. To cast the Hall coefficient in a more useful form, one must analyze the components of the Lorentz forces acting on the charge carriers and apply the steady state conditions. After some manipulation, one arrives at the result:

$$
R_{H}=\frac{1}{n e c}
$$


In practice, this means that one applies a known voltage to the length of the sample and measures the resulting transverse voltage and logitudinal current, for a given magnetic field. These quantities are sufficient to calculate the Hall coefficient, and thus determine the sign and magnitude of the charge carriers. Hall measurements were performed on all the $\mathrm{Bi}_{2} \mathrm{Se}_{3}$ films in this study.

\subsubsection{Electronic Measurements}

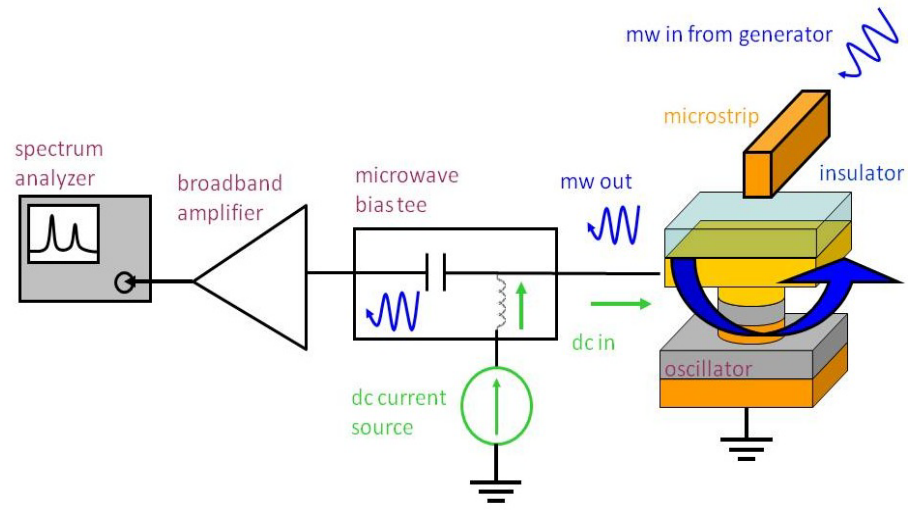

Figure 19: Schematic view of the microwave spectroscopy measurement.

A number of electronic and microwave measurements were performed in the work on the spin torque nano-oscillators. An overview of the microwave experimental setup is shown in Fig. 19. AC and DC current were supplied from a commercially available Keithley current source and passed through a microwave bias tee. Microwave signals were supplied by a commercial Anritsu signal generator, with a $2 \mathrm{GHz}$ to $20 \mathrm{GHz}$ output signal range. Microwave emission was measured by an Anritsu signal generator, typically with bandpass of $5 \mathrm{MHz}$ for measurements of unsynchronized oscillation, and bandpass of $300 \mathrm{kHz}$ for synchronized measurements. AC voltage (differential resistance) measurements were performed by a Signal Recovery lock-in amplifier.

Horizontal magnetic field was supplied from a commercially available GMW magnet that is secured to a rotatable platform, enabling rotation about the vertical axis. The horizontal magnet 

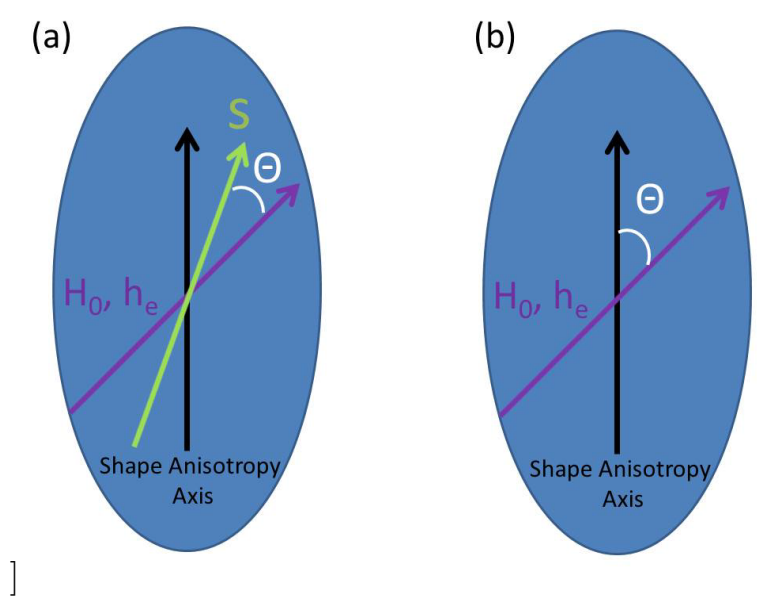

Figure 20: Depiction of the orientation of applied microwave and static fields. Black arrow indicates patterned layer's easy axis. Microwave magnetic field $h_{e}$ is oriented at $45^{\circ}$ with respect to the easy axis. (a) Angle $\Theta$ is between static DC field and symmetry axis s of the oscillation trajectory. (b) Angle $\Theta$ is between the patterned layer's easy axis and the static DC field.

was capable of supplying fields up to $1 \mathrm{~T}$. Vertical magnetic field was supplied through a homemade electro-magnet capable of producing fields up to $\approx 1200$ Oe. The combintation of the two magnets enabled three dimensional field rotation. Samples were mounted in a commercially supplied Advanced Research Systems cryostat, capable of reaching temperatures as low as 5K.

\section{van der Pauw Technique}

To determine both the sample resistivity and carrier concentration, measurements in the van der Pauw geometry were performed. As originally devised by van der Pauw [46], one uses an arbitrarily shaped sample containing four very small contacts placed on the periphery (preferably in the corners) of the sample. A schematic of a rectangular van der Pauw configuration is shown in Fig. 21. Panels (a) and (b) demonstrate the geometry for determining the resistivity and/or magneto-resistance (depending on the absence or presence of magnetic field), while panels (c) and (d) show the geometry for determining the carrier concentration in the Hall measurements.

The sheet resistance can be determined through the following relation:

$$
e^{-\pi R_{A} / R_{S}}+e^{\pi R_{B} / R_{S}}=1
$$

where $\mathrm{R}_{A}=\mathrm{V}_{24} / \mathrm{I}_{13}$ and $\mathrm{R}_{B}=\mathrm{V}_{12} / \mathrm{I}_{34}$. 


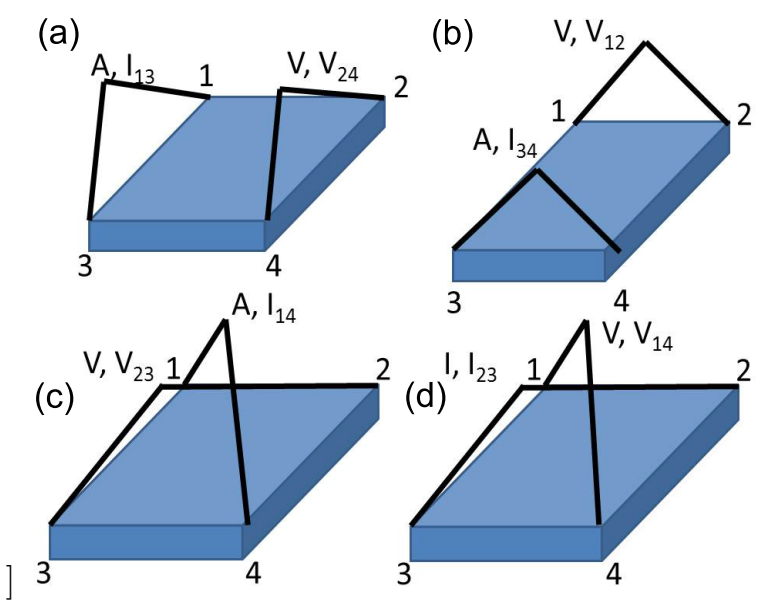

Figure 21: Depiction of the van der Pauw technique. $\mathrm{I}_{x x}$ and $\mathrm{V}_{x x}$ denote current and voltage measurements between terminals xx. (a) (b) are the geometries for resistance / magnetoresistance measurements. (c) (d) are the geometries for Hall measurements.

Whereas the carrier concentration can be obtained through the following relation:

$$
n_{s}=\frac{I B}{V_{H}}
$$

where $\mathrm{V}_{H}=\mathrm{V}_{23}$ or $\mathrm{V}_{34}$ in Fig. 21 (c) and (d), respectively.

In practice, the sample is wired on all four corners and all sixteen permutations are measured. Averaging the measured voltages enables the elimination of any measurement errors that result as a consequence of contact misalignment. Measuring all sixteen permutations enabled a determination of resistivity as well as carrier concentration in the $\mathrm{Bi}_{2} \mathrm{Se}_{3}$ ultra thin films. In the CoOPt study, only the measurements corresponding to panels (a) and (b) in Fig. 21 were performed, as Hall data was not needed. 


\section{Chapter 3}

\section{Synchronization of Spin Torque Nano-Oscillators}

\subsection{Device Fabrication}

\subsubsection{Nanopillars for Current Induced Switching}

Multilayers $\mathrm{Py}(1) \mathrm{Cu}(50) \mathrm{Py}(20) \mathrm{Cu}(8) \mathrm{Py}(5) \mathrm{Au}(25)$, from bottom to top with thicknesses in nm, were deposited on oxidized silicon at RT by sputtering at base pressure of $5 \times 10^{-9}$ Torr, in 5 mTorr of purified Ar. Lithographically patterned $100 \mathrm{~nm} \times 50 \mathrm{~nm}$ Al nanopillar (Fig. 23) served as a mask for the subsequent $\mathrm{Ar}$ ion milling, which removed the multilayer down to the middle of the $\mathrm{Cu}(8)$ spacer, leaving the $\mathrm{Py}(20)$ polarizer unpatterned with dimensions of several micrometers. For sample type $A$, a $30 \mathrm{~nm}$-thick undoped Si layer was then sputtered without breaking vacuum. For sample $B$, $30 \mathrm{~nm}$ of $\mathrm{SiO}_{2}$ was deposited by reactive Si sputtering in 5 mTorr of $\mathrm{Ar}_{2} \mathrm{O}_{2}$ 80:20 mixture. We will show below that this procedure had an oxidizing effect similar to exposure to air, while avoiding surface contamination and variations of atmospheric conditions.

Subsequent ion milling at $3^{\circ}$ with respect to the surface removed the insulating cap from the nanopillar. Finally, a $120 \mathrm{~nm}$ thick top $\mathrm{Cu}$ lead was deposited after etching in 1:10:1000 solution of $\mathrm{HNO}_{3}: \mathrm{HF}: \mathrm{H}_{2} \mathrm{O}$ to clean the nanopillar surface. This cleaning step was more efficient for samples $B$, resulting in somewhat lower resistance. However, the magnetoresistances (MR) of the two sample 
types were similar. Test samples with a Si spacer but no pillar yielded resistances exceeding $2 \mathrm{k} \Omega$, suggesting that $30 \mathrm{~nm}$ thick $\mathrm{Si}$ is sufficiently insulating not only for metallic nanopillars, but also for tunnel junctions. Lock-in measurements of differential resistance $d V / d I$ were performed in a pseudo four-probe geometry, by superimposing a $20 \mu \mathrm{A}$ rms ac current on the dc current $I$. Magnetic field $H$ was applied along the nanopillar easy axis. Two samples of each type were tested with similar results.

\subsubsection{Nanopillars for Fractional Synchronization}

Devices with bottom-to-top structure $\mathrm{Cu}(40) \mathrm{Py}(15) \mathrm{Cu}(8) \mathrm{Py}(3.5) \mathrm{Cu}(60)$, where thicknesses are in $\mathrm{nm}$ and $\mathrm{Py}=\mathrm{Ni}_{80} \mathrm{Fe}_{20}$, were fabricated by e-beam lithography. The $\mathrm{Py}(3.5)$ layer was patterned into a $100 \mathrm{~nm} \times 50 \mathrm{~nm}$ nanopillar (Fig. 23), while the $\mathrm{Py}(15)$ layer was left extended with dimensions of several micrometers. The extended Py layer serves the role of the polarizer as it has a much larger total magnetic moment and is not easily flipped, for the ranges of fields used in this study. The $\mathrm{Py}(3.5)$ layer serves as the free layer, as its relatively small volume generates a small magnetic moment. Magnetic oscillations of the nanopillar were induced by a dc bias current $I_{0}>0$ flowing from the polarizer to the free layer. The microwave driving field $h_{e}$ was generated by a $300 \mathrm{~nm} \times 250 \mathrm{~nm}$ $\mathrm{Cu}$ microstrip antenna fabricated on top of the nanopillar. It was oriented at $45^{\circ}$ with respect to its easy axis, and electrically isolated from $\mathrm{STNO}$ by a $\mathrm{SiO}_{2}(50)$ layer.

\subsubsection{Point Contacts for Hysteresis}

Devices with bottom-to-top structure $\mathrm{Cu}(40) \mathrm{Py}(3.5) \mathrm{Cu}(8) \mathrm{Co}_{50} \mathrm{Fe}_{50}(10) \mathrm{Cu}(60)$ were fabricated on sapphire substrates patterned into coplanar striplines, by the procedure for sample B described in the section on nanopillars for current induced switching. All thicknesses are in nanometers, $\mathrm{Py}=\mathrm{Ni}_{80} \mathrm{Fe}_{20}$. The polarizing CoFe layer was patterned into a $100 \mathrm{~nm} \times 50 \mathrm{~nm}$ nanopillar (Fig. 23), while the free $\operatorname{Py}(3.5)$ layer was left extended with dimensions of several micrometers (Fig. 33 (a)), thus forming a device geometry similar to the magnetic point contact studied in Ref. [47]. Magnetic auto-oscillations of the extended Py layer in the point contact area were induced by a dc bias current $I_{0}>0$ flowing from CoFe. We performed measurements in a more common device geometry with a nanopatterned free layer, but have not observed hysteresis in these devices. The absence of hysteresis 


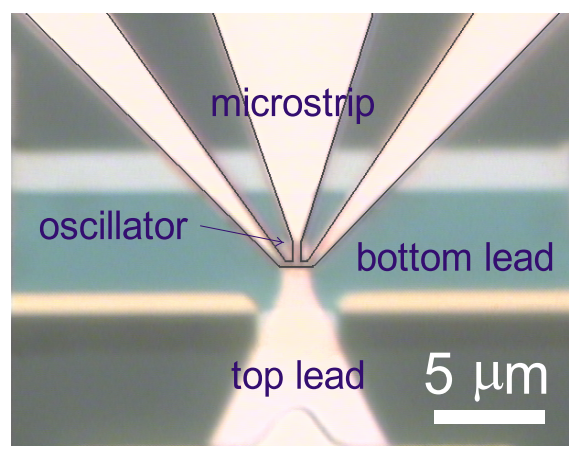

Figure 22: Optical image of completed device.

in the nanopatterned free layers is likely due to smaller nonlinearity and a stronger contribution from fluctuations that generally suppress hysteretic phenomena.

\subsection{Microwave Stripline Calibration}

To enable the application of a large driving signal, we employed the use of a microwave magnetic field. The microwave field was generated by a $300 \mathrm{~nm}$ wide and $250 \mathrm{~nm}$ thick $\mathrm{Cu}$ microstrip fabricated on top of the spin torque oscillator, and electrically isolated from it by a $\mathrm{SiO}_{2}(50)$ layer (Fig. 33 (a)). From Ampere's law, the magnetic field produced by a current carrying strip is proportional to $\frac{1}{w i d t h}$. Therefore, the width of the microstrip was chosen to maximize the magnetic field produced, while simultaneously enabling sufficient heat dissipation to prevent burning of the device. The microstrip was oriented at $45^{\circ}$ with respect to the nanopillar easy axis.

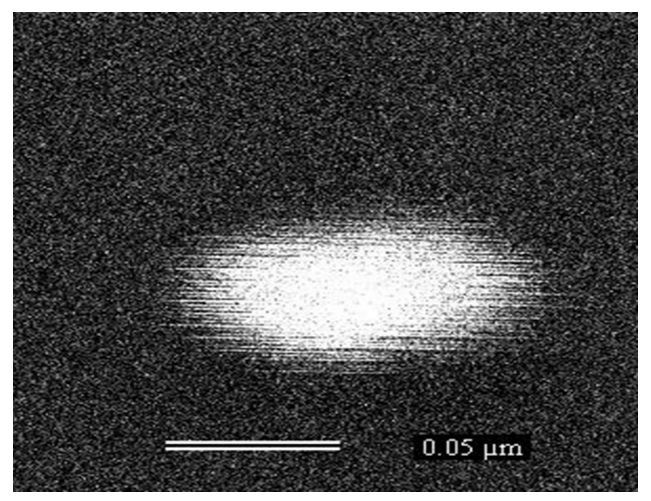

Figure 23: Scanning electron micrograph image of a 100x50 nm pillar. 

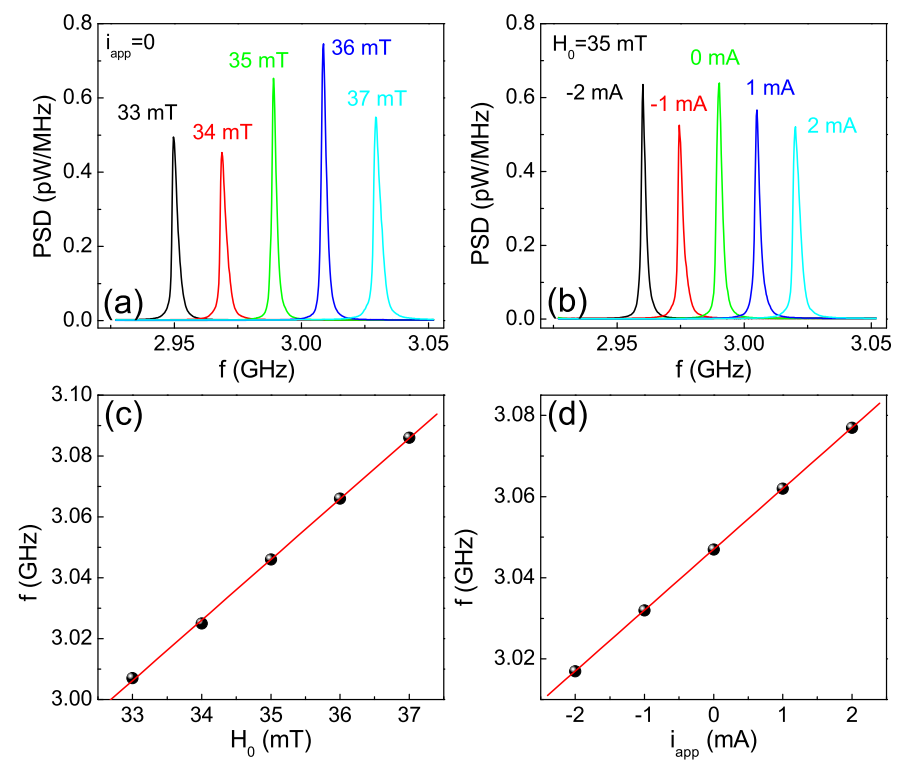

Figure 24: Calibration of microwave field produced by microwave stripline. (a) Dependence of autooscillation frequency on applied DC magnetic field, with no current applied through the microstrip. (b) Dependence of auto-oscillation frequency on applied current through the microstrip, with fixed DC magnetic field. (c) Auto-oscillation frequency (Black symbols) vs. DC magnetic field. (d) Auto-oscillation frequency (Black symbols) vs. applied DC current. Red line is linear fit.

\subsubsection{Point Contacts}

Calibration of the magnetic field produced by the microstrip proved to be a non-trivial problem: we were unable to utilize a separate sensor to determine the field. However, in the small field regime the oscillator frequency has a linear dependence on applied DC field. We were therefore able to utilize the oscillator to calibrate the field produced by the microwave strip (Fig. 24). We applied zero current through the microwave strip, while varying the DC magnetic field and measuring the oscillator response. We calculated the rate of change of the frequency with respect to the external magnetic field. Similar measurements performed with fixed DC field, but varying currents through the stripline yielded the rate of change of the frequency with respect to the applied DC current. Taking the ratio of these two quantities enabled us to obtain the dependence of the microwave field on the applied current: $h_{e}(\mathrm{Oe})=9.0 i_{a p p}(\mathrm{~mA})$.

The frequency-dependent microwave losses were determined by utilizing a separately fabricated transmission line with the same microstrip geometry. These losses were taken into account when 
determining the microwave Oersted field generated by the microstrip. Loss-adjusted ac currents of up to $4 \mathrm{~mA}$ rms were applied to the microstrip without noticeable heating, producing $h_{e}$ of up to 36 Oe rms at the location of the oscillator.

Microwave signals applied to the microstrip induced parasitic currents in STNO in addition to the Oersted field. In our measurements, the parasitic coupling did not exceed $-25 \mathrm{~dB}$, inducing microwave currents of less than $12 \mu \mathrm{A}$ rms at the largest driving amplitude. As demonstrated below, the effects of these parasitic currents on the oscillation are smaller than those of the microwave field.

\subsubsection{Nanopillars}

Calibration of the Oersted field produced by the microstrip was performed by comparing the dependence of $f_{0}$ on the bias field $H_{0}$ to its dependence on the dc current applied to the microstrip, similar to the method employed for the hystersis investigations. Application of microwave current generated fields of up to $h_{e}=30$ Oe at the location of STNO without noticeable heating. Our technique enabled spectroscopic measurements at arbitrary relations between the driving and the oscillation frequencies, and provided the strong driving force essential for the observation of the phenomena described below. The parasitic coupling between the microstrip and the STNO was less than $-20 \mathrm{~dB}$, resulting in induced microwave currents that did not exceed $40 \mu \mathrm{A}$.

\subsection{Observation of Fractional Synchronization}

\subsubsection{Introduction to Fractional Synchronization}

As the dimensions of electronic devices continue to decrease, higher current densities are required for device operation. These large current densities can drive the system out of equilibrium, generating new phenomena. These non-equilibrium phenomena can serve as a basis for novel device architectures that directly exploit their effects. In particular, large current densities in nanomagnetic devices can result in large-amplitude magnetization oscillations, thus enabling the operation of a novel nanoscale microwave oscillator, the spin-torque nano-oscillator (STNO) $[15,47,48,3]$. Not only is the STNO one of the smallest known auto-oscillating systems, but it is a system that possesses complex nonlinear dynamical properties, whose frequency is dependent on oscillation amplitude, as 

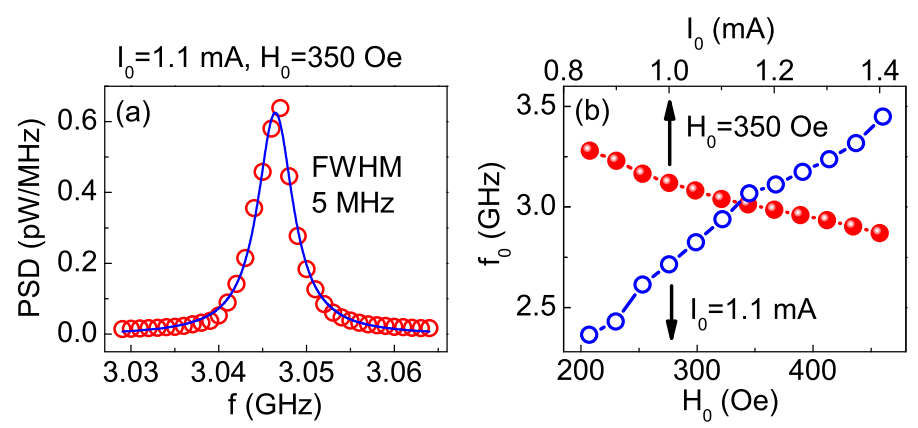

Figure 25: (a) Free-running oscillation characteristics of STNO. Circles: a typical oscillation spectrum. Curve: Lorentzian fit. (b) Oscillation frequency vs bias field (solid blue symbols and bottom scale), and vs bias current (open red symbols and top scale).

a consequence of the dependence of the demagnetizing fields on the oscillation trajectory.

The nonlinearity of the STNO enables the tuning of oscillation frequency by electric current, as well as enhancing its ability to synchronize to periodic external perturbations. This could provide a route for enhancing the oscillation characteristics, by mutual synchronization, in arrays of STNO. Recently, the synchronization of two $[2,49]$ and four STNO [50] with similar frequencies has been demonstrated.

Our approach to synchronization was to utilize a microwave antenna, fabricated directly on top of the STNO device. This is in contrast to the more typical approach of utilizing a driving signal from a microwave current $[51,52,53]$. This approach has enabled us to demonstrate that the complicated nonlinear oscillation characteristics of the STNO can lead to a class of synchronization phenomena which can be observed not only for driving frequency $f_{e}$ close to $f_{0}$, but also when their ratio $r=f_{e} / f_{0}$ is close to integer or certain rational numbers. Theretical analysis will be shown which will show that these phenomena provide valuable information about the oscillation properties.

\subsubsection{Device Characterization}

Characterization of STNO was performed by measurements of auto-oscillation spectra. The bias field was $H_{0}=350$ Oe unless specified otherwise, applied in-plane perpendicular to the microstrip. All measurements were performed at $5 \mathrm{~K}$. The measured peaks exhibited a typical full width at half maximum (FWHM) of $5 \mathrm{MHz}$ and Lorentzian line shape characteristic of thermal broadening 


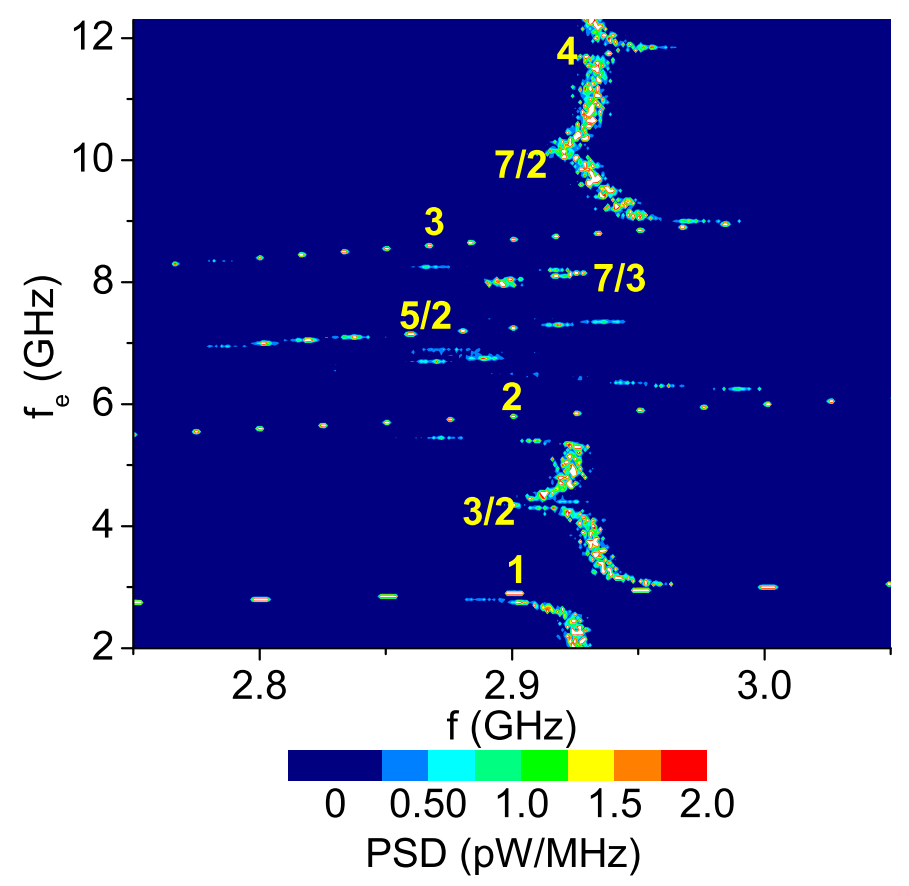

Figure 26: Dependence of the oscillation spectra on the driving frequency, at $h_{e}=13$ Oe and $I_{0}=1.3 \mathrm{~mA}$. The static magnetic field $H=350 \mathrm{Oe}$. The scale indicates the power spectral density (PSD) of the spectral signal. The values of $r=f_{e} / f_{0}^{\prime}$ for the identified synchronization regimes are labeled. $\Theta=20^{\circ}$ as defined in 20 (a).

(Fig. 25 (a)) $[54,55]$. Thermal broadening is an example of homoegenous broadening and is a consequence of the fact that the local magnetic moments in the active device area all have equal probability of fluctuating. The oscillation frequency increased with increasing $H_{0}$, and decreased with increasing $I_{0}$ (Fig. 25 (b)), consistent with the established properties of similar STNOs [3]. The reported behaviors were confirmed for three devices.

\subsubsection{Experimental Results and Discussion}

In spectroscopic measurements of oscillation at finite driving field $h_{e}$, the oscillation frequency $f_{0}^{\prime}$ remained close to the free-running frequency $f_{0}=2.94 \mathrm{GHz}$ for most of the values of the driving frequency $f_{e}$, with the exception of the regions where the ratio $r=f_{e} / f_{0}$ is close to all integer $(r=$ $1,2,3,4)$ and several rational $(r=3 / 2,7 / 3,5 / 2,7 / 2)$ values (Fig. 26). In these regions, $f_{0}^{\prime}$ follows a linear relationship with $f_{e}$, as expected for the synchronized oscillation. Since the spectroscopic 

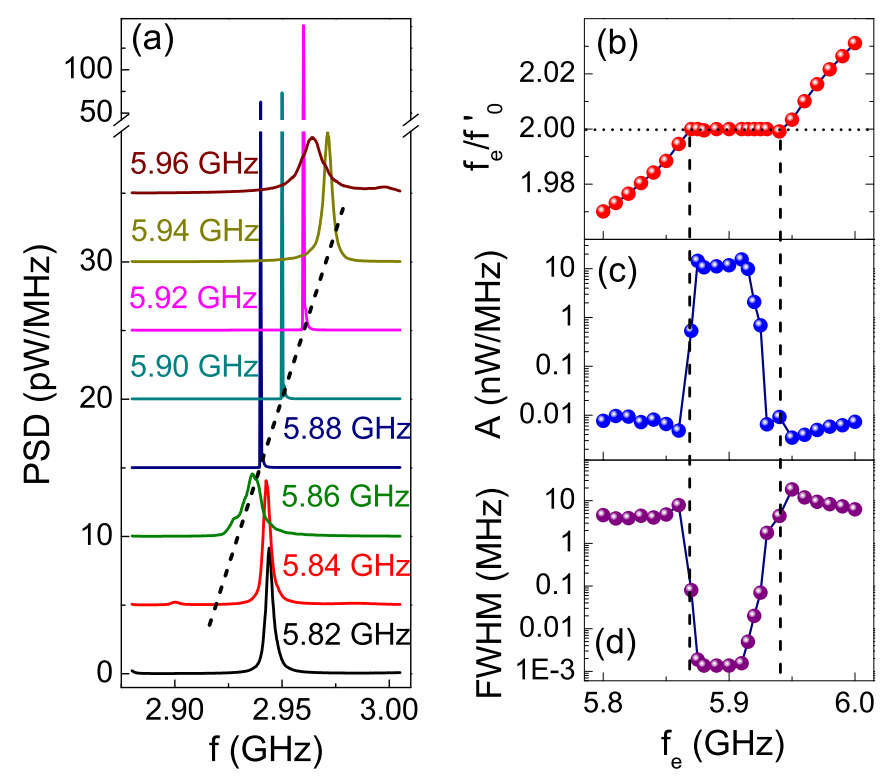

Figure 27: The $r=2$ synchronization at $I_{0}=1.3 \mathrm{~mA}, h_{e}=2$ Oe. (a) Oscillation spectra at the labeled values of $f_{e}$, acquired with a bandpass of $300 \mathrm{kHz}$. Dotted line indicates $f=f_{e} / 2$. (b)-(d) Characteristics of the oscillation peaks acquired with a bandpass of $1 \mathrm{kHz}$ : (c) the ratio of $f_{e}$ to $f_{0}^{\prime}$, (c) the peak spectral amplitude $A$, (d) FWHM of the oscillation peak. Dashed lines show the synchronization boundaries.

peaks observed at $r=1$ are dominated by the parasitic coupling to the driving signal, in this regime the synchronization can be identified by the breaks in the $f_{0}^{\prime} \approx$ const line. In contrast, spectroscopic measurements of all the other synchronization regimes were performed below the driving frequency, eliminating the effects of parasitic signals on the spectra, and enabling us to directly determine the characteristics of the synchronized oscillations.

An example of spectroscopic measurements for one of these regimes $(r=2)$ is shown in Fig. 27 (a). The synchronized oscillation peaks become significantly narrower and their maximum spectral amplitude dramatically increases, while the oscillation frequency follows an exact relationship $f_{0}^{\prime} / f_{e}=2$ within the measurement precision of better than $0.05 \%$ (Fig. 27 (b)). To enable a wide frequency scan shown in Fig. 27 (a)), these measurements were performed with a bandpass of $300 \mathrm{kHz}$ that exceeded the width of the synchronized peak. Separate measurements at our setup's ultimate resolution of $1 \mathrm{kHz}$ showed that synchronization resulted in an increase of the maximum spectral amplitude by three orders of magiutude Fig. 27(c), and a similar magnitude of decrease of FWHM to the smallest value of $1.4 \mathrm{kHz}(\mathrm{Fig} .27(\mathrm{~d}))$. These results demonstrate that the oscillator becomes 

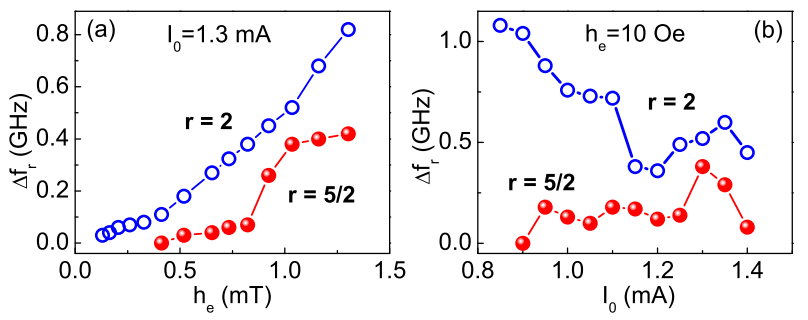

Figure 28: (a) Dependence of the integer synchronization interval $\Delta f_{2}$ (open symbols) and fractional interval $\Delta f_{5 / 2}$ (solid symbols) on $h_{e}$. (b) Dependence on $I_{0}$.

fully phase-locked with the driving signal for at least $1 \mathrm{~ms}$ (3 million oscillation cycles) without a single phase slip. We note that the total power generated by STNO is determined by the amplitude of the magnetization oscillation, which is not significantly affected by the driving field. Indeed, the increase of the peak amplitude is compensated by the decrease of the linewidth, so that the total generated power remains approximately constant.

To quantitatively characterize the synchronization, we define the synchronization interval $\Delta f_{r}=$ $\left(f_{e, \max }-f_{e, \min }\right) / r$, where $f_{e, \max }\left(f_{e, \min }\right)$ is the maximum (minimum) driving frequency at which the synchronization is observed. Although both the integer interval $\Delta f_{2}$ and the fractional interval $\Delta f_{5 / 2}$ increase with the driving amplitude, the value of $\Delta f_{2}$ depends linearly on $h_{e}$, while the dependence of $\Delta f_{5 / 2}$ on $h_{e}$ is strongly nonlinear (Fig. 28 (a)). Integer synchronization persisted at the smallest driving signal $h_{e}=1.3$ Oe used in our measurements, while the $r=5 / 2$ synchronization disappeared at $h_{e}<4$ Oe. The value of $\Delta f_{2}$ increases at small $I_{0}$, exceeding $1 \mathrm{GHz}$ at $I_{0}<0.9 \mathrm{~mA}$. In contrast, $\Delta f_{5 / 2}$ decreases at small $I_{0}$, vanishing at $I_{0}<0.9 \mathrm{~mA}$ (Fig. 28 (b)).

\subsubsection{Dependence of Spectra on Current and Magnetic Field}

Measurements of fractional synchronization acquired at different combinations of fields, currents, and orientations can be observed in Figs. 29 and 30. Examination of these spectra reveals an intricate dependence of the fractional synchronization regimes on the magnitude and direction of applied field.

Spectra acquired at $I_{0}=1.3 \mathrm{~mA}$ and $H=540$ Oe (Fig. 29) are markedly different from data acquired with the same DC bias current and $H=350$ Oe. The most notable difference is the obser- 


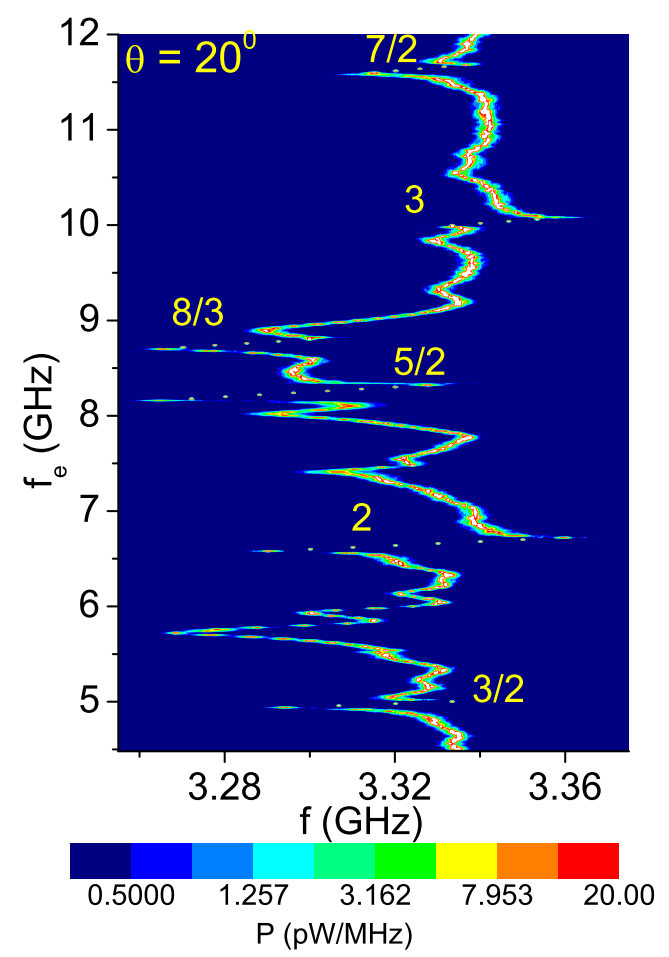

Figure 29: Dependence of the oscillation spectra on the driving frequency, at $h_{e}=13$ Oe and $I_{0}=1.3 \mathrm{~mA}$ with $H=540 \mathrm{Oe}$. The scale indicates the power spectral density (PSD) of the spectral signal. The values of $r=f_{e} / f_{0}^{\prime}$ for the identified synchronization regimes are labeled.

vation of strong frequency pulling in the unsynchronized regime. In addition, the nominally unentrained state is characterized by a clear dependence of $f$ on the driving frequency. This dependence is somewhat remarkable, as none of the other acquired spectra demonstrate such a phenomenon.

Reducing the driving current to $I_{0}=1.0 \mathrm{~mA}$ and static magnetic field $H=380$ Oe result in a spectra (Fig. 30 (b)) which is qualitatively different than measurements performed at higher current. This regime is characterized by regions in frequency space in which the STNO does not oscillate. In particular, between the fundamental and second harmonic there is a large region in which there is no oscillation, as well as between the $5 / 2$ and 3 synchronized states. We also note that in this regime there is significantly smaller frequency pulling than what is seen in Fig. 29.

Rotation of the field by $90^{\circ}$ with respect to the nanopillar easy axis, keeping the magnitude of the applied field and current constant, results in a remarkable change in the oscillation characteristics (Fig. 30 (a)). We again observe a large degree of frequency pulling as the oscillator approaches the 


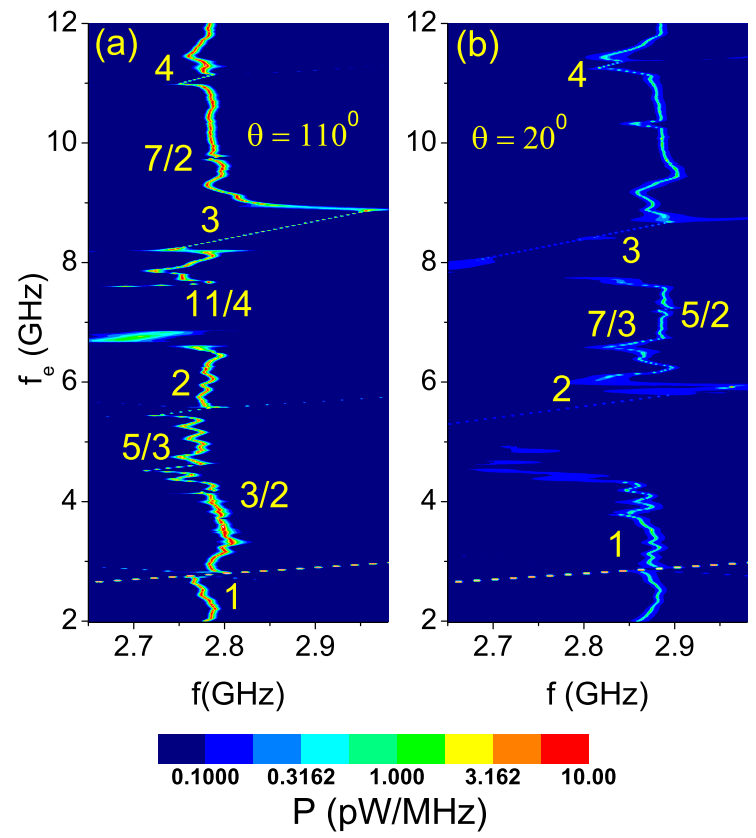

Figure 30: Dependence of the oscillation spectra on the driving frequency, at $h_{e}=13$ Oe and $I_{0}=1.0 \mathrm{~mA}$ with $H=380 O e$. (a) Spectra acquired at $\theta=110^{\circ}$ (b) Spectra for $\theta=20^{\circ}$. The scale indicates the power spectral density (PSD) of the spectral signal. The values of $r=f_{e} / f_{0}^{\prime}$ for the identified synchronization regimes are labeled.

locked state, as well as oscillations in the unsynchronized states having linewidths on the order of GHz and small amplitude. We observe a number of fractional regimes, including $3 / 2,5 / 3,11 / 4$, and 7/2. In this orientation, there are more fractional regimes than what is observed in Fig. 30 (b).

\subsubsection{Theoretical Model of Fractional Synchronization}

The complexity of the observed synchronization patterns suggests that they contain intricate information about the oscillator. To analyze the mechanisms of synchronization, we consider the classic model describing the state of the oscillator only by the phase $\phi$ defined to increase linearly with time in the autonomousregime $[56,57]$. Under the influence of a periodic driving signal, the dynamics can be described by

$$
d \phi / d t=\omega_{0}+\mu R e\left[g(\phi) e^{-i \omega_{e} t}\right]
$$


Here, $\mu$ is the amplitude of the driving force, $\omega_{0, e}=2 \pi f_{0, e}$, and the complex function $g(\phi)$ determined by the geometry of the oscillation trajectory and the driving signal describes the position-dependent sensitivity of the oscillator to the driving force. Since $g(\phi)$ is periodic, it can be expressed as $g(\phi)=\sum_{n} g_{n} e^{i n \phi}$, where the Fourier components $g_{n}$ contain the information about the oscillation trajectory.

To determine the relation of the function $g(\phi)$ to synchronization, we consider a fractional synchronized state $r=p / q$, where $p$ and $q$ are mutually simple integers. The oscillator completes $q$ cycles during $p$ periods of the driving force, i.e. its phase satisfies $\phi\left(t+p / f_{e}\right)=\phi(t)+2 \pi q$. Expanding it into a Fourier series, $\phi(t)=\phi(0)+\omega_{e} t q / p+\sum_{k \neq 0} \phi_{k} e^{-i \omega_{e} t / p}$ and inserting into equation (3.1), we obtain an equation for $\phi_{k}$ which can be solved iteratively in powers of $\mu$. The condition of solvability of this equation determines the synchronization interval $\Delta \omega_{r}$.

To the first order in $\mu$, only the synchronization at integer $r=p / 1$ is possible, with the interval

$$
\Delta \omega_{p} \approx 2 \mu\left|g_{p}\right|
$$

Fractional synchronization $(q>1)$ appears only in the higher orders in $\mu$, with intervals $\Delta \omega_{r} \propto$ $\left(\mu g_{p}\right)^{q}$. In particular, for $r=p / 2$

$$
\Delta \omega_{p / 2} \approx \frac{\mu^{2}}{\omega_{0}}\left|\sum_{n} g_{n} g_{p-n}\right|
$$

The linear dependence of $\Delta \omega_{p}$ on $\mu \propto h_{e}$, and the nonlinear dependence of $\Delta \omega_{p / 2}$ are in agreement with Fig. 28 (a).

It is generally difficult to calculate the function $g(\phi)$ that determines the synchronization intervals, since it depends both on the form of the driving signal and the oscillation trajectory. However, it is possible to experimentally determine each component $g_{p}$ by measuring the corresponding synchronization intervals(Eq. 3.2). Moreover, symmetry analysis described below enables one to determine the general conditions for the existence of specific synchronization regimes, providing a qualitative insight into the mechanism of fractional synchronization.

The oscillation trajectory of STNO is approximately symmetric with respect to a half period rotation $\phi \rightarrow \phi+\pi[3]$. A microwave field parallel to the oscillation symmetry axis $\mathbf{s}$ produces a 
symmetric driving force, i.e. a $\pi$ phase-shift of the driving signal does not change the oscillation phase. In this case, all the odd Fourier components of $g(\phi)$ vanish, $g_{2 n+1}=0$, and only even integer synchronization regimes can be observed. On the other hand, $\mathbf{h}_{e} \perp \mathbf{s}$ produces an antisymmetric driving force. In this case, all the even components of $g(\phi)$ vanish, $g_{2 n}=0$, and only odd integer synchronization regimes can be observed.

To understand the implications of our symmetry analysis for fractional synchronization, we consider the half-integer regime (Eq. (3.3)). Since the numbers $p$ and $q=2$ are mutually simple, $p$ is odd. Therefore, the indices of the Fourier components $g_{n}$ and $g_{p-n}$ in equation (3.3) have different parity. Since the even spectral components describe perturbations that are symmetric with respect to a half-period rotation, and the odd ones describe anti-symmetric perturbations, the $q=2$ fractional synchronization of a symmetric auto-oscillator is possible only if the driving signal contains both symmetric and anti-symmetric components, or in other words, if the signal breaks the symmetry of the oscillator. In our experiment, $\mathbf{h}_{e}$ was parallel to $\mathbf{H}_{0}$, at $45^{\circ}$ with respect to the nanopillar axis. Modeling shows that, as a result of the demagnetizing effects, $\mathbf{s}$ was rotated by $20^{\circ}$ with respect to $\mathbf{h}_{e}$, creating the symmetry-breaking geometry necessary for the fractional synchronization.

To confirm the results of our analysis, we performed numerical simulations of STNO synchronization to a microwave field (Fig. 31). The temporal evolution of magnetization was determined by integration of the Landau-Lifshitz equation within a macrospin approximation, which included the spin-torque and the driving field terms, with the parameter values corresponding to the experimental conditions of Fig. $26 H_{0}=350$ Oe oriented at $45^{\circ}$ with the nanopillar easy axis, $I_{0}=1.3 \mathrm{~mA}$, $h_{e}=13$ Oe, saturation magnetization $M_{s}=640 \mathrm{G}$, easy-axis anisotropy field $H_{a}=200$ Oe, Gilbert damping constant $\alpha=0.01$ [58], and dimensionless spin-polarization efficiency $\eta=0.18$ [15]. The spectral characteristics of the driven oscillation were determined by Fourier transformation of the simulated dynamics.

In agreement with our symmetry analysis, only the even integer synchronization regimes are prominent for $\mathbf{h}_{e} \| \mathbf{s}$ (Fig. 31(a)), and only the odd integer synchronization regimes are prominent for $\mathbf{h}_{e} \perp \mathbf{s}$ (Fig. 31(b)). In both cases, small but finite fractional synchronization ranges are caused by the slight asymmetry of the oscillation trajectory. On the other hand, simulation of the experimental configuration $\mathbf{h}_{e} \| \mathbf{H}_{0}$ (Fig. 31(c)), corresponding to an angle of $20^{\circ}$ between $\mathbf{h}_{e}$ and s, produces 


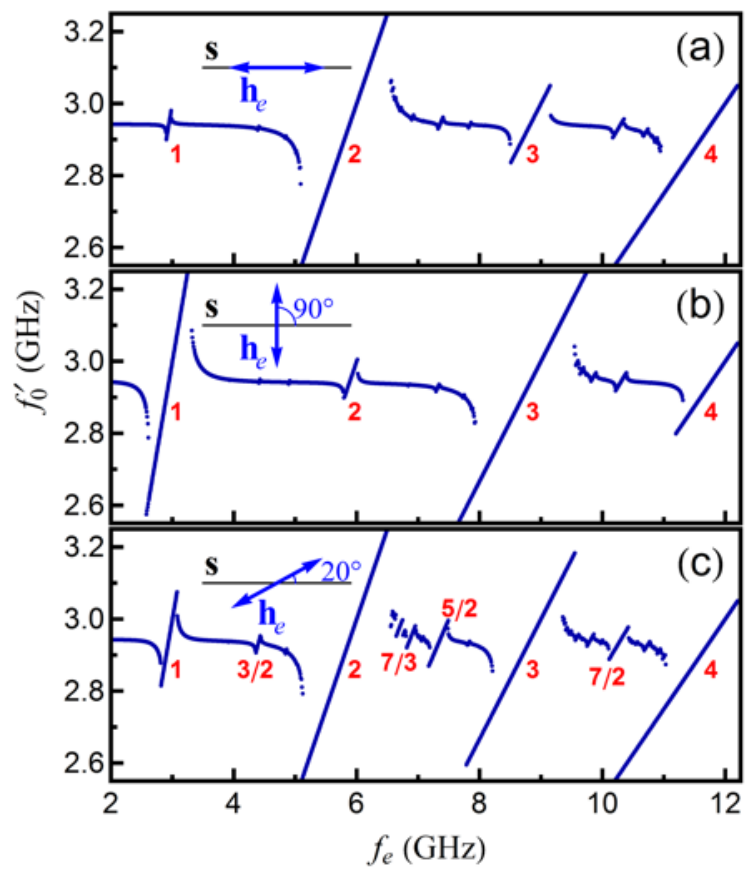

Figure 31: Simulated dependence of $f_{0}^{\prime}$ on $f_{e}$, for different orientations of $\mathbf{h}_{e}$ with respect to $\mathbf{s}$ : (a) $\mathbf{h}_{e} \| \mathbf{s}$, (b) $\mathbf{h}_{e} \perp \mathbf{s}$, and (c) angle of $20^{\circ}$ between $\mathbf{h}_{e}$ and $\mathbf{s}$ corresponding to the experimental configuration. Prominent synchronization regimes are labeled.

large integer andfractional synchronization ranges, in excellent semi-quantitative agreement with the data (Fig. 26). Additional simulations for $f_{e}<f_{0}$ showed that fractional synchronization tosymmetry-breaking perturbations is also possible at $r<1$.

Our symmetry analysis is equally applicable to synchronization by microwave current. In this case, the symmetry of the driving force is determined by the direction of the current polarization. In the published measurements of the main synchronization regime $r=1[51,52,53]$ the current polarization was nearly parallel to $\mathbf{s}$, and consequently the driving force was approximately symmetric. Since synchronization with $r=1$ requires anti-symmetric perturbation (Eq. (3.2)), our analysis explains why a large magnitude of the driving current comparable to $I_{0}$ was required in these measurements. The anti-symmetric perturbation produces a synchronization interval that is proportional to the square of the oscillation amptlitude. Therefore, for small oscillations the locking interval would be small. In contrast, a significantly more efficient $r=2$ synchronization can be expected in this geometry, due to the dependence of the synchronization interval on the square of the oscillation amplitude. 


\subsubsection{Conclusions}

In conclusion, we have experimentally demonstrated synchronization of a spin torque nano-oscillator at fractional ratios between the frequency of the driving microwave field and the frequency of the oscillation. The phenomenon of fractional synchronization opens new routes for efficient synchronization of auto-oscillators whose frequencies are not close to each other, and for the development of novel nano-scale signal processing devices such as microwave frequency converters. We developed a general model of synchronization, which shows that fractionalsynchronization of symmetric oscillators becomes possible only if the driving force breaks the oscillation symmetry, and more generally, that the efficiency of synchronization in any regime is determined by the symmetry of the driving signal. Fractional synchronization for a controlled symmetry of the driving signal represents a novel tool for the characterization of auto-oscillators. For instance, one can determine the orientation of the precession axis by measuring the dependence of the synchronization intervals on the direction of the driving field.

\subsection{Observation of Synchronization Hysteresis}

\subsubsection{Introduction to Hysteretic Synchronization}

Among the auto-oscillating systems, the class of nonlinear auto-oscillators is characterized by a strong dependence of their oscillation frequency $\omega_{0}$ on the power $p$ (or amplitude) of the oscillation $[56,59,60]$. When such a system is perturbed by a periodic force of sufficient strength, its nonlinearity can enhance the range of synchronization. This effect results from the reduction of the detuning between $\omega_{0}$ and the frequency $\omega_{e}$ of the driving force, caused by the variations of $p[56,60]$.

The nonlinearity can also have a qualitative effect on the dynamical properties of the oscillator. Novel phenomena can arise as a consequence of the nonlinearity, which would not be seen in linear systems. In quasi-linear auto-oscillating systems, the time evolution of the difference in phase between the oscillator and driving force, and hence synchronization can be described by Adler's equation [56, 57], and the synchronization transition is interpreted as the simultaneous creation of a stable node and a saddle point, which is a non-hysteretic process. In contrast, for nonlinear oscillators, such as magnetic spin-torque nano-oscillators (STNO) $[15,47,48,3]$, analysis that in- 
cludes variations of both the phase and the amplitude indicates that periodic and quasi-periodic states can remain simultaneously stable, resulting in hysteretic synchronization [61]. This predicted, but previously unobserved, effect is qualitatively different from the well-known hysteretic transition between different synchronization regimes [56], which is caused by the coexistence of multiple stable synchronized states and does not require nonlinearity.

We have achieved hysteretic synchronization of a point contact spin torque nano-oscillator to an external periodic perturbation, provided by a microwave magnetic field. This hysteresis has an onset that correlates with a region of a strong dependence of the auto-oscillation frequency on the bias current, suggesting a correlation of this phenomenon with the fundamental nonlinear properties of the oscillator. Contrary to the theoretical analysis which predicted that hysteresis would be symmetric with respect to the sign of detuning [61], we have observed hysteresis only at the lower or upper synchronization range, i.e. the hysteresis is asymmetric.

\subsubsection{Device Characterization}

The static and dynamical magnetic characterics of the spin torque oscillator were determined by measurements of giant magneto-resistance and auto-oscillation spectra, respectively. All the measurements were performed at $5 \mathrm{~K}$, at a dc bias field $H_{0}=1.1 \mathrm{kOe}$. The reported results were confirmed for two devices. Both devices exhibited clear hysteretic switching behavior at low DC magnetic fields, with a giant magneto-resistance of approximately $2 \% 32$.

The auto-oscillation spectra had a Lorenzian lineshape with a typical full width at half-maximum of $4 \mathrm{MHz}$, consistent with the effects of thermal broadening [54, 55, 60](Fig. 33 (b)). Above the oscillation onset at bias current $I_{t}=2.0 \mathrm{~mA}$, the frequency $f_{0}$ of the auto-oscillation was initially approximately constant. However, for $I_{0}>2.75 \mathrm{~mA}$, it exhibited a region of strong red-shifting with a decreasing slope $d f_{0} / d I_{0}$ (Fig. $33(\mathrm{c})$ ). Comparison of the dependence of the auto-oscillation frequency on bias current with the dependence of the emitted power on bias current reveals that this red shifting region is correlated with the increase of the slope in the dependence of the emitted power on the bias current (Fig. 33 (d)). This behavior is expected for a nonlinear oscillator, as the output power is proportional to the amplitude of oscillation. As the oscillation amplitude increases, the frequency should correspondingly decrease. In a linear oscillator, the frequency is independent 


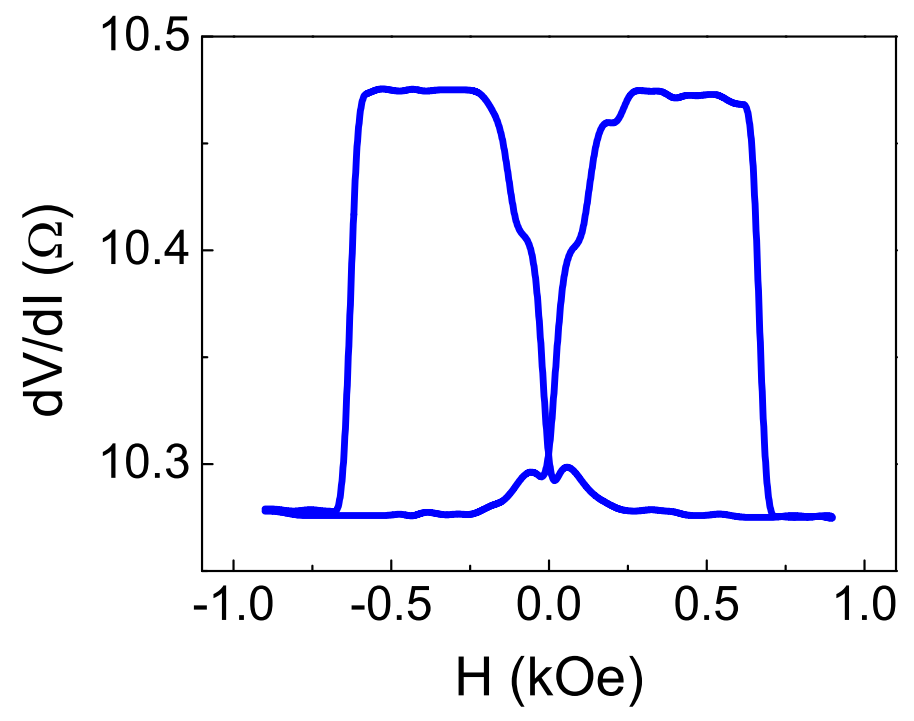

Figure 32: Differential resistance curve for the point contact device.

(a)
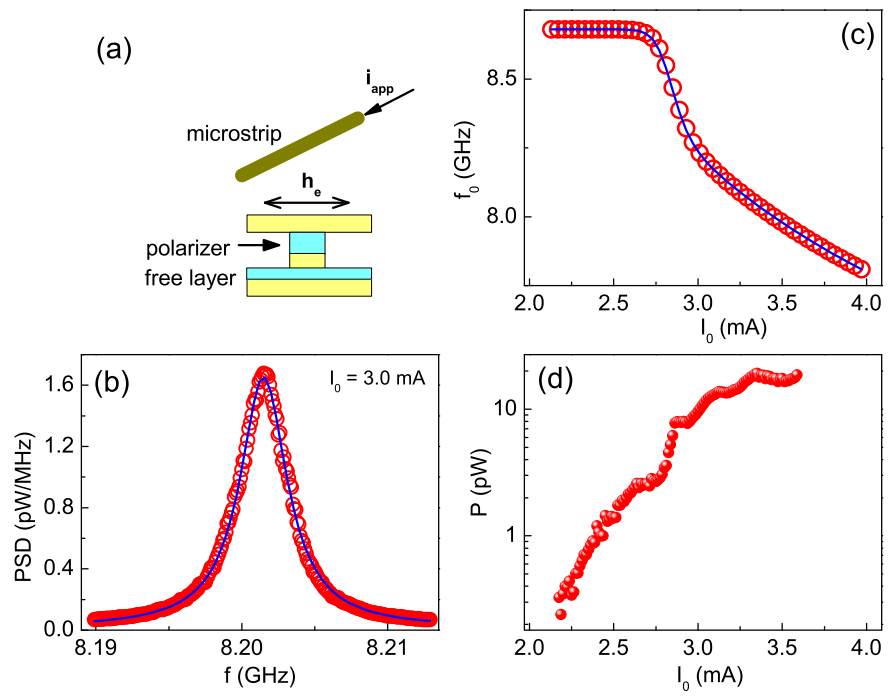

Figure 33: (a) Schematic of the device including an STNO and a microstrip line generating a synchronizing Oersted field. (b) Example of auto-oscillation spectrum at $I_{0}=3 \mathrm{~mA}$ (symbols), and Lorentzian fitting with full width at half maximum of $4 \mathrm{MHz}$ (solid curve). (c) Auto-oscillation frequency vs bias current (red open circles). Blue curve is the fit from Eqn. 3.5. (d) Emitted power under the oscillation peak vs bias current. 


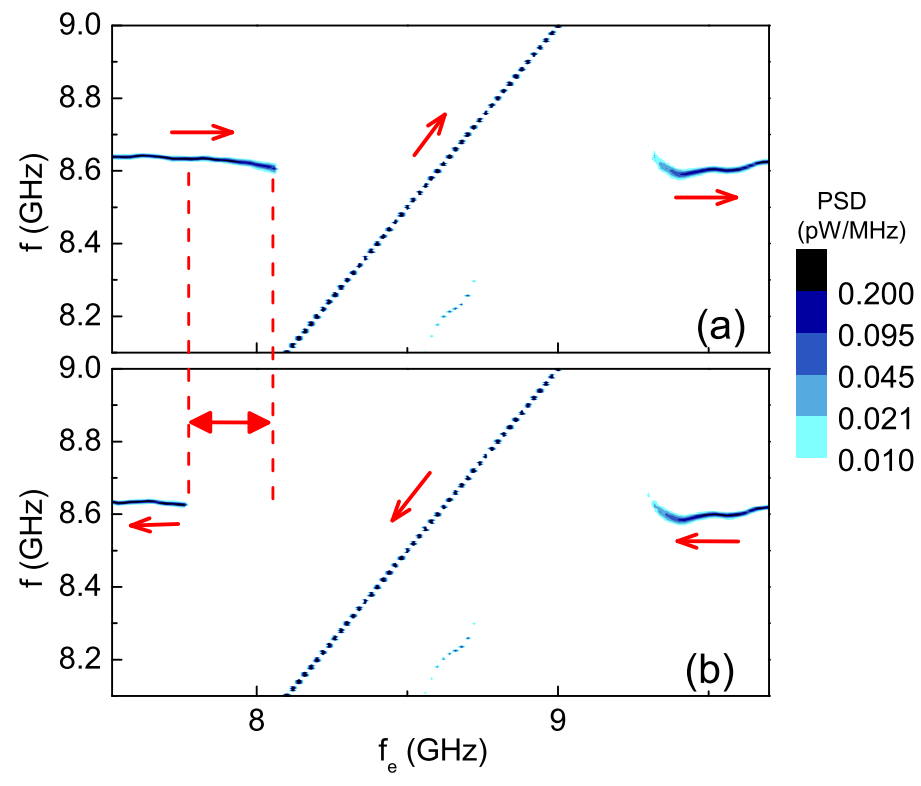

Figure 34: Dependence of the oscillation frequency $f$ of STNO on the driving frequency $f_{e}$, demonstrating hysteretic synchronization to the external signal: (a) increasing $f_{e}$ and (b) decreasing $f_{e}$, at $I_{0}=2.7 \mathrm{~mA}$. The bias field $H_{0}$ is perpendicular to the microwave driving field $h_{e}=18$ Oe. Arrows show the direction of the scan, and dashed vertical lines show the limits of the hysteretic synchronization range; a double arrow shows the width of the hysteresis interval.

of the amplitude of oscillation, and such a correlation would not be observed. In both samples, hysteresis was observed in the vicinity of this strong frequency red shift.

A possible source of experimental error in our setup is the presence of parasitic coupling. This occurs when circuit elements, which have an internal capacitance, are exposed to high frequency AC signals. Parasitic coupling produced peaks at $f=f_{e}$ in spectroscopic measurements of driven oscillations, regardless of the oscillation regime. Therefore, the transition to the synchronized regime was identified as an abrupt disappearance of the $f_{0} \approx$ const line of unlocked oscillation (Fig. 34).

One general feature of synchronization in linear oscillators is frequency pulling. This occurs before the transition to the synchronized regime, and is characterized by an oscillator frequency which is neither equal to the perturbation, or the oscillator's natural frequency. In our spectroscopic data, this would manifest itself as a curved feature in the oscillator response. We note that frequency pulling is negligible in the data of Fig. 34, i.e. the transition is abrupt rather than continuous.

The hysteresis is demonstrated by the difference in the synchronization transition depending on 

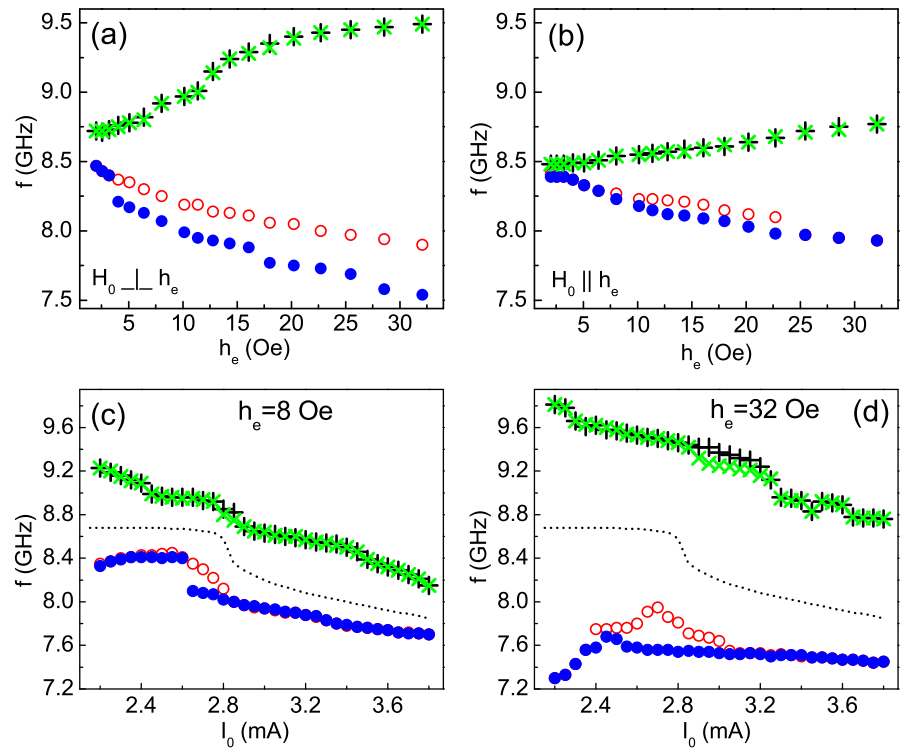

Figure 35: (a) Dependence of the synchronization boundaries on the rms amplitude $h_{e}$ of the driving microwave magnetic field, for $H_{0} \perp h_{e}$ at $I_{0}=2.7 \mathrm{~mA}$. Black crosses: upper synchronization boundary for $f_{e}$ scanned up, green X: upper synchronization boundary for $f_{e}$ scanned down, red open circles: lower synchronization boundary for $f_{e}$ scanned up, blue solid circles: lower synchronization boundary for $f_{e}$ scanned down. (b) Same as (a) for $H_{0} \| h_{e}$ at $I_{0}=2.7 \mathrm{~mA}$. (c) Dependence of the synchronization boundaries on the bias current $I_{0}$, at $h_{e}=8$ Oe perpendicular to $H_{0}$. Dashed curves show the auto-oscillation frequency. (d) Same as (c) for $h_{e}=32$ Oe perpendicular to $H_{0}$.

the direction of the driving frequency sweep. The frequency at the lower boundary of the detuning interval in the synchronized regime is larger by $0.31 \mathrm{GHz}$ for $f_{e}$ scanned up (Fig. 34(a)) than for $f_{e}$ scanned down (Fig. 34(b)). We note that the frequency of the upper synchronization boundary is the same for both directions of driving frequency sweep.

In addition to the phenomenon of hystersis, an additional observation can be made regarding the data in Fig. 34. In the entrained state, there are additional peaks in the spectra with a nonlinear dependence on the frequency of the applied microwaves. This data is the same, in both directions of the driving field sweep. We believe this phenomenon is a consequence of mixing between the fundamental and higher-order oscillation modes. The nature of these other modes was not studied in this work and remains unknown, but is worthy of future study. 


\subsubsection{Experimental Results and Discussion}

The synchronization range and hysteresis both exhibited a strong dependence on experimental geometry. They were significantly larger for $H_{0} \perp h_{e}$ than for $H_{0} \| h_{e}$, confirming the dominance of the effects of the microwave field over the parasitically induced microwave current (Fig. 35 (a) and, 35 (b)). If the parasitic currents played a significant role in the spectroscopic response, we would expect that the synchronization and hysteresis would be comparable for both experimental geometries. A similar dependence of hysteresis on the field orientation was also observed in the other tested device. These data explain why hysteresis has not been previously observed in measurements of synchronization induced by a microwave current.

Comparison of measurements of the dependence of synchronization on $I_{0}$ with the auto-oscillation spectra indicate that the emergence of hysteresis is correlated with the nonlinear properties of the auto-oscillator. At a small driving field $h_{e}=8$ Oe (Fig. 35 (c)), the hysteresis appears at the lower boundary of the synchronization range at $2.65 \mathrm{~mA} \leq I_{0} \leq 2.8 \mathrm{~mA}$, i.e. close to the region where the auto-oscillation (dashed curve) exhibits the largest frequency red shift. A small hysteresis is also observed at the upper boundary of the synchronization range at $I_{0}=2.8 \mathrm{~mA}$ to $2.85 \mathrm{~mA}$.

In a typical geometry of spin transfer devices, the current polarization is nearly collinear with the static magnetization of the free layer. In this case, the synchronization effects are similar to those of $h_{e} \| H_{0}$ in Fig. 35 (d), producing only a small hysteresis. Moreover, our data show that there is a threshold microwave field for the appearance of hysteresis(Figs. 35(a), and 35(b)). It is likely that the typical experimental geometry would not enable the application of sufficiently strong driving signal to induce hysteresis. A driving microwave current with polarization perpendicular to the magnetization of the free layer would likely produce a large hysteresis, similar to our geometry with $h_{e} \perp H_{0}$. However, such a geometry would not allow excitation of auto-oscillations via the spin transfer effect [62].

At a larger driving amplitude $h_{e}=32$ Oe (Fig. 35 (d)), the synchronization interval and the magnitude of hysteresis increase, and hysteretic synchronization is observed over a larger range of bias currents. The hysteresis at the upper boundary of synchronization interval remains small, but occurs over a broad range of $I_{0}$ from $2.85 \mathrm{~mA}$ to $3.2 \mathrm{~mA}$. The observed asymmetry of hysteresis was confirmed in both tested devices for several values of the bias field. 


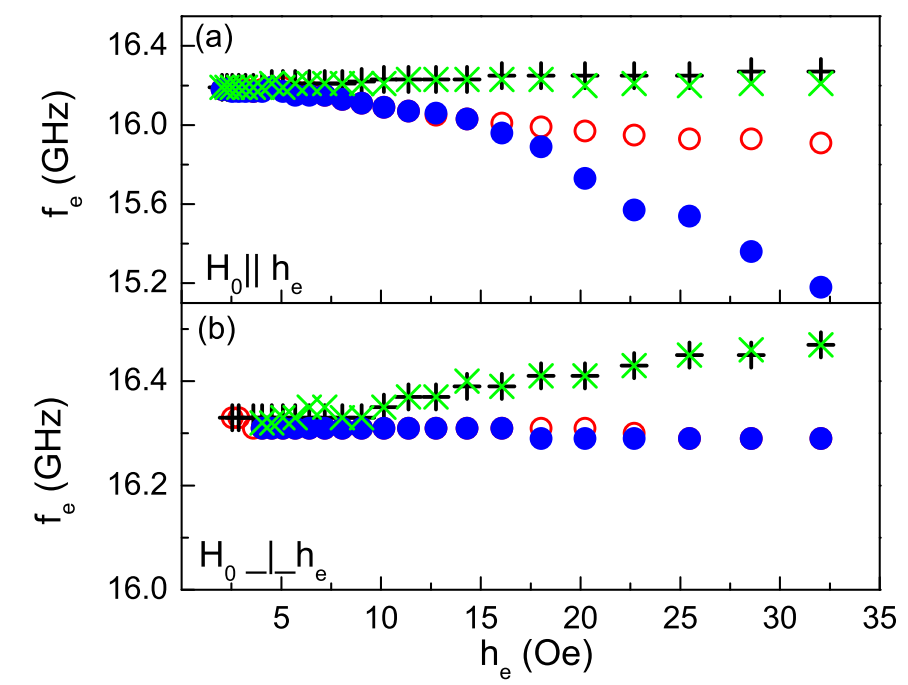

Figure 36: (a) Dependence of the parametric synchronization boundaries on the rms amplitude $h_{e}$ of the driving microwave magnetic field, for $H_{0}=1 k O e \| h_{e}$ at $I_{0}=3.1 \mathrm{~mA}$. Black crosses: upper synchronization boundary for $f_{e}$ scanned up, green X: upper synchronization boundary for $f_{e}$ scanned down, red open circles: lower synchronization boundary for $f_{e}$ scanned up, blue solid circles: lower synchronization boundary for $f_{e}$ scanned down. (b) Same as (a) for $H_{0}=1 k O e \perp h_{e}$ at $I_{0}=3.1 \mathrm{~mA}$.

\subsubsection{Parametric Hysteresis}

Measurements were also performed with $f_{e} \approx 2 f_{0}$ to ascertain if parametric synchronization was possible in this geometry. Our measurements demonstrated that it is possible to synchronize in the parametric regime, with the efficiency of synchronization depending on the measurement geometry. We observed that the geometric dependence is complimentary to the hysteresis in the fundamental regime, i.e. the parametric hysteresis is strongest in the $H_{0} \| h_{e}$ geometry (Fig. 36 (a)), for which it is weakest when $f_{e} \approx f_{0}$ (Fig. 35 (b)). Conversely, in the parametric regime the $H_{0} \perp h_{e}$ (Fig. 36 (b)) geometry induces a small hysteresis, similar to the case of $H_{0} \| h_{e}$ in the fundamental regime.

In the $H_{0} \| h_{e}$ geometry, for each value of $h_{e}$ the parametric hysteresis was significantly larger than the corresponding field for the hysteresis in the fundamental frequency (compare Figs. 35, and 36). This can be interpreted in the framework of the theoretical analysis of fractional synchronization. Recall that the synchronization interval will be proportional to amplitude in the fundamental regime, and proportional to the square of the amplitude in the parametric regime. For current $I=3.1 \mathrm{~mA}$ above the transition the oscillation amplitude was quite large, hence the 
synchronization in the parametric regime was more efficient than in the fundamental regime.

One other curious fact is that the hysteresis interval was a monotonic function of the applied microwave field, in contrast to the fundamental regime. Synchronization measurements at the first harmonic yielded a hysteresis interval that would appear at a threshold field, grow in magnitude as $h_{e}$ is increased, then begin to decrease until it became vanishingly small. In the parametric regime, the hystersis interval grew over the entire range of applied fields. We were unable to increase $h_{e}$, due to the substantial risk of damage to the device.
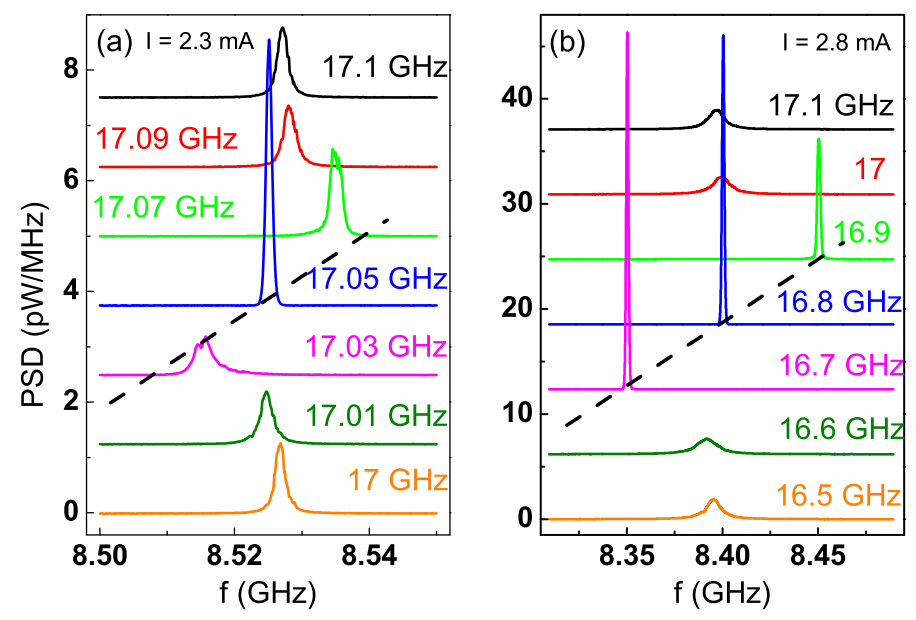

Figure 37: Oscillation spectra for STNO driven above and below twice the auto-oscillation frequency, with $\mathrm{h}_{e}=2$ Oe. (a) $\mathrm{I}_{d c}=2.3 \mathrm{~mA}$, below the transition to the red-shifting regime. (b) $\mathrm{I}_{d c}=2.8$ $\mathrm{mA}$, just above the transition to the red-shifting regime.

Analysis of the oscillation spectra as the STNO is driven close to the parametric regime revealed a strong dependence of the oscillation characteristics on the driving current. For current $(I=2.3 m A)$ below the red-shifting transition, the synchronization results in a strong reduction of the linewidth and a significant change in the profile spectra (Fig. 37 (a)). The full width at half maximum decreased by over five orders of magnitude (Fig. 38 b), with a corresponding modest increase in oscillation power (Fig. 38 (c)). We observed that, even in the synchronized state, there is not a guarantee of a reduction in the oscillation linewidth. In fact, the reduction in linewidth only appears well into the synchronized state, suggesting that the weak nonlinearity in this current regime is inefficient in improving the oscillation characteristics of the STNO. 

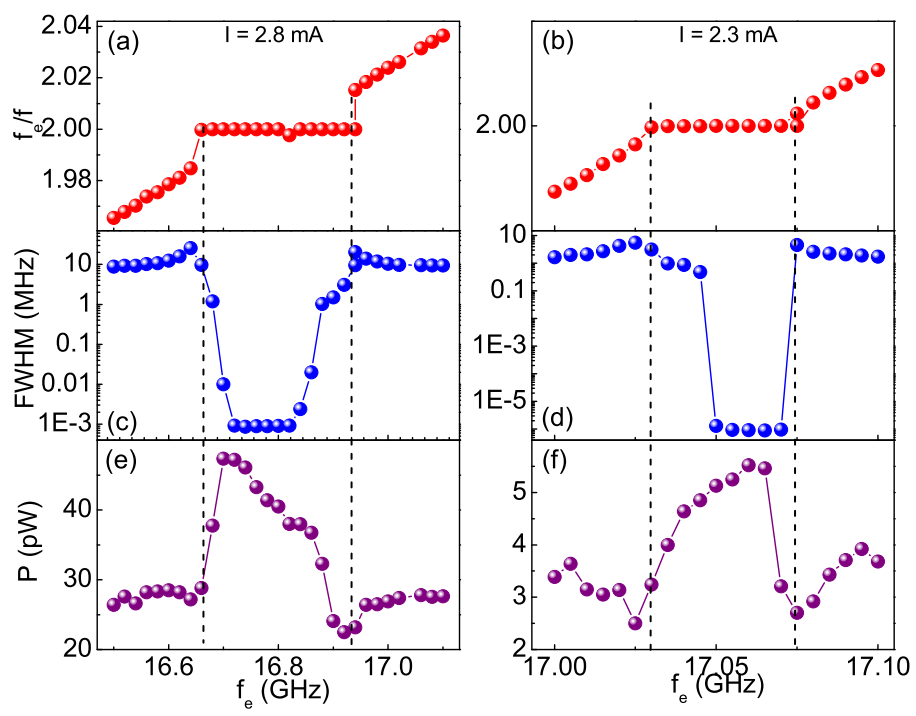

Figure 38: Oscillation properties of the driven STNO with $\mathrm{h}_{e}=2$ Oe and labeled DC current. (a) Ratio of perturbing frequency $\mathrm{f}_{e}$ to oscillation frequency $\mathrm{f}$ with $I=2.8 \mathrm{~mA}$. (b) Full width at half maximum (FWHM) of the oscillation. (c) Total power under oscillation peak. Dashed lines indicate the locking boundary. (d) Same as (a) with $I=2.3 \mathrm{~mA}$. (e) Same as (b) with $I=2.3 \mathrm{~mA}$. (f) Same as (c) with $I=2.3 \mathrm{~mA}$.

For current close to the transition $(I=2.8 \mathrm{~mA})$, synchronization again results in a reduction in linewidth, and corresponding increase in oscillation amplitude (Fig. 37 (b)). Here, the reduction of FWHM (approximately four orders of magnitude) is not as dramatic as when the oscillator is driven below the red-shifting transition, which resulted in a five order of magnitude reduction. However, the enhancement of the oscillation characteristics occured simultaneously with the transition to synchronization.

\subsubsection{Theoretical Model of Hysteretic Synchronization}

Our experimental results confirm the prediction of synchronization hysteresis made by Bonin et al. [61]. However, the original theoretical model is unable to explain the observed asymmetry of the hysteresis. The failure of the model to adequately describe reality arises from its assumption that the auto-oscillation frequency is independent of the oscillation amplitude. To understand the origin of asymmetry, we performed numerical simulations of synchronization taking into account the strong nonlinear dependence of the auto-oscillation frequency on the bias current. Our simulations 
were based on the nonlinear auto-oscillator model [60] for the complex oscillation amplitude $c(t)$ :

$$
\frac{d c}{d t}+i \omega_{0}(p) c+\left[\Gamma_{0}-\sigma I_{0}(1-p)\right] c=\gamma h_{e} e^{-i \omega_{e} t}
$$

Here, $\gamma$ is the gyromagnetic ratio, $p=|c|^{2}$ is the dimensionless precession power, $\omega_{0}(p)$ is the autooscillation frequency, $\Gamma_{0}$ is the natural positive magnetic damping which, for simplicity, is assumed independent of $p$. The parameter $\sigma$ was defined by equation (4b) in Ref. [60], such that $\sigma I_{0}(1-p)$ is the negative damping induced by the spin transfer. The right-hand side of equation (3.4) represents the action of the driving microwave magnetic field of amplitude $h_{e}$ and frequency $\omega_{e}$. We also make the simplifying assumption that we can neglect the ellipticity of the magnetization precession. This assumption is equivalent to an effective renormalization of the driving amplitude $h_{e}$, and does not qualitatively modify the results. To model the experimental geometry that displays the largest hysteresis, the driving field is oriented orthogonal to the magnetization precession axis.

The coefficient $\sigma$ in the relationship between the current and the spin torque is determined predominantly by the spin-polarization efficiency of the polarizing layer. We chose $\sigma=2.0 \mathrm{~ns}^{-1} \mathrm{~mA}^{-1}$, which corresponds to the dimensionless spin-polarization efficiency $\varepsilon \simeq 0.4$. The damping rate $\Gamma_{0}$ is determined not only by Gilbert damping ( $\alpha \simeq 0.01$ ) [58] but also incorporates the effects of radiative damping due to spin wave propagation away from the area of the nano-contact. In our simulations, we chose $\Gamma_{0}=4.0 \mathrm{~ns}^{-1}$ to reproduce the experimental value of the auto-oscillation onset current $I_{t}=\Gamma_{0} / \sigma=2.0 \mathrm{~mA}$. The dependence of the power of the stationary auto-oscillation on the bias current was calculated from equation (3.4) as $p_{0}=\left(I_{0}-I_{t}\right) / I_{0}$.

To verify the validity of our model for the analysis of synchronization hystersis, we initially assumed that the oscillation frequency was linear in bias current, similar to the model of Bonin et. al. With this approximation, the simulations were able to produce symmetric hysteresis, in agreement with the results of Ref. [61]. To model the effect of the actual nonlinear properties of our devices, we analyzed the experimental relationship (Fig. 33 (c)) between the auto-oscillation frequency and the bias current, yielding the dependence $\omega_{0}(p)$ of the frequency on the oscillator power, without any additional assumptions. We fitted this dependence by

$$
\omega_{0}(p)=54.5+(1.16-7.85 p)[1+\tanh (38.5 p-11.3)]
$$




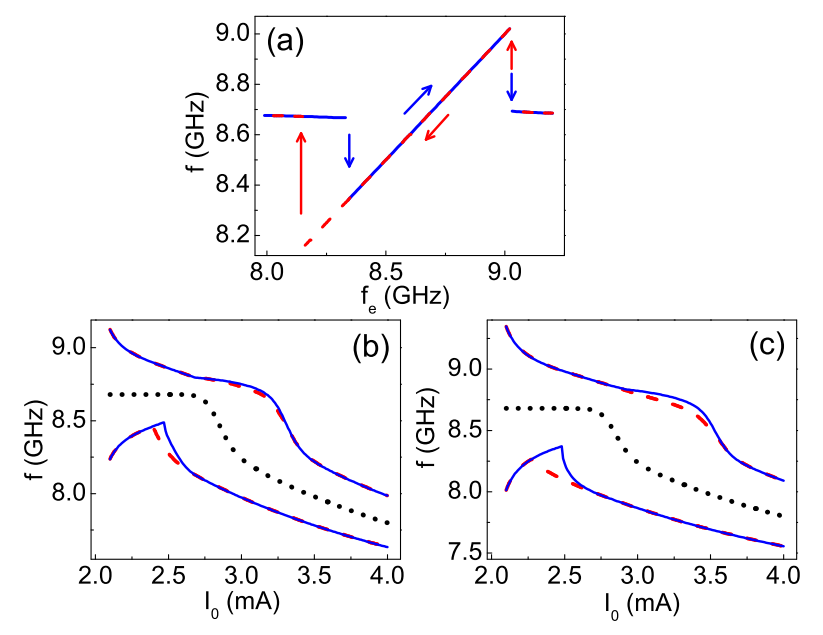

Figure 39: (a) Numerical simulations of the STNO synchronization with increasing (blue solid curve) and decreasing (red dashed curve) driving frequency $f_{e}$, at $I_{0}=2.4 \mathrm{~mA}, h_{e}=30$ Oe. (b) Calculated dependence of the synchronization limits on $I_{0}$ for $h_{e}=20$ Oe. Blue solid (red dashed) curves show the synchronization limits for increasing (decreasing) $\omega_{e}$. (c) Same as (b) for $h_{e}=30$ Oe. Black dotted curves shows the free-running frequency.

where $\omega_{0}$ is expressed in $\mathrm{ns}^{-1}$, which provides an excellent approximation for the experimental data (see Fig. 35).

One example of the simulated synchronization at $I_{0}=2.4 \mathrm{~mA}, h_{e}=30 \mathrm{Oe}$, for two opposite directions of the driving frequency sweep, can be observed in Fig. 39 (a). In accordance with the experimental data, the simulation exhibits noticable hysteresis only at the lower synchronization limit. Additionally, the unlocked oscillation frequency is approximately constant up to the synchronization transition, reproducing the absence of frequency pulling in the experimental data. Both of these features are in remarkable agreement with the experimental results(Fig. 34).

The theoretical model is able to reproduce in the most important and prominent features of the experimental data, as demonstrated in Figs. 39 (b), and 39 (c) for two values of the driving field. In our simulations, the synchronization hysteresis appeared at driving field amplitude exceeding a threshold $h_{t} \approx 15$ Oe. Simulation at $h_{e}=20$ Oe just above $h_{t}$ (Fig. 39 (b)) yielded the average width of the synchronization interval $\Delta f_{e} \approx 500 \mathrm{MHz}$, which was close to the damping rate of the oscillations $\Gamma_{0} /(2 \pi) \approx 600 \mathrm{MHz}$. Additional simulations performed for different values of $\Gamma_{0}$ showed that the hysteresis generally appears at driving field amplitudes exceeding the threshold 
value determined by $\Delta f_{e} \approx \Gamma_{0} /(2 \pi)$. The oscillator's nonlinearity contributes to the emergence of hysteresis through the nonlinear enhancement of the synchronization range [56, 60].

Increasing the driving microwave field $h_{e}$ above the threshold (Fig. 39 (c) for $h_{e}=30$ Oe), we observe that the range of the bias current where hysteresis appears is broadened for both synchronization boundaries, producing a striking similarity with the experimental data in Fig. 35 (d). The simulated synchronization exhibits a large hysteresis at the lower synchronization boundary, at currents below the point of the maximum slope $\left|d \omega_{0}(p) / d p\right|$. A small hysteresis also appears at the upper synchronization boundary over a broad range of the bias current, above the point of the maximum slope $\left|d \omega_{0}(p) / d p\right|$. These features are in excellent agreement with the experimentally observed behaviors, confirming that the observed asymmetry of the hysteresis is caused by the strong nonlinearity of the dependence of the auto-oscillation frequency on the oscillation power.

\subsubsection{Conclusions}

In summary, we have demonstrated asymmetric hysteretic synchronization of a nonlinear magnetic nano-oscillator by a microwave magnetic field. Our observations indicate that the hysteresis is correlated with the region of large nonlinear red frequency shift. Simulations based on the model of a strongly nonlinear auto-oscillator, incorporating the observed dependence of frequency on the bias current, yielded good semi-quantitative agreement with the most important features of the experiment. 


\section{Chapter 4}

\section{Magnetic Ordering in Nanoscale Systems}

\subsection{Effect of Antiferromagnetic Ordering on Current-Induced Switching}

\subsubsection{Introduction}

Current-induced magnetic switching (CIMS) in magnetic nanopillars caused by spin transfer (ST) from the polarized current $I$ to the magnetic moments is an important mechanism for the operation of nanomagnetic devices. [15] The ability to fabricate nanopillars with reproducible, well-controlled geometry and magnetic properties is critical for the understanding of the current-induced behaviors, and for efficient device applications.

Oxidation of the usual Permalloy $=\mathrm{Py}=\mathrm{Ni}_{80} \mathrm{Fe}_{20}$ nanopillars can result in reduced thermal stability at room temperature $295 \mathrm{~K}(\mathrm{RT})$, and increased magnetic damping at low temperatures. $[63,6]$ Both effects are undesirable for magnetic memory applications, which require a combination of high stability and small damping to facilitate CIMS. To eliminate oxidation, protective coating of nanopillars with $\mathrm{AlO}_{x}$ was developed. [6] This procedure requires highly precise oxidation of $\mathrm{Al}$ to avoid partial shorting of nanopillars by unoxidized $\mathrm{Al}$, or over-oxidation leading to formation of magnetic 

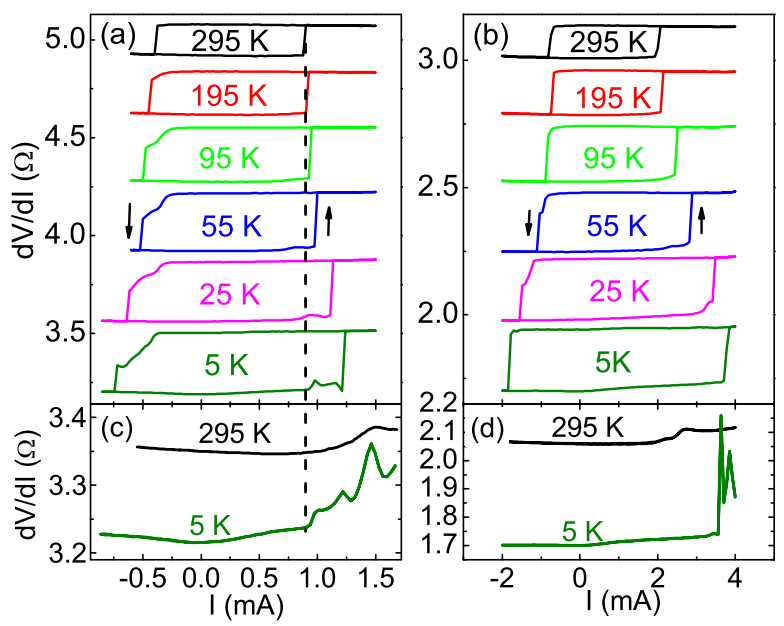

Figure 40: (a,b) $d V / d I$ for samples $A, B$ at $H=20$ Oe and the labeled values of $T$. Curves are offset for clarity. Arrows show scan directions. (c,d) same as (a,b), at $H=500$ Oe.

oxides. Because of these difficulties, it is desirable to develop alternative protection techniques.

Here, we describe a fabrication procedure involving protection of nanopillars with silicon, also serving as an insulating layer between sample leads. Our procedure is simpler than the one involving $\mathrm{AlO}_{x}$, and completely eliminates both the exposure of the nanopillar to oxygen and the possible shunting through the coating. It is thus suitable not only for metallic nanopillars, but also for higherresistance tunnel junctions. We present measurements of CIMS demonstrating the effectiveness of our protection procedure, relate our results to the published measurements, and discuss their implications for the mechanism of CIMS.

\subsubsection{Experimental Results and Discussion}

For details on the fabrication procedure, please see the section on nanopillars for current induced switching. In sample $A$, the current $I_{S}^{+}(T)$ for switching from the low-resistance parallel $(\mathrm{P})$ to the high-resistance antiparallel (AP) state increased from $0.90 \mathrm{~mA}$ at RT to $1.23 \mathrm{~mA}$ at $5 \mathrm{~K}$, with the most significant variation at $T<50 \mathrm{~K}$ (Fig. 40(a)). The current $I_{S}^{-}$for switching from AP to P state decreased from $-0.38 \mathrm{~mA}$ at RT to $-0.73 \mathrm{~mA}$ at $5 \mathrm{~K}$. These variations can originate from the reduced thermal activation, changes of spin-dependent transport properties, [64] or increased damping due to oxidation. $[63,6]$ 

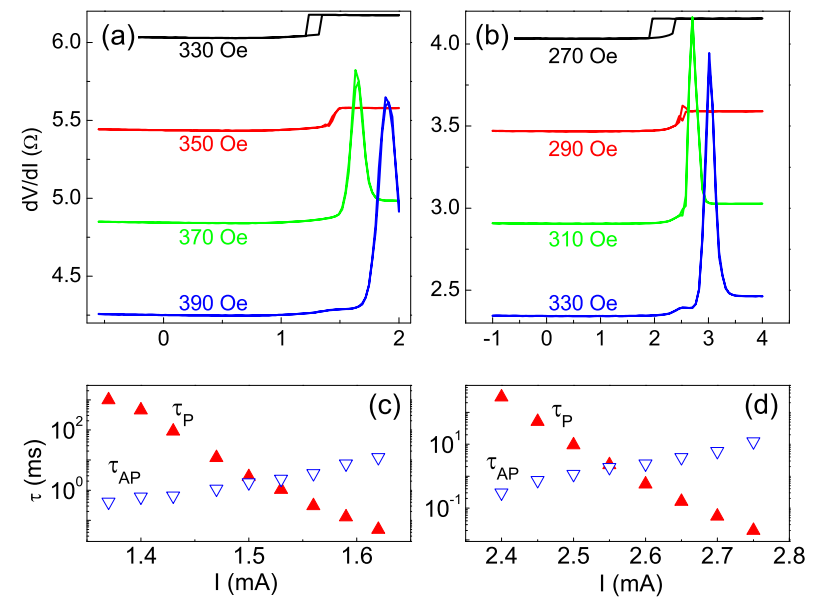

Figure 41: (a,b) $d V / d I$ for samples $A, B$ at the labeled values of $H$. Curves are offset for clarity. (c) Dependence of average dwell times on $I$ in P state (solid symbols) and AP state (open symbols) for sample $A$, at $H=360$ Oe. (d) same as (c), for sample $B$ at $H=285$ Oe.
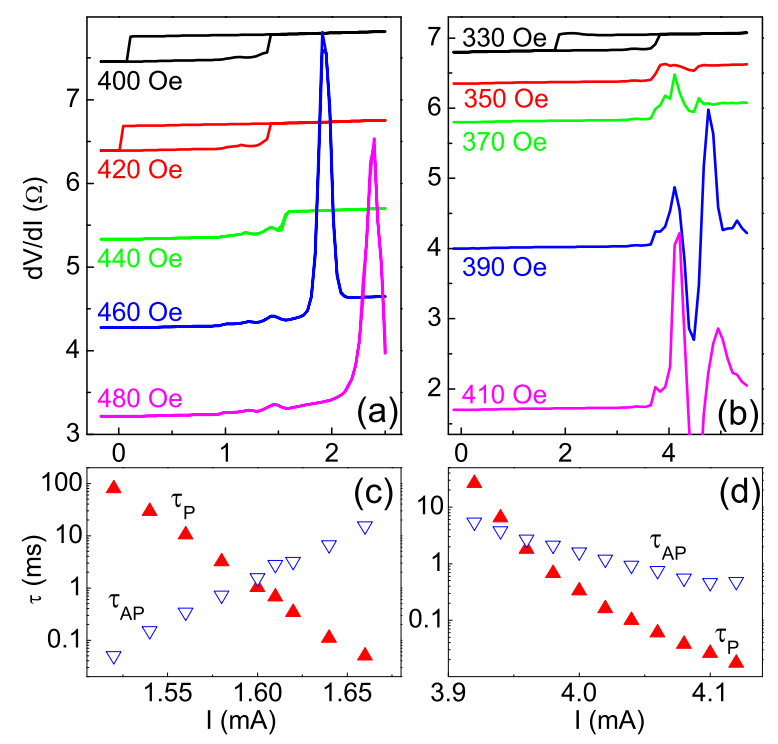

Figure 42: (a,b) $d V / d I$ for samples $A, B$ at the labeled values of $H$. Curves are offset for clarity. (c) Dependence of average dwell times on $I$ in P state (solid symbols) and AP state (open symbols) for sample $A$, at $H=448$ Oe. (d) same as (c), for sample $B$ at $H=370$ Oe. 
At $H=500$ Oe exceeding the coercive field of the nanopillar $H_{C}=430$ Oe at $5 \mathrm{~K}$, the $d V / d I$ sharply increased at the onset of current-induced precession $I_{C}^{+}=0.92 \mathrm{~mA}$ (Fig. 40(c)). The 20 Oe data show a similar increase, indicating large-amplitude precession before reversal at small $H$. The dotted vertical line shows that the onset of precession does not significantly depend on $T$, and coincides with $I_{S}^{+}(R T)$. [65] The same relationship between $I_{S}^{-}$and the precession onset current in the AP state $I_{C}^{-}$is also apparent from the $d V / d I$ data in Fig. 40(a). Therefore, the increase of $I_{S}$ at low $T$ can be attributed entirely to thermal effects, with no evidence for increased damping due to nanopillar oxidation.

We also compare CIMS in sample $A$ with a nearly identical nanopillar protected by $\mathrm{AlO}_{x}$ coating. From Fig. 2(a) of reference [6], $I_{S}^{-}=-2.0 \mathrm{~mA}, I_{C}^{-}=-0.7 \mathrm{~mA}, I_{S}^{+}=2.3 \mathrm{~mA}, I_{C}^{+}=1.25 \mathrm{~mA}$ at $4.2 \mathrm{~K}$. The corresponding $5 \mathrm{~K}$ values for sample $A$ are $-0.73 \mathrm{~mA},-0.38 \mathrm{~mA}, 1.23 \mathrm{~mA}$, and $0.92 \mathrm{~mA}$. To eliminate the effects of different sample areas, these characteristic currents can be multiplied by the $\mathrm{MR} \Delta R=0.23 \Omega$ for $\mathrm{AlO}_{x}$-coated sample, and $\Delta R=0.32 \Omega$ for sample $A$. [66] The values $(|I| \Delta R)$ for sample $A$ are $0.23 \mathrm{mV}, 0.12 \mathrm{mV}, 0.39 \mathrm{mV}$, and $0.29 \mathrm{mV}$, with $I=I_{S}^{-}, I_{C}^{-}, I_{S}^{+}$, and $I_{C}^{+}$, respectively. They are similar to or smaller than the corresponding values $0.46 \mathrm{mV}, 0.16 \mathrm{mV}$, $0.53 \mathrm{mV}$, and $0.29 \mathrm{mV}$ for the $\mathrm{AlO}_{x}$-coated sample, indicating negligible effects of oxidation in sample $A$.

For sample $B, I_{S}^{-}$decreased from $-1.05 \mathrm{~mA}$ at $\mathrm{RT}$ to $-1.81 \mathrm{~mA}$ at $5 \mathrm{~K}$, and $I_{S}^{+}$decreased from $-1.05 \mathrm{~mA}$ at RT to $-1.81 \mathrm{~mA}$ at $5 \mathrm{~K}$ (Fig. 40 (c)). The RT values are two times larger than for sample $A$, despite similar values of $\Delta R(0.16 \Omega$ for $A$ vs $0.13 \Omega$ for $B)$. In contrast to sample $A$, $I_{C}^{+}$increased at low $T$, closely following $I_{S}^{+}$(Fig. 40(d)). A similar relation between $I_{S}^{-}$and $I_{C}^{-}$is apparent from the $d V / d I$ curves in Fig. 40(c), not exhibiting precession before switching, or showing an onset $I_{C}^{-}$very close to $I_{S}^{-}$. The $5 \mathrm{~K}$ values $\Delta R I_{C}^{-}=0.43 \mathrm{mV}$ and $\Delta R I_{C}^{+}=0.9 \mathrm{mV}$ for sample $B$ are more than three times larger than for sample $A$, consistent with enhanced low-temperature damping due to oxidation.

To gain further insight into the effects of oxidation, we analyzed reversible CIMS at large $H$ (Figs. 41, 42). At RT, reversible switching was characterized by large peaks at $H>350$ Oe for sample $A$, and at $H>290$ Oe for sample $B$, which are caused by the thermally-activated random transitions between the $\mathrm{P}$ and AP states. Two determine the statistics of these transitions, we 


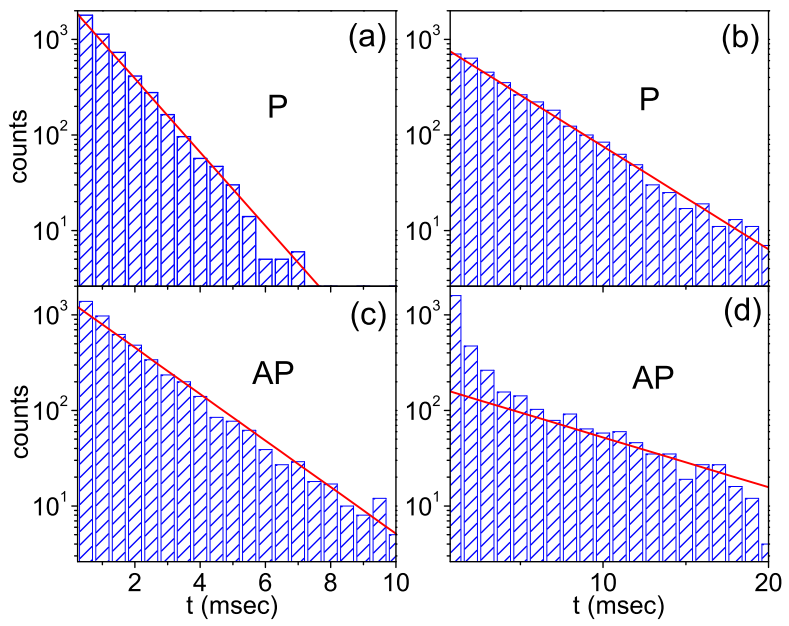

Figure 43: (a,c) Distribution of dwell times in the $\mathrm{P}$ state (a) and AP state (c) for sample $A$ at $H=448 \mathrm{Oe}, I=1.60 \mathrm{~mA}$. (b,d) same as (a,c), for sample $B$ at $H=370 \mathrm{Oe}, I=3.96 \mathrm{~mA}$. Solid lines: best linear fits on log-linear scale.

performed time-resolved measurements of the voltage across the device with a digitizing osilloscope. For each measurement, the acquisition time was adjusted so that a statistically significant number of switching events were contained in a 256,000-point trace. The average dwell time $\tau_{P}$ in the $\mathrm{P}$ state decreased, and the average dwell time $\tau_{A P}$ in the AP state increased with increasing $I$ (Figs. 41(c,d)), resulting in a peak at $\tau_{A P}=\tau_{P} .[65]$

At $5 \mathrm{~K}$, sample $A$ exhibited a similar reversible switching peak due to the decreasing $\tau_{P}$ and increasing $\tau_{A P}$ (Figs. 42(a,c)). In contrast, sample $B$ showed irregular variations of $d V / d I$, but no reversible switching peak (Fig. 42(b)). Anomalous variations of $d V / d I$ at large $H$ were also seen in naturally oxidized nanopillars. [6] We clarified these behaviors by measurements of reversal statistics, showing that both $\tau_{P}$ and $\tau_{A P}$ decreased with increasing $I$ (Fig. 42(d)).

The FokkerPlanck equation describes the time evolution of the probability density function of an observable [67]. Switching statistics can be described by incorporating spin torque into the Fokker-Planck equation, yielding [68]

$$
\tau(I)=\tau_{0} \exp \left(E_{b}\left[1-I / I_{C}\right] / k_{B} T\right),
$$

where $\tau_{0} \approx 10^{-9} \mathrm{~s}$ is the inverse attempt rate, $E_{b}$ is the activation barrier, and $k_{B}$ is the Boltzmann 
constant. Formula 4.1 is valid only for $I<I_{C}$, and is not strictly applicable for activation from the P state at $I>I_{C}^{+}$in Figs. 41(c,d). Nevertheless, it qualitatively describes the observed exponential reduction of $\tau_{P}$ with $I$. For activation from the AP state, Eq. 4.1 with $I_{C}=I_{C}^{-}<0$ predicts an exponential increase of $\tau_{A P}$ with $I$, consistently with RT data for both samples, and $5 \mathrm{~K}$ data for sample $A$. The failure of this formula to describe $\tau_{A P}(I)$ in sample $B$ at $5 \mathrm{~K}$ suggests that reversal occurs over multiple activation barriers. Antiferromagnetic (AF) $\mathrm{NiO}$ formed by the oxidation of the nanopillar has a weak magnetic anisotropy, which is generally insufficient to stabilize its magnetic structure in contact with a ferromagnet. $[69,70]$ Local pinning by the fluctuating AF moments likely produces multiple activation barriers. At large $H$, the activation barrier in the AP state is significantly smaller than in the P state, resulting in larger relative fluctuations of the barrier caused by the same AF pinning, and leading to anomalous behaviors of $\tau_{A P}$.

Both samples at RT, and sample $A$ at $5 \mathrm{~K}$ exhibited an exponential distribution of dwell times in $\mathrm{P}$ and AP states, as expected for random thermally activated reversal over a single activation barrier (Figs. 43(a,c) for sample $A$ at $5 \mathrm{~K}$ ). [68] The dwell times of sample $B$ in the $\mathrm{P}$ state at $5 \mathrm{~K}$ are also well described by the exponential distribution (Fig. 43(b)). However, the dwell times of sample $B$ in the AP state do not follow a single exponential distribution, due to the anomalously large number of low-dwell counts (Fig. 43(d)). This behavior can be attributed to the multiple-barrier activation with at least two significantly different characteristic dwell times, consistent with the fluctuating pinning by the AF oxide discussed above. We note that a similar absence of the reversible switching peak, anomalous dependence of dwell times on $I$, and non-exponential reversal statistics were also observed in nanopillars exchange-biased by a thin AF FeMn, supporting the origin of anomalies in sample $B$ from the AF surface oxide. [71]

\subsubsection{Conclusions}

In summary, we demonstrated that magnetic nanopillars can be efficiently protected from oxidation by sputtered insulating silicon, resulting in excitation onset current nearly independent of temperature. In contrast, oxidized nanopillars exhibit larger switching currents, increased excitation onset currents at low temperatures, and anomalous low-temperature statistics of current-induced reversal. These behaviors provide key signatures for the effects of oxidation, which can be conveniently used 
in future studies to characterize the quality of magnetic nanodevices. We expect our passivation technique to be transferrable to other nanomagnetic devices such as tunnel junctions.

\subsection{Modification of Exchange Bias in CoO by Pt Doping}

\subsubsection{Introduction}

The asymmetry of hysteresis in ferromagnet/antiferromagnet (F/AF) bilayers, known as the exchange bias (EB) effect, [4] has been extensively studied and utilized in magnetic devices. The EB originates from the stable uncompensated AF magnetic moments at the $\mathrm{F} / \mathrm{AF}$ interface, which exert an exchange force on $\mathrm{F}$ approximately equivalent to an effective field $H_{E}$ [72]. The interest in the EB is stimulated by the diversity of magnetic behaviors due to a competition of several generally comparable energies: anisotropies of $\mathrm{F}$ and $\mathrm{AF}$, volume and interface exchange energies, and the thermal energy. Understanding how the interplay of these energies affects the EB can lead to increased tunability of the magnitude, stability, and temperature dependence of the effect for device applications.

The surface of AF films in a single-domain state is generally compensated, [73] and thus cannot produce EB [74]. The EB is therefore associated with a multidomain state, in which the magnetic domains are separated either by domain walls, [75] or atomically sharp boundaries. The AF can then be approximately described by a collection of single-domain grains weakly linked across their boundaries [76]. Regardless of the microscopic magnetic structure of domain boundaries, their energy is generally expected to be lowered by pinning on defects and crystalline grain boundaries. In the following, we will collectively refer to the density of defects lowering the multidomain state energy as the granularity of AF.

The existence of uncompensated AF magnetic moments is facilitated by the multidomain state of AF, and therefore their density (and thus EB) should generally increase when the granularity is increased. An inverse relationship between crystalline sizes and EB has indeed been demonstrated for polycrystalline films [77]. However, sufficiently small AF grains can become magnetically unstable due to their small anisotropy energy, in which case they do not contribute to EB.

Despite the importance of AF granularity, few experiments have directly tested its relationship 
with EB, and a complete picture has not yet emerged. Simple oxide AFs are particularly attractive for such studies, because high-quality single crystals or epitaxial films can be fabricated. The effects of enhanced granularity in $\mathrm{CoO}$ were studied either by introducing Co vacancies, [78] or by dilution with nonmagnetic Mg impurities [78, 79]. The defects were presumed to break the bonds responsible for the magnetic interactions between neighboring $\mathrm{AF}$ atoms. In these studies, $H_{E}$ increased at least for moderate doping, which was explained either by the lowering of the domain wall energies, [78] or by the increased density of uncompensated interfacial AF moments [79]. However, the granularity introduced by the defects was superimposed on the unknown grain structure of polycrystalline $\mathrm{CoO}$ in one study, and a twinned structure in the other, complicating direct analysis. Additionally, these studies revealed surprisingly little evidence for decreased stability of AF grains whose sizes were effectively decreased by doping.

We report a comparative study of EB in epitaxial and polycrystalline $\mathrm{CoO}$ films doped with up to 35 atomic percent of $\mathrm{Pt}$. The electronic structure of $\mathrm{Pt}$ is different from $\mathrm{Co}$, so that its incorporation in the $\mathrm{CoO}$ matrix must efficiently increase granularity. We see effects of both the increased spin density and decreased AF stability. Our measurements show a significant difference between the effects of doping in epitaxial and polycrystalline films. These results are explained by the superposition of contributions from the crystalline and compositional inhomogeneities.

\subsubsection{Sample Fabrication}

Multilayers with structure $\mathrm{Co}_{1-x} \mathrm{Pt}_{x} \mathrm{O}(6) \mathrm{Co}(8) \mathrm{Cu}(5) \mathrm{Py}(5) \mathrm{Pt}(2)$, where $x=0,0.15,0.25$, and 0.35, were deposited by dc magnetron sputtering in a chamber with a base pressure of $5 \times 10^{-9}$ Torr. All thicknesses are in nanometers. The top $\mathrm{Py}=N i_{80} \mathrm{Fe}_{20}$ layer formed a pseudo spin valve with the exchange-biased Co, enabling electronic measurements of magnetic hysteresis via Giant Magnetoresistance (GMR). The AF $\mathrm{Co}_{1-x} \mathrm{Pt}_{x} \mathrm{O}$ was deposited by co-sputtering from Co and $\mathrm{Pt}$ sources in Ar/O mixture at $\mathrm{O}_{2}$ partial pressure of 0.1 mTorr, and Ar pressure of 4.6 mTorr. The partial oxygen pressure was adjusted until films of $\mathrm{Co}$ and $\mathrm{Co} / \mathrm{Pt}$ sputtered in $\mathrm{Ar} / \mathrm{O}$ mixture exhibited negligible ferromagnetic response measured by vibrating sample magnetometry. This technique minimized overoxidation, which can lower the Neel temperature [80]. All deposition rates were monitored by a quartz crystal oscillator. The deposition rate of $\mathrm{CoO}$ was kept at about $0.1 \mathrm{~nm} / \mathrm{s}$, while the 

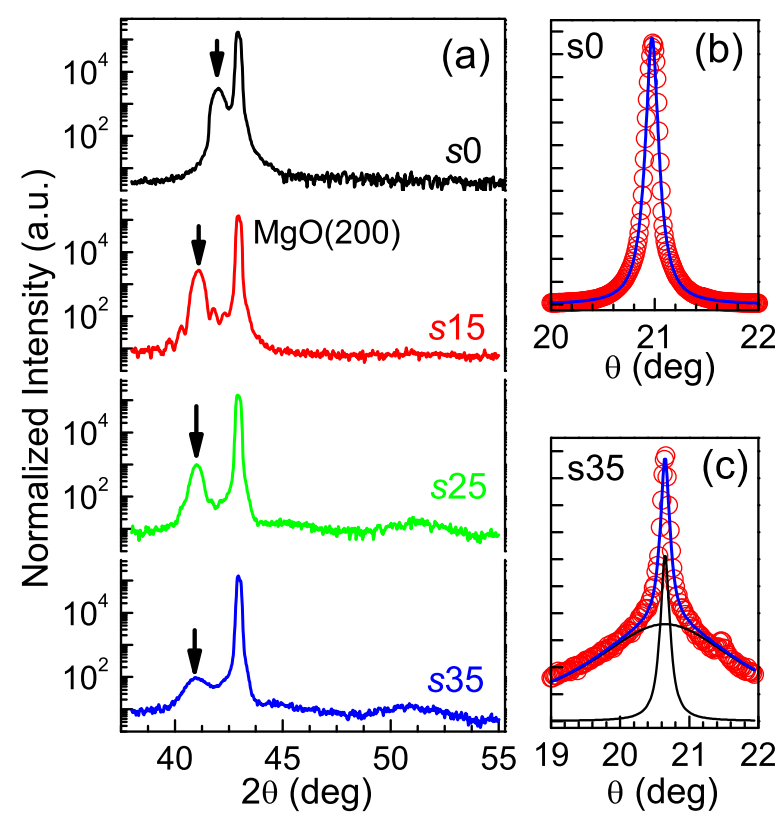

Figure 44: (a) $\theta-2 \theta$ x-ray scans of epitaxial samples as labeled. Arrows show $\operatorname{CoOPt}(200)$ peaks. (b) $\mathrm{CoO}(200)$ rocking curve for $s 0$ (symbols), fitted by a Lorenzian with FWHM of $0.15^{\circ}$ (solid curve). (c) Same for $s 35$, fitted by a sum of two Lorenzians with FWHM of $0.08^{\circ}$ and $1.35^{\circ}$, as shown by three curves.

deposition rate of $\mathrm{Pt}$ was adjusted to the desired level.

Polycrystalline samples were deposited on thermally oxidized Si substrates at room temperature $295 \mathrm{~K}$ (RT). Epitaxial CoOPt films were deposited on polished epi-ready $\mathrm{MgO}(100)$ substrates at $150^{\circ} \mathrm{C}$ after brief surface cleaning by Ar ion bombardment. Subsequent deposition of the remaining multilayer was performed in 4.3 mTorr of purified Ar, after pumping out oxygen and cooling the sample to below $80^{\circ} \mathrm{C}$. The polycrystalline and epitaxial samples are labeled $p x$ and $s x$, respectively, where $x$ is the Pt content. The magnetic hysteresis was measured via GMR by four-probes in Van der Pauw geometry between $5 \mathrm{~K}$ and $295 \mathrm{~K}$ (RT), after initial cooling in field $H=500$ Oe from RT to $5 \mathrm{~K}$. 


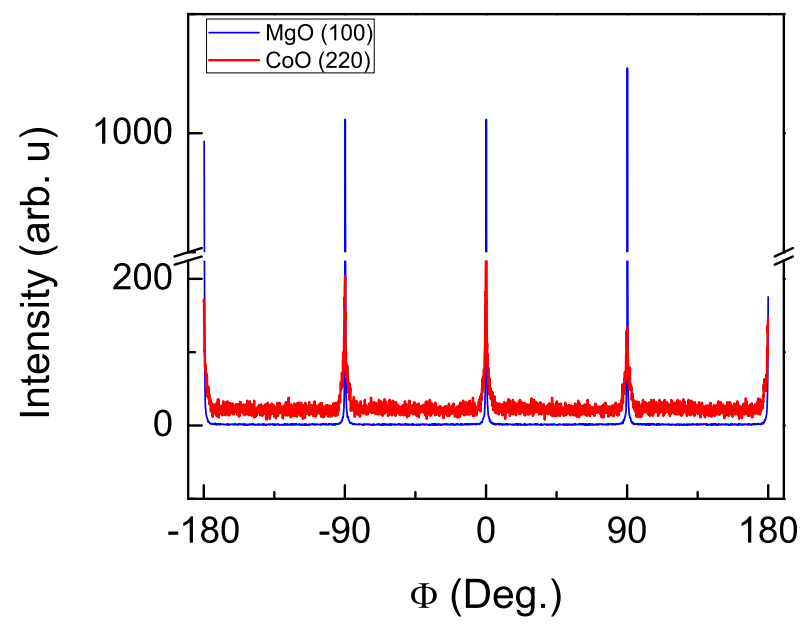

Figure 45: Polar x-ray scan of $\mathrm{CoO}$ (220) on $\mathrm{MgO}$ (100). Red curve is the film, blue curve is the substrate.

\subsubsection{Experimental Results and Discussion}

Before focusing on the magnetic properties of our samples, we first discuss the effect of $\mathrm{Pt}$ on crystalline properties of $\mathrm{AF}$, critical to understanding the granularity of our samples. We have performed extensive characterization by x-ray diffraction using Cu K- $\alpha$ line. Fig. 44(a) shows Bragg diffraction for the four epitaxial samples. The $\mathrm{CoO}(200)$ peak is at $2 \theta=42.01^{\circ}$, to the left of the $\mathrm{MgO}(200)$ peak at $2 \theta=42.93^{\circ}$. The in-plane coherence of AF films was verified by polar scans of the $\mathrm{CoO}(220)$ peaks (Fig. 45). The FWHM of the rocking curve for $\mathrm{CoO}(200)$ was $\Delta \theta=0.15^{\circ}$ (Fig. 44(b)). As the Pt doping level was increased, the $\mathrm{CoO}(200)$ Bragg peak first shifted to lower $2 \theta$ in sample $s 15$, and then decreased in amplitude without further shifts in samples $s 25$ and $s 35$ (Fig. 44(a)).

The correlation lengths in the direction of the scattering vector could be estimated with a Sherrer equation $\xi=0.9 * \lambda /(\cos \theta \Delta 2 \theta(\mathrm{rad}))$, [35] where $\lambda$ is the wavelength of the $\mathrm{Cu} \mathrm{K}-\alpha$ line, and $\Delta 2 \theta$ is FWHM of Bragg peak. The FWHM of the Bragg peaks were 0.5, 0.4, 0.4, and 1.1, for samples for $s 0, s 15, s 25$, and $s 35$, respectively, yielding $\xi=21 \mathrm{~nm}, 27 \mathrm{~nm}, 27 \mathrm{~nm}$, and $10 \mathrm{~nm}$. The first three values indicate long-range order throughout the film thickness. The values of $\xi$ for $s 15$ and $s 25$ are larger than the film thickness, likely due to the effect of finite-size fringes, which are especially pronounced for the sample $s 15$. Their presence indicates that modest Pt doping facilitates nearly 

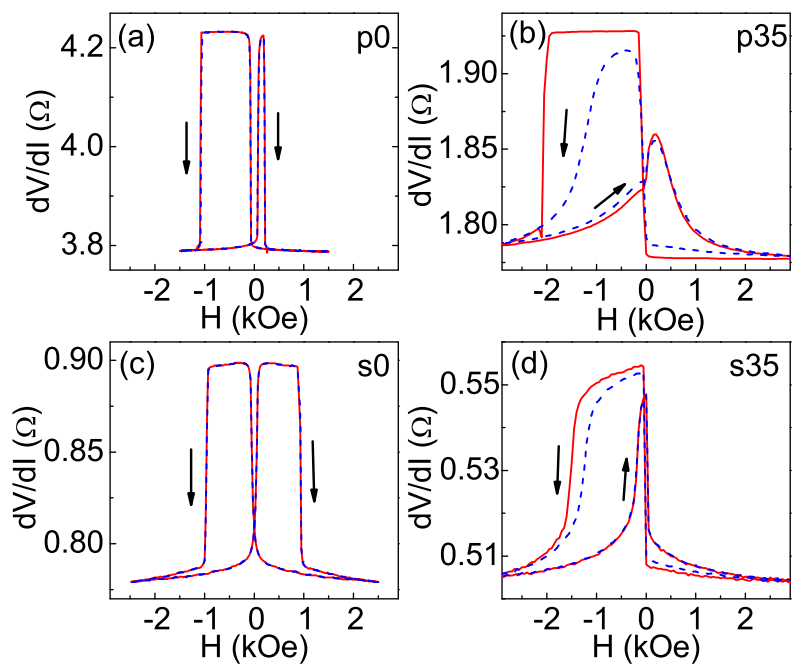

Figure 46: Hysteresis loops acquired immediately after cooling from RT to $5 \mathrm{~K}$ in field 500 Oe: (a) sample $p 0$, (b) $p 35$, (c) $s 0$, (d) $s 35$. Solid curves are for the first loop, and dashed curves are for the second loop. Arrows show scan directions at the reversal points of $\mathrm{Co}(8)$. The field is in the $\mathrm{Co}[010]$ direction for epitaxial samples.

atomic smoothness of the films. Bragg intensities for samples $s 25$ and $s 35$ exhibit two broad peaks around $2 \theta=45.3^{\circ}$ and $2 \theta=51.2^{\circ}$, which are attributed to strained $\operatorname{Pt}(200)$ and $\operatorname{Pt}(111)$ reflections, respectively. These broad peaks are likely caused by the formation of Pt clusters in films with the highest levels of doping. The rocking curves for Pt-doped samples show a superposition of a sharp peak whose amplitude decreases with doping, and a broad diffuse background increasing with doping, as illustrated in Fig. 44(c) for $s 35$.

The x-ray diffraction results indicate that small concentrations of $\mathrm{Pt}$ in $s 15$ dissolve in $\mathrm{CoO}$ matrix. Pt clusters form at larger Pt concentrations in samples $s 25$ and $s 35$. Their crystalline orientation is in registry with the $\mathrm{CoO}$ matrix, resulting in broad diffraction peaks in specular reflection, and diffuse background in the $\mathrm{CoO}(200)$ rocking curve (Fig. 44(c)) due to a significant local distortion of $\mathrm{CoO}$ matrix around the Pt clusters. This observation will help us identify the origins of some of the magnetic behaviors described below.

Magnetic hysteresis loops were measured via GMR, after in-field cooling to $5 \mathrm{~K}$. The variations of $d V / d I$ corresponded to reversals of $\mathrm{Co}(8)$ and $\mathrm{Py}(5)$. The latter always reversed in a single sharp step at small $H= \pm(5-10)$ Oe, and thus could be easily separated from the reversal of $\mathrm{Co}(8)$ 
typically at significantly larger $H$. The field values at which resistance is halfway between the lowest and the largest value are labeled reversal fields $H^{+}$and $H^{-}$for increasing- $H$ and decreasing- $H$ scans, respectively. The exchange bias field $H_{E}$ and the coercivity $H_{C}$ are defined by $H_{E, C}=\left(H^{+} \pm H^{-}\right) / 2$, and characterize the asymmetry and the overall width of the hysteresis loop.

The consecutive hysteresis loops for the undoped $\mathrm{CoO}$ samples $s 0$ and $p 0$ were nearly identical, as shown in Figs. 46(a),(b), where the two initial loops are indistinguishable. In contrast, Pt doping resulted in increasingly significant training effect - a decrease of the reversal field during consecutive scans [81]. The first two loops for samples $p 35$ and $s 35$ are shown in Figs. 46(b) and (d), respectively. Subsequent hysteresis curves were identical to the second loop for all the samples. Fig. 46(d) shows the data for $H$ parallel to the $\mathrm{Co}[010]$ direction. We also performed similar measurements with $H$ in $\mathrm{Co}[011]$ direction, yielding more square hysteresis loops, but similar values of $H_{C}$ and $H_{E}$.

The training effect originates from the instability of the initial AF configuration, which relaxes during the reversals of $\mathrm{F}$ due to the exchange interaction at $\mathrm{F} / \mathrm{AF}$ interface [81]. Its presence in the Pt-doped samples is consistent with the reduced magnetic stability of smaller magnetic grains. We identify grains as the parts of the AF magnetically decoupled from the matrix due to the surrounding Pt defects and/or crystalline grain boundaries. We note that the larger training effect in polycrystalline samples indicates smaller AF grain sizes determined by a combination of crystalline grain boundaries and Pt doping. The hysteresis loop for $p 35$ in panel (b) initially shows a larger asymmetry than the undoped sample $p 0$ in panel (a). This behavior is consistent with the increased density of uncompensated $\mathrm{AF}$ moments at $\mathrm{F} / \mathrm{AF}$ interface due to the increased granularity.

Comparison of top and the bottom panels in Fig. 46 reveals a dramatic difference between the behaviors of polycrystalline and epitaxial samples. The undoped sample $s 0$ exhibits a large $H_{C}$, but negligible $H_{E}$, consistent with the previous studies of $\mathrm{EB}$ in $\mathrm{CoO}(100)$ [82]. This result indicates negligible average uncompensated moment density as expected for single-crystalline surface of $\mathrm{AF}$ with low defect density. In contrast, all of the Pt-doped epitaxial samples exhibited a finite $H_{E}$, as illustrated for $s 35$ in Fig. 46(d). The appearance of EB is consistent with the increase of AF granularity, resulting in a finite uncompensated AF moment density.

Additional information about the effect of $\mathrm{Pt}$ doping on the AF granularity is provided by the temperature dependence of the exchange bias and coercivity, as shown in Fig. 47 for all of 

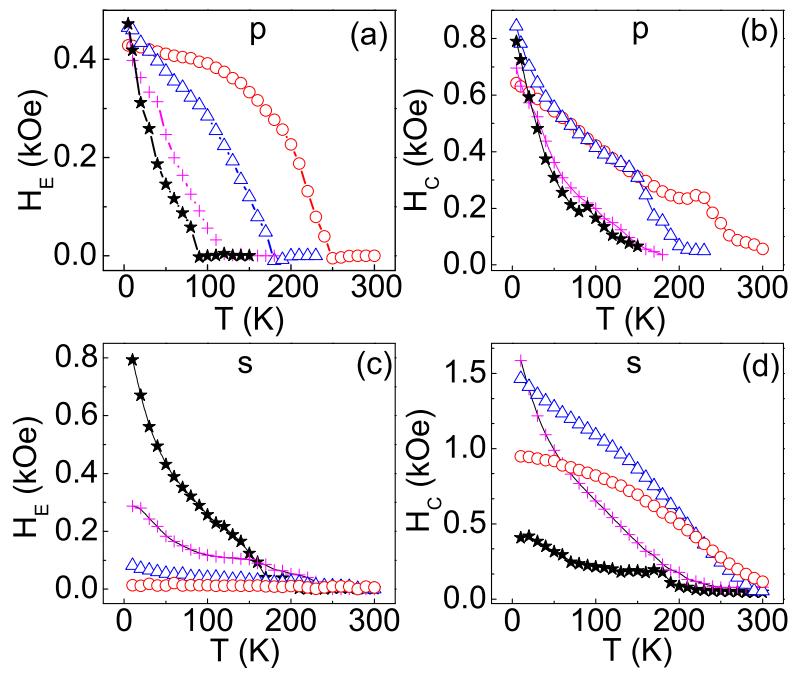

Figure 47: (a,b) Temperature dependence of the exchange bias field $H_{E}$ (a), and coercivity $H_{c}(\mathrm{~b})$, for polycrystalline samples. (c,d) Same as (a,b), for epitaxial samples. Symbol types distinguish samples with different Pt concentration: circles for $x=0$, triangles for $x=15$, crosses for $x=25$, and stars for $x=35$. The field is in the $\mathrm{Co}[010]$ direction for epitaxial samples.

our polycrystalline and epitaxial samples. For the following discussion, we define the blocking temperature $T_{B}$ of $\mathrm{AF}$ by the onset of finite $H_{E}$, which is usually correlated with a sharp increase or a bump in $H_{C}$. This bump is caused by torque due to the unstable AF grains, which at lower $T$ freeze out and instead contribute to $H_{E}$ [81]. The data for polycrystalline samples (Figs. 47(a,b)) exhibit a monotonic decrease of $T_{B}$ with increasing Pt doping, consistent with a decreased stability of smaller AF grains. It is also possible that $\mathrm{Pt}$ doping also decreases the Neel temperature of $\mathrm{CoO}$, which sets the upper limit for $T_{B}$. Lower values of $T_{B}$ lead to smaller $H_{E}$ over the entire measured temperature range except $T=5-30 \mathrm{~K}$, where $H_{E}$ becomes similar for all the samples. However, recalling that the training effect is more significant in doped samples, we conclude that doping increases the uncompensated spin density while decreasing the AF stability.

In contrast to polycrystalline samples, Pt doping of epitaxial samples results in a monotonic increase of $H_{E}$ over nearly the entire measured temperature range (Fig. 47(c)). The opposite dependence of $H_{E}$ on $\mathrm{Pt}$ doping in epitaxial and polycrystalline samples is the main result of this work. The largest values of $H_{E}$, obtained for $s 35$ at $5 \mathrm{~K}$, exceed those for polycrystalline samples by almost a factor of two. This latter result may indicate that the AF anisotropy is enhanced either due to 
the spin-orbit interaction at the Pt sites, or more likely due to the strain induced by incorporation of $\mathrm{Pt}$ atoms and clusters into the epitaxial $\mathrm{CoO}$ matrix. In contrast, strain is efficiently relaxed at the grain boundaries in polycrystalline samples. An additional possibility is that the incorporation of Pt clusters facilitates the formation of AF domains, resulting in the formation of $\mathrm{H}_{E}$. We note that $T_{B}$ is larger in epitaxial samples with the same level of doping as polycrystalline ones, which is particularly pronounced for $p 35\left(T_{B}=90 \mathrm{~K}\right)$ and $s 35\left(T_{B}=170 \mathrm{~K}\right)$. These differences are consistent with the larger AF grain sizes in epitaxial samples.

We also note a significantly more complex nonmonotonic dependence of $H_{C}$ on doping in epitaxial samples. A thorough interpretation of these behaviors will require a better general understanding of the enhanced $H_{C}$ in F/AF bilayers. Several possible mechanisms include torques due to the unstable AF grains, $[83,84]$ uniaxial anisotropy due to the flopping of AF moments in stable grains, $[74,76]$ and local anisotropy due to the spatial fluctuations of the uncompensated AF moments [85, 86]. The first two mechanisms increase $H_{C}$ by changing the average anisotropy of ferromagnet, while the latter less explored mechanism can result in pinning of domain walls on inhomogeneities without affecting the average anisotropy.

The temperature dependence of the data shown in Figs. 47(c,d) eliminates the unstable AF grains as the dominant source of enhanced $H_{C}$; as $T$ is lowered, such grains would become stable and begin to contribute to $H_{E}$, which is inconsistent with the large $H_{C}$ but negligible $H_{E}$ over a large temperature range for samples $s 0$ and $s 15$. One can also argue that significant local fluctuations of uncompensated AF moments are unlikely for the compensated $\mathrm{Co}(100)$ surface. We believe that enhanced $H_{C}$ in epitaxial samples is indicative of flopping of the compensated interfacial AF moments in stable grains, due to exchange interaction with F [74]. As Pt doping is increased, the exchange interaction at the $\mathrm{F} / \mathrm{AF}$ interface is increasingly accommodated by the reorientation of AF grains, likely reducing the effects of flopping.

Comparison of the top and the bottom panels in Fig. 47 also reveals that the dependence of $H_{E}$ and $H_{C}$ on doping in polycrystalline samples becomes weaker with increased doping level, such that the $p 25$ and $p 35$ data are very similar. This dependence may be expected due to the clustering of Pt in highly doped samples (Fig. 44(a)), reducing the effect of doping on granularity. In contrast, the effect of increasing $\mathrm{Pt}$ concentration in epitaxial samples is the strongest at high doping levels 
(Figs. 47(c,d)). This indicates that increased granularity may not be the only factor contributing to the EB in doped epitaxial samples. The enhancement of corecivity that coexists with exchange bias in all of our samples indicates that some of the uncompensated AF spins reverse together with the magnetization of ferromagnet. One of the possible contributions to EB enhancement in PT-doped samples may come from increased local anisotropy that results in "freezing" of these spins. Such potentially complex interplay of several contributions to EB warrants more detailed quantitative simulation of this doped AF system. It would be also interesting to investigate the effect of $\mathrm{AF}$ thickness on the granularity and EB in this system.

\subsubsection{Conclusions}

In summary, we have achieved an increased granularity of epitaxial and polycrystalline $\mathrm{CoO}$ films by doping them with up to $35 \%$ of Pt. The increased granularity resulted in a decrease of the blocking temperature and appearance of the training effect. Hysteresis measurements performed between room temperature and $5 \mathrm{~K}$ showed that doping also increases the density of uncompensated $\mathrm{AF}$ magnetic moments, resulting in an increase of exchange bias in epitaxial samples. In polycrystalline samples, the initial increase of exchange bias was compensated by the training effect. The largest exchange bias in epitaxial samples is twice as large as in polycrystalline ones, which may be caused by an increase of the local strain-induced $\mathrm{CoO}$ anisotropy due to the doping. 


\section{Chapter 5}

\section{Engineering of Topologically Nontrivial Materials}

\subsection{Introduction}

Topologically nontrivial states of condensed matter systems have recently attracted significant interest due to their potential spintronic and quantum computing applications [7]. Topological insulators (TIs) are characterized by surface states that are robust with respect to disorder and exhibit high mobility due to the suppression of carrier backscattering $[17,87,9]$. These states are spin-momentum locked, making TIs attractive for spintronic devices. In addition, interfaces between a TI and a superconductor can produce Majorana fermions [11] central to some schemes for fault-tolerant quantum computation.

Development of devices utilizing TIs will require accurate characterization of the surface electronic transport. However, measurements performed so far have been generally dominated by bulk conduction [88, 89]. Among the experimentally studied TI materials [18, 20, 21, 90, 91, 92], $\mathrm{Bi}_{2} \mathrm{Se}_{3}$ exhibits the largest bulk bandgap of $0.3 \mathrm{eV}$. Consequently, compensated $\mathrm{Bi}_{2} \mathrm{Se}_{3}$ is expected to exhibit the smallest bulk contribution to transport, and has become the material of choice for experimental studies.

The contribution of bulk conduction to transport can be reduced by utilizing thin films. Growth 

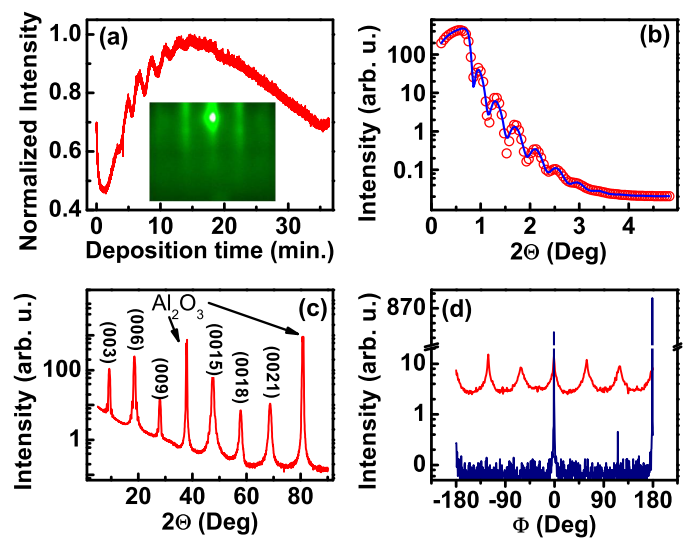

Figure 48: Typical structural characterization results: (a) Intensity oscillations of the specular RHEED peak. Inset: unreconstructed RHEED pattern; (b) Low angle x-ray reflectivity data (red open circles) and fit to data (blue solid curve); (c) $\theta-2 \theta$ x-ray diffraction scan. Substrate peaks are indicated by arrows; (d) Azimuthal x-ray diffraction scan of the $\{105\} \mathrm{Bi}_{2} \mathrm{Se}_{3}$ planes (light red) and of the $\{113\}$ substrate planes (dark blue). All data shown are for a film grown at $T_{S}=140^{\circ} \mathrm{C}$, $\Phi_{r}=11.8$.

of thin $\mathrm{Bi}_{2} \mathrm{Se}_{3}$ films has been demonstrated on several types of substrates [10, 23, 24, 25, 26]. For practical purposes, the substrate must be insulating to minimize its contribution to conduction. Additionally, the interface of a trivial insulating substrate with the TI film is expected to exhibit topological states with properties similar to the free TI surface, while being protected from the effects of oxidation. Characterization of these interfacial states can be facilitated by a transparent substrate that allows optical access to the interface.

Here, we demonstrate that high quality $\mathrm{Bi}_{2} \mathrm{Se}_{3}$ thin films can be grown by molecular beam epitaxy (MBE) on $\mathrm{Al}_{2} \mathrm{O}_{3}$, which is both transparent and electrically insulating. We show that the precise control of the film growth parameters provided by MBE enables optimization of important film characteristics such as morphology, crystallinity, carrier concentration and mobility.

\subsection{Growth of Ultra-thin $\mathrm{Bi}_{2} \mathrm{Se}_{3}$ by Molecular Beam Epitaxy}

The films were grown on a-plane $\mathrm{Al}_{2} \mathrm{O}_{3}$ single crystal substrates degreased and annealed in air. MBE growth was performed in a vacuum chamber with a base pressure of $1.2 \times 10^{-10}$ Torr utilizing effusion of high purity Bi and Se source materials. The two key parameters affecting the quality of 
the film are the substrate temperature $\mathrm{T}_{S}$ and the relative atomic $\mathrm{Se} / \mathrm{Bi}$ flux ratio $\Phi_{r}$. To separate these contributions, one set of samples was grown at a fixed $\Phi_{r}=11.8 \pm 0.6$ with the substrate temperature $T_{S}$ varied between $140{ }^{\circ} \mathrm{C}$ and $240{ }^{\circ} \mathrm{C}$. Another set was grown at a fixed $T_{S}=200{ }^{\circ} \mathrm{C}$ with $\Phi_{r}$ varied between 1.5 and 17 . All films were $17 \mathrm{~nm}$ to $20 \mathrm{~nm}$ thick.

\subsection{Results and Discussion}

The surface properties were monitored in-situ by reflection high energy electron diffraction (RHEED). The specular peak intensity exhibited periodic oscillations characteristic of Frank-van der Merwe growth mode [93] with a period consistent with the growth in quintuple layer (QL) steps (Fig. 48(a)), similar to previous studies of epitaxial growth on other substrate materials [10, 23, 24]. The RHEED diffraction patterns were streaky and unreconstructed, with no visible transmission spots (inset in Fig. 48(a)), indicating good surface crystallinity.

Additional characterization was performed ex-situ by x-ray scattering. Fitting the low-angle x-ray reflectivity to an optical scattering model [94] yielded surface roughness parameters of $1 \mathrm{~nm}$ to $1.5 \mathrm{~nm}$, corresponding to $1-1.5$ QLs (Fig. 48(b)). The films were c-axis oriented, as indicated by the $\{003\}$ peaks in the $\theta-2 \theta$ high angle x-ray diffraction with well-defined Kiessig fringes evident around the (003) and (006) peaks (Fig. 48(c)). Epitaxy was confirmed by the six-fold symmetric $\{105\}$ diffraction pattern in azimuthal $\phi$-scans (Fig. 48(d)). The $\mathrm{Bi}_{2} \mathrm{Se}_{3}[100] \| \mathrm{Al}_{2} \mathrm{O}_{3}$ [001] in-plane relationship was established by comparison with the $\{113\} \mathrm{Al}_{2} \mathrm{O}_{3}$ substrate peaks.

In constrast to the crystalline properties, the surface morphology strongly depended on $\Phi_{r}$ and $T_{S}$ (Fig. 49). For $T_{S}=140{ }^{\circ} \mathrm{C}$, the surface exhibited densely packed islands that lacked a distinct shape, and $1 \mathrm{~nm}$ rms roughness consistent with the low angle reflectivity (Fig. 49(a)). For $T_{S}=200{ }^{\circ} \mathrm{C}$, the islands formed triangular prisms with similar roughness (Fig. 49(b)). The size of the islands grew with increasing $T_{S}$. At small $\Phi_{r}=4.9$ and $T_{S}=200{ }^{\circ} \mathrm{C}$, the surface exhibited randomly oriented elongated structures likely formed by Bi-rich phase (Fig. 49(c)). Prism islands started to form at larger $\Phi_{r}$; their size increased with increasing $\Phi_{r}$ (Fig. 49(d)). The triangular shape of the prisms is consistent with the predominantly $p p \sigma$ bonding of $\mathrm{Bi}_{2} \mathrm{Se}_{3}$. The prisms were oriented with $60^{\circ}$ rotations with respect to each other, consistent with the six-fold symmetry of the x-ray $\phi$-scans.

The stoichiometry of the films was characterized by ex-situ x-ray photoelectron spectroscopy 


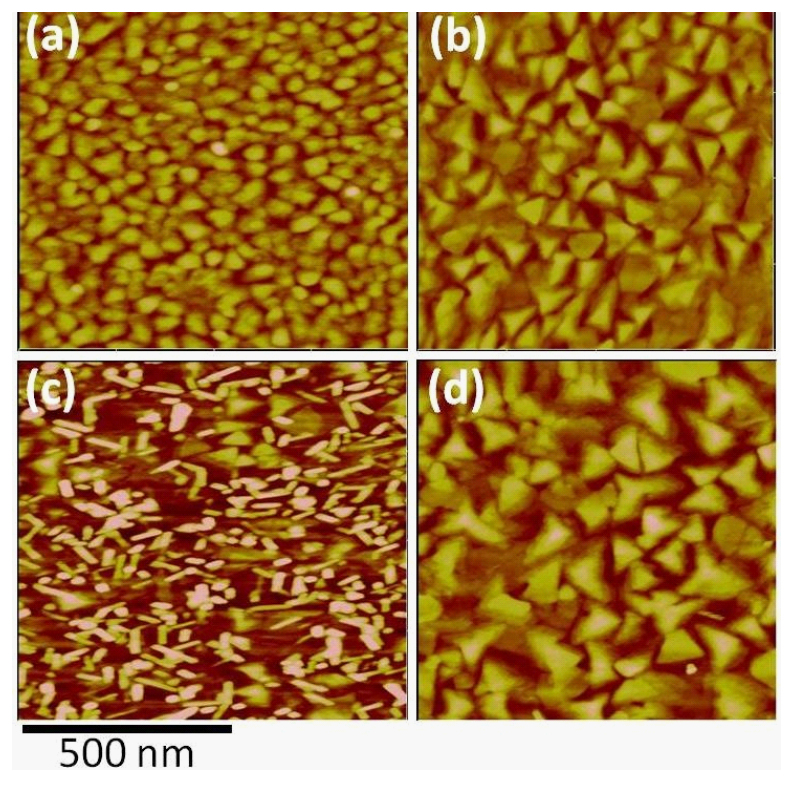

Figure 49: Dependence of the film morphology determined by ex-situ atomic force microscopy on the substrate temperature $T_{S}$ and on the $\mathrm{Se} / \mathrm{Bi}$ flux ratio $\Phi_{r}$ : (a) $T_{S}=140{ }^{\circ} \mathrm{C}, \Phi_{r}=11.5$; (b) $T_{S}=200{ }^{\circ} \mathrm{C}, \Phi_{r}=11.9$; (c) $T_{S}=200{ }^{\circ} \mathrm{C}, \Phi_{r}=4.9$; and (d) $T_{S}=200{ }^{\circ} \mathrm{C}, \Phi_{r}=16.8$. The full z-scale is $10 \mathrm{~nm}$.
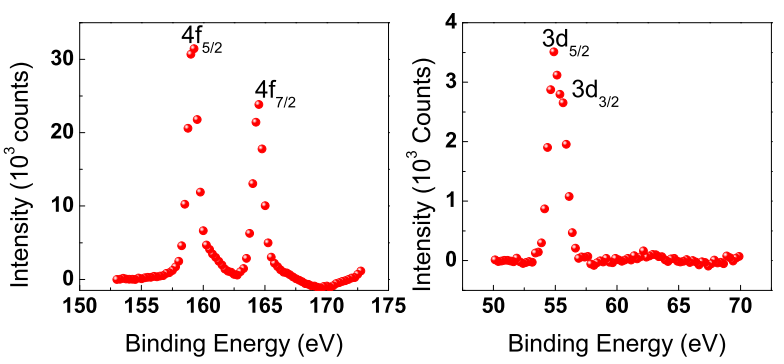

Figure 50: XPS spectra of film grown at $\mathrm{T}_{S}=200^{\circ} \mathrm{C}$ and $\Phi_{r}=12$ (a) Bismuth doublet peaks (b) Selenium doublet peaks. 

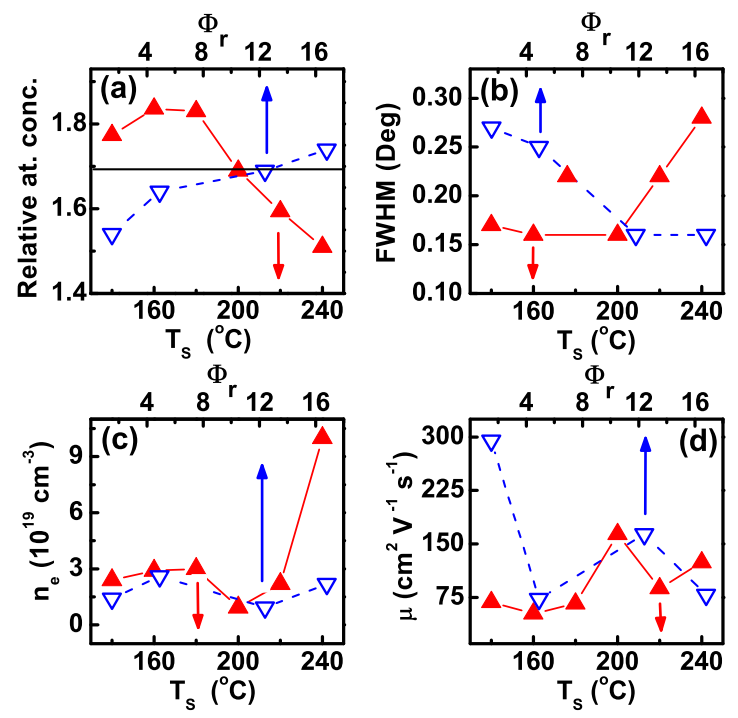

Figure 51: Electronic characterization of $\mathrm{Bi}_{2} \mathrm{Se}_{3}$ thin films. (a) Relative $\mathrm{Se} / \mathrm{Bi}$ atomic concentration determined by XPS, solid horizontal line is the measurement for a bulk $\mathrm{Bi}_{2} \mathrm{Se}_{3}$ single crystal; Uncertainty is $\pm 10 \%$; (b) FWHM for the (006) rocking curve; (c) carrier concentration; (d) carrier mobility. Lines are guides to the eye. Red solid upward triangles are dependencies on $T_{S}$ at $\Phi_{r}=11.8$, and blue open downward triangles are dependencies on $\Phi_{r}$ at $T_{S}=200{ }^{\circ} \mathrm{C}$.

(XPS) performed within less than 30 minutes after exposure to atmosphere to minimize oxidation. Separate tests showed that the oxidation effects appear in XPS spectra after several hours of atmospheric exposure (see section on flake oxidation). The relative atomic concentrations were calculated using the method in reference [10], and verified using a stoichiometric $\mathrm{Bi}_{2} \mathrm{Se}_{3}$ single crystal (please see section on $\mathrm{Bi}_{2} \mathrm{Se}_{3}$ flake oxidation) [95]. The measurement uncertainty of $10 \%$ was determined by repeating measurements on the same surface. Fig. 50 demonstrates typical XPS spectra for $\mathrm{Bi}_{2} \mathrm{Se}_{3}$. Both bismuth and selenium spectra exhibit doublet peaks, corresponding to the $4 \mathrm{f}_{7 / 2}, 4 \mathrm{f}_{5 / 2}, 3 \mathrm{~d}_{3 / 2}$, and $3 \mathrm{~d}_{5 / 2}$ energy states. The bismuth doublet peaks are distinct, while the selenium peaks have a significant overlap.

The dependence of relative atomic Se/Bi concentration $C_{r}$ on $T_{S}$ exhibited two regimes. At $T_{S}<180{ }^{\circ} \mathrm{C}, C_{r}$ was approximately constant with a slight excess of Se; it monotonically decreased at $T_{S}>180{ }^{\circ} \mathrm{C}$ (Fig. $51(\mathrm{a})$ ). At $\Phi_{r}=11.8$, the nominal stoichiometry was obtained at $T_{S} \approx 200{ }^{\circ} \mathrm{C}$, close to the temperature of the Se source. As expected, $C_{r}$ increased approximately linearly with increasing $\Phi_{r}$. 
A clear correlation was observed between the stoichiometry and the crystallinity of the film determined from the full width at half maximum (FWHM) of the (006) x-ray rocking curve (Fig. 51(b)). The instrumental resolution determined from substrate peak scans was $<0.14^{\circ}$. The set of films grown at a fixed $\Phi_{r}=11.8$ and varied $T_{S}<200{ }^{\circ} \mathrm{C}$ exhibited the smallest, almost resolution limited FWHM; the FWHM increased at $T_{S}>200{ }^{\circ} \mathrm{C}$. The increase of FWHM is correlated with the transition from excess Se to excess Bi concentration (Fig. 51(a)). The peak width decreased with increasing $\Phi_{r}$ at a fixed $T_{S}=200{ }^{\circ} \mathrm{C}$, stabilizing to a minimum constant value for $\Phi_{r}>12$, which is correlated with a crossover from excess $\mathrm{Bi}$ at $\Phi_{r}<12$ to excess Se at $\Phi_{r}>12$ (Fig. 51(a)). These behaviors indicate that the crystallinity of the films improves with increasing Se concentration regardless of which growth parameter is varied. Therefore, both $T_{S}$ and $\Phi_{r}$ can be adjusted to simultaneously optimize crystallinity and stoichiometry.

The most important parameters for potential applications of the TI films are carrier concentration $n_{e}$ and mobility $\mu_{e}$. These properties were determined by measurements of the Hall effect and conductivity in the van der Pauw geometry, as illustrated in Figs. 51(c,d) for $T=13$ K. Similar dependencies on the growth parameters were also observed at room temperature.

The dependence of $n_{e}$ on $T_{S}$ for $\Phi_{r}=11.8$ exhibits weak variations at low $T_{S}$, a minimum of $n_{e}=9.4 \times 10^{18} \mathrm{~cm}^{-3}$ at $T_{S}=200{ }^{\circ} \mathrm{C}$, and a dramatic increase for $T_{S}>200{ }^{\circ} \mathrm{C}$ (solid symbols in Fig. 51(c)). Since increasing $T_{S}$ results in a higher relative Bi concentration, one might expect a similar increase of n-type doping for small $\Phi_{r}$. However, the measured carrier concentration is almost independent of $\Phi_{r}$ at $T_{S}=200{ }^{\circ} \mathrm{C}$ (open symbols in Fig. 51(c)). Therefore, we attribute the increase of carrier concentration at high $T_{S}$ to desorption of Se from the surface due to its high vapor pressure. The resulting Se vacancies act as donors which do not become compensated by a possible increase of substitutional $\mathrm{Bi}_{\mathrm{Se}}$ acceptor defects.

In contrast to $n_{e}, \mu_{e}$ exhibited a relatively weak variation between 70 and $150 \mathrm{~cm}^{2} \mathrm{~V}^{-1} \mathrm{~s}^{-1}$ over nearly the entire range of growth parameters, with a dramatic increase to $\mu_{e} \approx 300 \mathrm{~cm}^{2} \mathrm{~V}^{-1} \mathrm{~s}^{-1}$ at small $\Phi_{r}=1.5$ and $T_{S}=200{ }^{\circ} \mathrm{C}$ (Fig. $51(\mathrm{c})$ ). For epitaxial $\mathrm{Bi}_{2} \mathrm{Se}_{3}$ grown on other substrates, $\mu_{e}$ ranges from 100 to $1000 \mathrm{~cm}^{2} \mathrm{~V}^{-1} \mathrm{~s}^{-1}[10,23,24,25]$. The slightly lower mobilities observed in our films may be associated with the high density of crystalline defects due to a lattice mismatch with the substrate. 

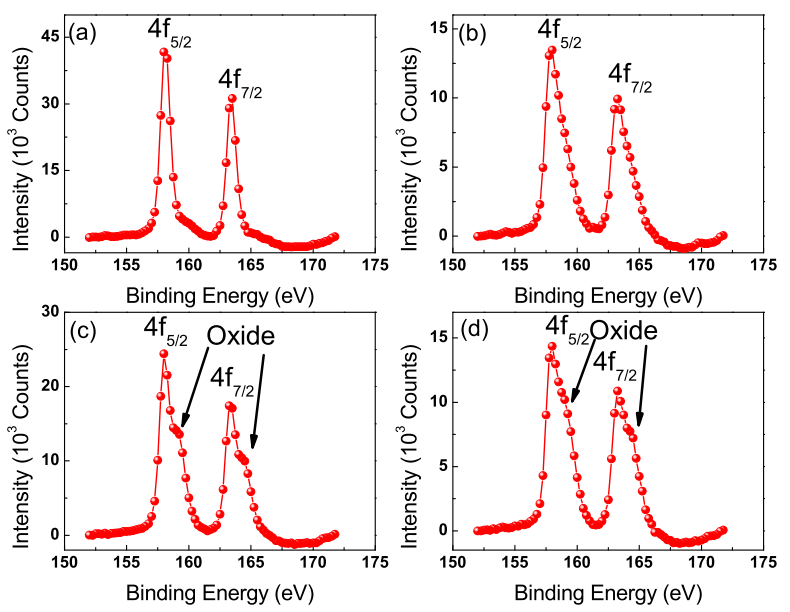

Figure 52: XPS spectra of bismuth $4 \mathrm{f}$ doublet peaks, for the exfoliated flake. The background has been subtracted. (a) Immediately following exfoliation. (b) Six hours of exposure. (c) Twelve hours of exposure. (d) Eighteen hours of exposure.

\section{$5.4 \quad \mathrm{Bi}_{2} \mathrm{Se}_{3}$ Flake Oxidation}

During the course of performing XPS measurements, it was discovered that samples that were exposed to atmosphere for extended periods of time presented anomalous peaks in their spectra. To understand how these peaks evolved over time, repeated measurements were performed on a stoichiometric flake after exposure to atmosphere. The bulk $\mathrm{Bi}_{2} \mathrm{Se}_{3}$ crystals were grown by combining stoichiometric quantities of elemental bismuth and selenium, $99.999 \%$ pure, in a quartz ampoule. The mixture was heated to $850^{\circ} \mathrm{C}$, and then cooled over twelve hours. The flakes were exfoliated by the scotch tape method, and the thickness was determined by averaging multiple measurements with a digital caliper.

Immediately following exfoliation, the flake was loaded into the XPS system and measured. The clean bismuth and selenium spectra can be seen in Figs. 52 (a) and 53 (a)respectively. Following measurement, the samples were removed from vacuum and left in air for six hours, after which they were inserted back into the system and measured again (Figs. 52 (b) and 53 (b)). After six hours of exposure, the spectra exhibit signs of oxidation. In the bismuth spectra, the oxide peaks overlap the main $4 \mathrm{f}_{7 / 2}$ and $4 \mathrm{f}_{5 / 2}$ spectral peaks and result in a slight broadening with respect to the unoxidized data. In selenium, a distinct oxide peak appears at higher energy. 

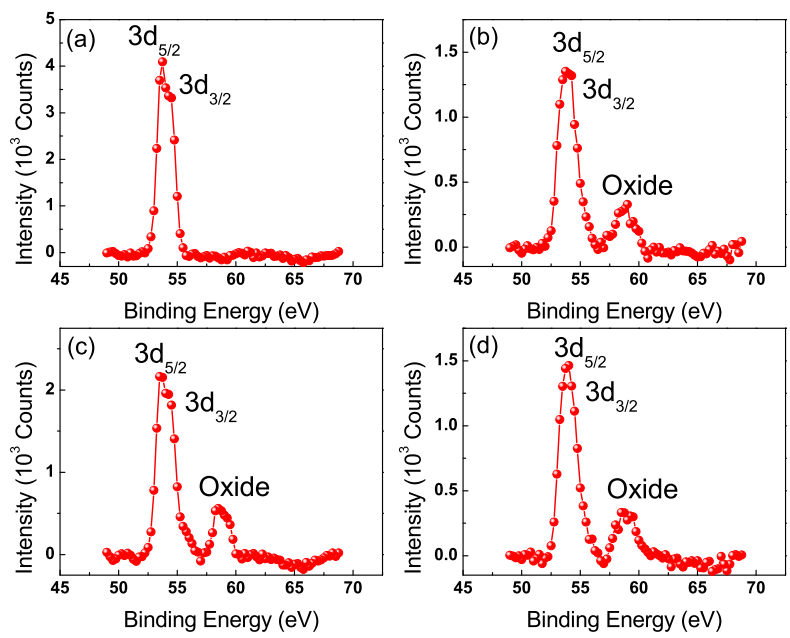

Figure 53: XPS spectra of selenium 3d doublet peaks, for the exfoliated flake. The background has been subtracted. (a) Immediately following exfoliation. (b) Six hours of exposure. (c) Twelve hours of exposure. (d) Eighteen hours of exposure.

Following six more hours of oxidation, the flake was measured again. At this point, the bismuth spectra begins to display shoulders, which indicate the emergence of distinct spectral peaks. Similarly for selenium, the separate oxide peak grows in area, indicating a higher degree of oxidation and divergence from nominal stoichiometry (Figs. 52 (c) and 53 (c)).

Finally, after eighteen hours of oxidation (Figs. 52 (d) and 53 (d)), the oxide peaks become more prominent. The bismuth spectra become wider, and the oxide peak in the selenium data also grows in total area.

\subsection{Conclusions}

In summary, we have demonstrated epitaxial growth of highly crystalline $\mathrm{Bi}_{2} \mathrm{Se}_{3}$ thin films on $\mathrm{Al}_{2} \mathrm{O}_{3}$ (110) substrates. Based on the analysis of the film morphology, stoichiometry, crystallinity, and carrier concentration, we infer the optimal film growth conditions that involve substrate temperature close to that of the Se source, and a high relative Se flux rate. These optimal conditions are likely determined by the balance between the favorable surface kinetics at higher temperatures and the desorption of Se from the film surface. The growth of high quality $\mathrm{Bi}_{2} \mathrm{Se}_{3}$ films on electrically 
insulating and optically transparent substrates enables electronic and optical studies of pristine interfacial states between the trivial insulator substrate and the TI film that are not affected by the free surface oxidation or the effects of surface roughness. 


\section{Bibliography}

[1] D. C. Ralph, and M.D. Stiles, J. Magn. Magn. Mater. 320, 2008 (.)

[2] S. Kaka, M.R. Pufall, W.H. Rippard, T.J. Silva, S.E. Russek, and J. A. Katine, Nature 437, 389 (2005).

[3] S.I. Kiselev, J.C. Sankey, I.N. Krivorotov, N.C. Emley, R.J. Schoelkopf, R.A. Buhrman, D.C. Ralph, Nature 425, 380 (2003).

[4] W.H. Meiklejohn and C.P. Bean, Phys. Rev. 102, 1413 (1956); Phys. Rev. 105, 904 (1957).

[5] J. C. Mallinson, Magneto-Resistive and Spin Valve Heads: Fundamentals and Applications, Elsivier, 2002.

[6] O. Ozatay, P.G. Gowtham, K.W. Tan, J.C. Read, K.A. Mkhoyan, M.G. Thomas, G.D. Fuchs, P.M. Braganca, E.M. Ryan, K.V. Thadani, J. Silcox, D.C. Ralph, and R.A. Buhrman, Nature Mat. 7, 567 (2008).

[7] M. Z. Hasan, and C. L. Kane, Rev. of Mod. Phys. 82, 3045 (2010).

[8] H. Zhang, C. X. Liu, X. -L. Qi, X. Dai, Z. Fang, and S. C. Zhang, Nat. Phys. 5, 438-442 (2009).

[9] P. Roushan, J. Seo, C. V. Parker, Y. S. Hor, D. Hsieh, D. Qian, A. Richardella, M. Z. Hasan, R. J. Cava and A. Yazdani, Nature 460, 1106 (2009).

[10] G. Zhang, H. Qin, J. Teng, J. Guo, Q. Guo, X. Dai, Z. Fang, and K. Wu, Appl. Phys. Lett. 95, 053114 (2009).

[11] L. Fu and C. L. Kane, Phys. Rev. Lett. 100, 096407 (2008). 
[12] C. Kittel, Phys. Rev. 73, 2 (1948).

[13] M.N. Baibich, J.M. Broto, A. Fert, N. Van Dau, F. Petroff, P. Etienne, G. Creuzet, A. Friedrich, and J. Chazelas, Phys. Rev. Lett. 61, 2472-2475 (1988).

[14] R. C. O'Handley, Modern Magnetic Materials, Wiley Inter Science, 2000

[15] J. Slonczewski, J. Magn. Magn. Mater. 159, L1 (1996).

[16] X.-L. Qi, and S.C. Zhang, Phys. Today. 63, 1 (33)2010.

[17] L. Fu, C. L. Kane, and E. J. Mele, Phys. Rev. Lett. 98, 106803 (2007).

[18] D. Hsieh, D. Qian, L. Wray, Y. Xia, Y. S. Hor, R. J. Cava and M. Z. Hasan, Nature 452, 970 (2008).

[19] D. Hsieh, Y. Xia, L. Wray, D. Qian, A. Pal, J. H. Dil, J. Osterwalder, F. Meier, G. Bihlmayer, C. L. Kane, Y. S. Hor, R. J. Cava and M. Z. Hasan, Science 323, 919 (2009).

[20] Y. Xia, D. Qian, D. Hsieh, L. Wray, A. Pal, H. Lin, A. Bansil, D. Grauer, Y.S. Hor, R.J. Cava and M.Z. Hasan, Nat. Phys. 5, 398 (2009).

[21] D. Hsieh, Y. Xia, D. Qian, L. Wray, J. H. Dil, F. Meier, J. Osterwalder, L. Patthey, J. G. Checkelsky, N. P. Ong, A. V. Fedorov, H. Lin, A. Bansil, D. Grauer, Y.S. Hor, R. J. Cava, and M. Z. Hasan, Nature 460, 1101 (2009).

[22] Y. S. Hor, A. Richardella, P. Roushan, Y. Xia, J. G. Checkelsky, A. Yazdani, M. Z. Hasan, N. P. Ong, and R. J. Cava, Phys. Rev. B 195208, 2008 (.)

[23] H. D. Li, Z. Y. Wang, X. Kan, X. Gui, H. T. He, Z. Wang, J. N. Wang, T. L. Wong, N. Wang, and M. H. Xie, New J. Phys. 12, 103038 (2010).

[24] A. Richardella, D. M. Zhang, J. S. Lee, A. Koser, D. W. Rench, A. L. Yeats, B. B. Buckley, D. D. Awschalom, and N. Samarth, Appl. Phys. Lett. 97, 262104 (2010).

[25] C. -L. Song, Y. -L. Wang, Y. -P Jiang, Y. Zhang, C. -Z. Chang, L. Wang, K. He, X. Chen, J. -F. Jia, Y. Wang, Z. Fang, X. Dai, X. -C. Xie, X. -L. Qi, S. -C. Zhang, Q. -K. Xue, and X. Ma, Appl. Phys. Lett. 97, 143118 (2010). 
[26] H. D. Li, Z. Y. Wang, X. Gui, T. L. Wong, N. Wang, and M. H. Xie, Appl. Phys. Lett. 98, $043104(2011)$.

[27] P.K. Karsen, and P.J. Dobson, eds. Reflection High Energy Electron Diffraction and Reflection Electron Imaging of Surfaces. Plenum, NATO ASI Ser. B., 1988.

[28] S.P. Svensson, P.O. Nilsson, and T.B. Andersson Phys. Rev. B 31, 5272 (1985).

[29] M.A. Herman, and H. Sitter Molecular Beam Epitaxy. Springer, Heidelberg, 1996.

[30] J. Heffernan, M. Kauer, K. Johnson, C. Zellweger, S.E. Hooper, and Bousquet, V. Phys. Stat. Sol. (a) 2005, 202 (868 874).

[31] P. Tabor, C. Keenan, S. Urazhdin, and D. Lederman, Appl. Phys. Lett. 99, 013111 (2011).

[32] M. Altissimo, Biomicrofluidics 4, 026503 (2010).

[33] A.T. Hindmarch, D.E. Parkes, and A.W. Rushfortha, Vacuum 86, 10 (2012).

[34] J. Thornton, Scanning Probe Microscopy Training Notebook. Veeco, 2000

[35] B.E. Warren, X-Ray Diffraction. Dover Publications, Mineola, 1990.

[36] N. W. Ashcroft, and N. D. Mermin, Solid State Physics. Thompson Learning, 1976.

[37] C. Giacovazzo, Fundamentals of Crystallography. Oxford Science Publications, New York, 2002.

[38] C. Keenan, Molecular Beam Epitaxy of Multiferroic YMnO $\mathrm{O}_{3}$ on c-plane GaN. Doctoral Dissertation, West Virginia University.

[39] E. Fullerton, I.K. Schuller, H. Vanderstraetan, and Y. Bruynseraede, Phys. Rev. B 45, 16 (1992)9292-9310.

[40] A. Gibaud, and S. Hazra, Current Science 78, 12 (2000)1467-1477.

[41] L. G. Parratt, Phys. Rev. 95, 1954 (359).

[42] L. Nvot, and P. Croce, Rev. Phys. Appl. 15, 1980 (761).

[43] P. J. Dobson, B. A. Joyce, J. H. Neave, and J. J. Zhang,Cryst. Growth 81, 1987 (1). 
[44] C.D.Wagner, W.M.Riggs, L.E.Davis, J.F.Moulder, and G.E.Mullenberg, Handbook of X-ray Photoelectron Spectroscopy, Perkin-Elmer Corp., 1979.

[45] S. Urazhdin and P. Tabor, J. Appl. Phys. 105, 066105 (2008).

[46] L. J. van der Pauw, Phillips. Tech. Rev. 20, 220-224 (8)1958.

[47] W.H. Rippard, M.R. Pufall, S.Kaka, S.E. Russek, and T.J. Silva, Phys. Rev. Lett. 92, 027201 (2004).

[48] L. Berger,Phys. Rev. B. 54, 9353 (1996).

[49] F.B. Mancoff, N.D. Rizzo, B.N. Engel, and S. Tehrani, Nature 437, 393 (2005).

[50] A. Ruotolo, V. Cros, B. Georges, A. Dussaux, J. Grollier, C. Deranlot, R. Guillemet, K. Bouzehouane, S. Fusil, and A. Fert, Nature. Nanotech. 4, 528-532 (2009).

[51] W.H. Rippard, M.R. Pufall, S.Kaka, T.J. Silva, S.E. Russek, and J.A. Katine, Phys. Rev. Lett. 95, $067203(2005)$.

[52] J.C. Sankey, P.M. Braganca, A.G.F. Garcia, I.N. Krivorotov, R.A. Buhrman, and D.C. Ralph, Phys. Rev. Lett. 96, 227601 (2006).

[53] B. Georges, J. Grollier, M. Darques, V. Cros, C. Deranlot, B. Macilhac, G. Faini, and A. Fert, Phys. Rev. Lett. 101, 017201 (2008).

[54] J.C. Sankey, I.N. Krivorotov, S.I. Kiselev, P.M. Braganca, N.C. Emley, R.A. Buhrman, and D.C. Ralph, Phys. Rev. B 72, 224427 (2005).

[55] V. S. Tiberkevich, A. N. Slavin, Joo-Von Kim, Phys. Rev. B 78, 092401 (2008).

[56] A. Pikovsky, M. Rosenbblum, and J. Kurths, Synchronization: a universal concept in nonlinear sciences, Cambridge, New York, 2001.

[57] R. Adler,Proc. IRE 34, 351 (1946).

[58] M. L. Schneider, A. B. Kos, and T. J. Silva, Appl. Phys. Lett. 86, 202503 (2005).

[59] A. Blaquiere, Nonlinear System Analysis, New York: Academic, 1966. 
[60] A. Slavin and V. Tiberkevich, IEEE Trans. Magn. 45, 1875 (2009).

[61] R. Bonin, G. Bertotti, C. Serpico, I.D. Mayergoyz, and M. d'Aquino, Eur. Phys. J. B 68, 221 (2009).

[62] F.B. Mancoff, R.W. Dave, N.D. Rizzo, T.C. Eschrich, B.N. Engel, and S. Tehrani, Appl. Phys. Lett. 83, 1596 (2003).

[63] N.C. Emley, I.N. Krivorotov, O. Ozatay, A.G.F. Garcia, J.C. Sankey, D.C. Ralph, and R.A. Buhrman, Phys. Rev. Lett. 96, 247204 (2006).

[64] S. Urazhdin and S. Button, Phys. Rev. B 78, 172403 (2008).

[65] S. Urazhdin, N.O. Birge, W.P. Pratt Jr., and J. Bass, Phys. Rev. Lett. 91, 146803 (2003).

[66] S. Urazhdin, N.O. Birge, W.P. Pratt Jr., and J. Bass, Appl. Phys. Lett. 84, 1516 (2004).

[67] L.P. Kadanoff, Statistical Physics: statics, dynamics and renormalization, World Scientific, 2000

[68] Z. Li, and S. Zhang, Phys. Rev. B 68, 024404 (2003).

[69] P.A.A. van der Heijden, T.F.M.M. Maas, W.J.M. de Jonge, J.C.S. Kools, F. Roozeboom, and P.J. can der Zaag, Appl. Phys. Lett. 72, 492 (1998).

[70] A. Scholl, M. Liberati, E. Arenholz, H. Ohldag, and J. Stohr, Phys. Rev. Lett 92, 247201 (2004).

[71] S. Urazhdin and N. Anthony Phys. Rev. Lett. 99, 46602 (2007).

[72] H. Ohldag, A. Scholl, F. Nolting, E. Arenhol, S. Maat, A.T. Young, M. Carey, and J. Stohr, Phys. Rev. Lett. 91, 017203 (2003).

[73] Uncompensated surfaces of real films become compensated once their finite roughness is taken into account.

[74] T.C. Schulthless and W.H. Butler, Phys. Rev. Lett. 81, 4516 (1998).

[75] A.P. Malozemoff, Phys. Rev. B 35, 3679 (1987); Phys. Rev. B 37, 7673 (1988).

[76] M.D. Stiles and R.D. McMichael, Phys. Rev. B 59, 3722 (1999). 
[77] K. Takano, R.H. Kodama, A.E. Berkowitz, W. Cao, and G. Thomas, Phys. Rev. Lett. 79, 1130 (1997).

[78] P. Miltenyi, M. Gierlings, J. Keller, B. Beschoten, G. Guntherodt, U. Nowak, and K.D. Usadel, Phys. Rev. Lett. 84, 4224 (2000).

[79] J.-I. Hong, T. Leo, D.J. Smith, A.E. Berkowitz, Phys. Rev. Lett. 96, 117204 (2006).

[80] R. Dieckmann,Z. Phys. Chem 107, 189 (1977).

[81] J. Nogues and I.K. Schuller, J. Magn. Magn. Mater. 192, 203 (1999).

[82] N.J. Gokemeijer, R.L. Penn, D.R. Veblen, and C.L. Chien, Phys. Rev B 63, 174422 (2001).

[83] M.D. Stiles and R.D. McMichael, Phys. Rev. B 60, 12950 (1999).

[84] S. Urazhdin and C.L. Chien, Phys. Rev. B 71, 220410 (2005).

[85] M.D. Stiles and R.D. McMichael, Phys. Rev. B 63, 064405 (2001).

[86] V.I. Nikitenko, V.S. Gornakov, L.M. Dedukh, Yu. P. Kabanov, A.F. Khapikov, A.J. Shapiro, R.D. Shull, A. Chaiken, and R.P. Michel, Phys. Rev. B 57, R8111 (1998).

[87] X. -L. Qi, T. L. Hughes, and S. -C. Zhang, Phys. Rev. B. 78, 195424 (2008).

[88] J. G. Analytis, J. -H. Chu, Y. Chen, F. Corredor, R. D. McDonald, Z. X. Shen, and I. R. Fisher,Phys. Rev. B 81, 205407 (2010).

[89] N. P. Butch, K. Kirshenbaum, P. Syers, A. B. Sushkov, G. S. Jenkins, H. D. Drew, and J. Paglione, Phys. Rev. B 81, 241301 (2010).

[90] M. König, S. Wiedmann, C. Brüne, A. Roth, J. Buhmann, L. W. Molenkamp, X. -L. Qi, and S. -C. Zheng, Science 318, 766 (2007).

[91] Y. L. Chen, J. G. Analytis, J. -H. Chu, Z. K. Liu, S. -K. Mo, X. L. Qi, H. J. Zhang, D. H. Lu, X. Dai, Z. Fang, S. -C. Zhang, I. R. Fisher, Z. Hussain, Z. -X. Shen, Science 325, 178 (2009).

[92] D. Hsieh, Y. Xia, D. Qian, L. Wray, F. Meier, J. H. Dil, J. Osterwalder, L. Patthey, A. V. Fedorov, H. Lin, A. Bansil, D. Grauer, Y. S. Hor, R. J. Cava and M. Z. Hasan, Phys. Rev. Lett. 103, $146401(2009)$. 
[93] W. Braun, Applied RHEED (Springer, Berlin, 1999), pp. 27-42.

[94] M. Björk and G. Andersson, J. Appl. Cryst 40, 1174 (2007).

[95] Crystal provided by Duck Young Chung from Argonne National Laboratory. 